\title{
Santé, cognition et capacité de travail après 50 ans: données internationales sur les marges extensive et intensive du travail
}

\author{
Vincent VANDENBERGHE*
}

\begin{abstract}
Résumé. L'auteur utilise l'Enquête sur la santé, le vieillissement et la retraite en Europe (SHARE) pour analyser le bien-fondé des réformes récentes visant à relever l'âge de la retraite. Il constate que la santé physique et les capacités cognitives se dégradent avec l'âge, ce qui abaisse les taux d'emploi des 50-54 ans. L'effet de la cognition est moins fort que celui de la santé. En outre, ces deux éléments influent plus sur l'emploi que sur les heures travaillées. Cependant, ils expliquent au plus 35 pour cent de la réduction du travail observée. La capacité de travail des seniors européens semble donc largement sous-utilisée.
\end{abstract}

Mots-clés: vieillissement, santé, cognition, capacité de travail, seniors, enquête SHARE.

\section{Introduction}

L'allongement de l'espérance de vie est sans doute la plus remarquable conséquence indirecte de la croissance économique et du progrès médical. Depuis la fin du XIX $\mathrm{X}^{\mathrm{e}}$ siècle, les populations des pays avancés gagnent 2,4 années de vie supplémentaires environ tous les dix ans (Oeppen et Vaupel, 2002). Toutefois,

* Institut de recherche économique et sociale, Louvain Institute of Data Analysis and Modelling in Economics and Statistics (IRES-LIDAM), Université catholique de Louvain (UCL); vincent. vandenberghe@uclouvain.be. L'auteur tient à remercier deux relecteurs anonymes pour leurs commentaires avisés et pour les améliorations apportées à des versions antérieures de l'article. Il souligne que les travaux dont il est rendu compte ont bénéficié d'un soutien financier dans le cadre du projet d'Action de recherche concertée (ARC) $n^{0}$ 18/23-088, et que des fonds ont aussi été mis à disposition pour la collecte des données de l'Enquête sur la santé, le vieillissement et la retraite en Europe (SHARE) utilisées pour les besoins de cet article (on trouvera le détail de ces financements à l'adresse http://www.share-project.org/organisation/funding.html).

Les articles paraissant dans la Revue internationale du Travail n'engagent que leurs auteurs, et leur publication ne signifie pas que le BIT souscrit aux opinions qui y sont exprimées.

Droits réservés (C) auteur(s), 2021.

Compilation et traduction des articles (C Organisation internationale du Travail, 2021. 
conjuguée à la baisse du taux de fécondité, cette évolution entraîne un vieillissement de la population et a d'importantes retombées économiques et sociopolitiques. Toutes choses égales par ailleurs, le vieillissement démographique va se traduire par une diminution de la population active et une hausse du taux de dépendance des personnes âgées. Ce phénomène risque de nuire à la croissance économique et à la qualité de vie en général s'il oblige les pouvoirs publics à consacrer moins de ressources publiques à l'éducation et aux infrastructures pour pouvoir financer les engagements envers les personnes âgées.

Pour lutter contre la contraction de la population active et la hausse du taux de dépendance, il est possible de jouer sur plusieurs paramètres, lesquels ont fait l'objet de travaux théoriques et empiriques (Acemoglu et Restrepo, 2018; Acemoglu, 2010; Vandenberghe, Waltenberg et Rigó, 2013). Ainsi, on peut envisager d'accroître le taux d'activité des femmes (du moins dans les pays où il reste faible), d'augmenter légèrement la durée du travail, de faire baisser le chômage, voire de réduire la durée de la formation initiale (Vandenberghe, 2020). Toutefois, jusqu'à présent, l'ajustement le plus souvent choisi par les responsables politiques consiste à relever l'âge effectif de la retraite. Les chercheurs de l'Organisation de coopération et de développement économiques (OCDE) ont fourni des données quantitatives qui montrent que l'indexation de l'âge de la retraite sur l'espérance de vie (de plus en plus longue) serait de nature à stabiliser les taux de dépendance aux alentours de leur niveau actuel et éviterait ainsi d'avoir soit à augmenter de manière spectaculaire les taux de prélèvement pour pouvoir financer les pensions par répartition, soit à imposer une baisse générale du montant des pensions (Oliveira Martins et al., 2005). De fait, les politiques de retraite plus restrictives mises en œuvre depuis le milieu des années 1990 ont fait progresser les taux d'emploi (Atalay et Barrett, 2015).

Toutefois, la question de l'équité de ces politiques est souvent posée parce que l'état de santé ou les capacités de certaines personnes pourraient être insuffisants pour leur permettre de continuer d'exercer une activité productive jusqu'à l'âge de la retraite. Il est donc important d'examiner dans quelle mesure les problèmes de santé ou la dégradation des capacités cognitives entravent la capacité des seniors à travailler.

Évaluer la capacité de travail (ou l'aptitude au travail) et son évolution avec l'âge n'est cependant pas chose aisée. Les données de l'Enquête sur la santé, le vieillissement et la retraite en Europe (Survey of Health, Ageing and Retirement in Europe ou SHARE) utilisées pour les besoins de cet article confirment l'existence d'une corrélation négative entre l'âge et la santé. Comme on pouvait s'y attendre, elles montrent également qu'en moyenne les individus travaillent beaucoup moins à mesure qu'ils vieillissent. Toutefois, même si ces deux constatations vont dans le même sens, cette convergence ne permet pas à elle seule de tirer des conclusions sur le lien entre âge et capacité de travail. En effet, l'exercice par les personnes âgées d'une activité rémunérée dépend de nombreux autres facteurs que la santé et la cognition. La retraite est l'un de ces facteurs. En vieillissant, les travailleurs deviennent éligibles à une pension de retraite et cessent généralement de travailler à partir de certains âges d'ouverture des 
droits ${ }^{1}$. Dans cet article, nous tentons donc d'évaluer l'influence de la santé et/ ou de la cognition sur la capacité de travail - laquelle pourrait rester nettement plus importante que ce que laissent penser les taux d'emploi des seniors. En nous concentrant sur la santé (et la cognition), nous ne cherchons pas à minimiser l'importance des autres facteurs qui exercent une influence sur l'emploi des seniors ni à tirer des conclusions sur le point de savoir s'il faut que les seniors travaillent - bon nombre de personnes préfèrent prendre leur retraite quels que soient leur état de santé et leurs performances cognitives, et d'autres, nombreuses également, peuvent ne pas pouvoir travailler en raison de l'insuffisance de la demande de main-d'œuvre âgée (Vandenberghe, 2013). Nous entendons simplement donner une idée de la quantité de travail que les seniors pourraient fournir compte tenu de leur état de santé ou de leurs capacités cognitives.

Pour estimer la capacité de travail, nous utilisons, en l'améliorant, une méthode initialement proposée par Cutler, Meara et Richards-Shubik (2013). Cette méthode, que nous dénommerons «méthode de Cutler» dans la suite de l'article, comporte deux étapes. La première consiste à estimer la relation entre emploi et santé entre 50 et 54 ans (en tenant compte d'autres caractéristiques, comme le niveau d'études et le sexe). Le choix de cette tranche d'âge permet de mesurer le lien entre santé et travail en l'absence de possibilité d'accès à des prestations de retraite (ou de préretraite), en d'autres termes avant que les travailleurs aient droit à des prestations de sécurité sociale ou autres prestations de remplacement du revenu. La deuxième étape consiste à utiliser les estimations issues de la première étape pour prédire la capacité de travail à un âge plus élevé (55-70 ans). L'idée est de combiner i) l'effet estimé de la santé (et d'autres caractéristiques) sur l'emploi parmi les personnes de 50 à 54 ans avec ii) l'état de santé réel des personnes de 55 à 70 ans. Cette approche postule que le lien entre santé et emploi est le même pour les deux groupes, mais que la capacité de travail diminue avec l'âge étant donné que l'état de santé se dégrade avec l'âge.

La deuxième étape permet d'obtenir une estimation contrefactuelle, qui peut être très différente du comportement réel des seniors à l'égard du travail. Elle permet de mesurer la contribution potentielle de la santé à l'évolution de l'aptitude au travail entre 55 et 70 ans. Contrairement à la plupart des travaux empiriques sur le vieillissement et sur l'emploi des seniors, sa principale fonction n'est pas de fournir une série de coefficients de régression décrivant le plus précisément possible la courbe âge/travail observée. Nous utilisons le travail tel qu'il ressort des données brutes comme un point de comparaison pour apprécier la capacité de travail «non utilisée», qui correspond à la différence entre l'estimation contrefactuelle (la capacité de travail prédite par l'état de santé) et le travail observé des seniors. Le plus important est d'obtenir la description la plus exacte possible de l'état de santé des personnes, afin de pouvoir calculer l'estimation contrefactuelle la plus plausible possible. La fiabilité des résultats dépend aussi largement de la manière dont la première étape de la méthode est mise en œuvre. L'objectif n'est en outre pas tant de maximiser le coefficient $\mathrm{R}^{2}$ du modèle estimé que de tenir compte des diverses dimensions de la santé

${ }^{1}$ Il y a relativement peu de temps encore, dans beaucoup de pays membres de l'OCDE, il était obligatoire de partir en retraite à 65 ans, voire à 60 ans. 
pouvant avoir une incidence sur l'emploi et de réduire le risque d'endogénéité. Les coefficients estimés par la méthode des moindres carrés ordinaires (MCO) peuvent être biaisés si des variables essentielles (par exemple les dimensions de la santé qui influent sur l'emploi) sont omises, en cas d'erreur de mesure ou en présence d'une variante particulière du biais de sélection connue sous le nom de «biais de justification» (Bound, 1991). Ce biais repose sur l'hypothèse que, pour justifier qu'ils ne travaillent pas, les répondants inactifs exagèrent leurs problèmes de santé comparativement aux répondants en activité.

La suite de l'article est organisée comme suit: dans la deuxième partie, nous présentons et examinons la littérature existante sur l'âge et la capacité de travail, ainsi que l'apport de notre étude. Dans la troisième partie, nous décrivons l'estimation en deux étapes de la capacité de travail, les principaux problèmes d'identification et leur traitement. La quatrième partie est consacrée à la description des microdonnées de SHARE sur le travail, la santé et la cognition utilisées dans l'article et la cinquième à la présentation détaillée des résultats. La sixième partie résume les principaux résultats de l'étude et propose plusieurs recommandations pour l'élaboration de politiques visant à relever les taux d'emploi des seniors.

\section{Lien entre âge et capacité de travail}

\subsection{Revue de la littérature}

L’article porte sur la capacité de travail et son évolution avec l'âge. Il suit Blundell et al. (2017) pour la méthodologie économétrique et Wise (2017) pour la dimension internationale. Il représente une contribution aux travaux de recherche sur le vieillissement et le travail, en particulier sur les obstacles à l'emploi des seniors, et accorde une importance relative plus grande au versant offre qu'au versant demande du marché du travail. Nous employons l'adjectif "relative» parce que, s'agissant du travail, nos observations se rapportent systématiquement aux interactions entre l'offre et la demande de main-d'œuvre. Plus précisément, nous examinons ici dans quelle mesure la dégradation de la santé et/ ou des capacités cognitives limite la capacité de travail des personnes. Il existe à l'évidence de nombreux autres obstacles à l'emploi des seniors, mais nous ne les examinerons pas parce que ce n'est pas nécessaire pour évaluer la capacité de travail. Certains de ces obstacles sont liés au versant demande du marché du travail - ils sont dus à la réticence de certaines entreprises à employer ou recruter des seniors, par exemple - et ont été étudiés par Hutchens (1986 et 2010), Dorn et Sousa-Poza (2010), Dostie (2011), Skirbekk (2004), van Ours et Stoeldraijer (2011), Vandenberghe, Waltenberg et Rigó (2013), et Delmez et Vandenberghe (2018). D'autres sont en rapport avec l'offre de travail, mais doivent être distingués des obstacles liés à l'état de santé et à la cognition étudiés ici. Les économistes ont décrit le rôle important que jouent les régimes de retraite (et de préretraite) et autres dispositifs de protection sociale dans les sorties précoces du marché du travail (Blöndal et Scarpetta, 1999; Jousten et al., 2010). À titre d'exemple, il existe une abondante littérature économique sur le départ en retraite conjoint, c'està-dire sur la propension à coordonner les décisions de départ au sein des couples dont les deux membres travaillent (voir Michaud, Van Soest et Bissonnette, 2020). 
Globalement, la capacité de travail des seniors semble s’être améliorée au fil du temps. Oeppen et Vaupel (2002) utilisent des données recueillies au niveau des pays et constatent que, depuis cent soixante ans, «l'espérance de vie maximale» ${ }^{2}$ augmente chaque année de manière constante de trois mois environ par an. D’autres chercheurs se sont appuyés sur la Global Burden of Disease Study ${ }^{3}$ pour montrer que le taux de morbidité ${ }^{4}$ avait diminué au fil du temps et dans toutes les régions du monde entre 1990 et 2017 (Chang et al., 2019). Au début des années 2010, les économistes américains Cutler, Ghosh et Landrum (2013) ont mis en évidence une diminution de la morbidité dans la dernière partie de la vie, ce qui expliquerait pourquoi des auteurs tels que Börsch-Supan (2014) remettent en cause l'idée selon laquelle la plupart des travailleurs sont en trop mauvaise santé pour continuer de travailler jusqu’à 70 ans. Dans notre article, nous testons également cette idée en nous appuyant sur des données internationales recueillies au niveau individuel pour évaluer la capacité de travail au-delà de 50 ans. Ce faisant, nous enrichissons la littérature consacrée à la capacité de travail, qui comprend notamment les travaux récents de Jousten et al. (2010), Coile, Milligan et Wise (2016), Banks, Emmerson et Tetlow (2016) et l'étude coordonnée par Wise (2017), qui, comme la nôtre, a une importante dimension internationale.

Dans cet article comme dans les travaux précités, la capacité de travail est définie comme le niveau de travail attendu si l'on suppose que les problèmes de santé (ou les problèmes cognitifs) sont les seuls déterminants de la propension à travailler des seniors. Comme indiqué plus haut, nous l'estimons au moyen de la méthode en deux étapes définie par Cutler. Nous avons écarté l'autre méthode possible (Milligan et Wise, 2012; De Souza, Queiroz et Skirbekk, 2019) parce qu'elle fait appel à une définition de la santé beaucoup moins précise, reposant sur le taux de mortalité à un âge donné.

\subsection{Contribution de notre article aux travaux sur la capacité de travail}

Contrairement à de nombreuses études existantes utilisant la méthode de Cutler, notre article a une forte dimension internationale, puisqu'il mesure la capacité de travail dans vingt pays simultanément. De plus, comparativement à l'étude coordonnée par Wise (2017), citée plus haut, il présente l'intérêt d'utiliser les données de l'enquête SHARE, qui permet d'obtenir une série unique de données totalement harmonisées (Börsch-Supan et al., 2013).

Pour que la méthode de Cutler soit fiable, il faut utiliser des microdonnées à granularité fine, décrivant correctement l'état de santé et son évolution avec l'âge. Cette condition est particulièrement importante pour la deuxième étape de la méthode. De ce point de vue, la série de données issues de SHARE comprend de nombreux indicateurs de santé et de cognition susceptibles d’avoir un impact

\footnotetext{
${ }^{2}$ Espérance de vie la plus longue observée au cours d'une année donnée.

${ }^{3}$ Une étude épidémiologique observationnelle internationale conduite par l'Institute for Health Metrics and Evaluation de l'Université de Washington (https://www.thelancet.com/gbd).

4 Taux de morbidité par âge standardisés sur l'âge.
} 
sur la capacité de travail. Parmi ces indicateurs figurent des évaluations autodéclarées ou subjectives de l'état de santé physique, mais aussi de nombreuses pathologies précises diagnostiquées par un médecin - diabète, hypertension et arthrose, par exemple -, de même que des problèmes médicaux mesurables, comme la limitation de la mobilité et la force de préhension.

Autre point fort de notre article: il porte non seulement sur les problèmes de santé physiques, mais aussi sur la cognition, qui contribue sans doute de plus en plus à la capacité de continuer d'exercer une activité professionnelle puisque les travaux physiques ont aujourd'hui un poids plus faible dans l'économie. Les données de SHARE sont relativement riches en items permettant d'obtenir des scores à des tests de mémorisation, de mathématiques et de calcul, scores qui peuvent être utilisés pour calculer un indicateur de performance cognitive. À notre connaissance, la cognition n'est pas mesurée dans les autres travaux de recherche qui font appel à la méthode de Cutler et a suscité moins d'intérêt dans les études économiques portant de manière plus générale sur les déterminants de l'activité professionnelle des seniors. L'étude de Blundell et ses coauteurs (2017) sur le Royaume-Uni de Grande-Bretagne et d'Irlande du Nord et les ÉtatsUnis d’Amérique («Royaume-Uni» et «États-Unis» dans la suite du texte) constitue cependant une exception notable. D’autres auteurs ont examiné la relation entre cognition et revenu, mais ils ne se sont pas intéressés au lien entre cognition et travail (Anger et Heineck, 2010). La cognition en tant que dimension du vieillissement a en revanche fait l'objet de nombreux travaux non économiques, plus précisément dans le domaine de la psychologie cognitive (Salthouse, 2010) et dans des disciplines connexes.

Par ailleurs, notre article examine à la fois la marge extensive du travail ('emploi) et la marge intensive (les heures travaillées), alors que la plupart des travaux existants ne portent que sur la décision de travailler et n'examinent pas le lien entre l'altération de la santé et/ou de la cognition et la durée du travail 5 .

La méthode de Cutler peut être appliquée facilement au moyen de calculs économétriques simples. Elle a souvent été utilisée ainsi, y compris dans des comparaisons internationales (Wise, 2017). Sa fiabilité dépend cependant en grande partie de la manière dont la première étape est mise en œuvre. Dans la plupart des applications existantes, l'emploi est régressé sur l'état de santé autodéclaré par la méthode des MCO. Le coefficient obtenu est ensuite utilisé lors de la deuxième étape pour estimer les prédictions de la capacité de travail. Blundell et ses coauteurs (2017) rappellent cependant que la réalisation d'une estimation correcte de l'effet de la santé sur le travail constitue à elle seule un programme de recherche. Plus précisément, Bound (1991) pose la question de l'ampleur du «biais de justification» par rapport à l'erreur de mesure habituelle de l'état de santé autodéclaré. Pour justifier leur inactivité, les répondants qui ne travaillent pas peuvent surestimer l'obstacle au travail que représente un problème de santé par rapport à ce que déclarerait un répondant qui travaille,

\footnotetext{
${ }^{5}$ Une enquête relativement récente de l'OCDE et de l'Union européenne (2016) ne mentionne que les études de Pelkowski et Berger (2004) et de Moran, Farley Short et Hollenbeak (2011), qui parviennent l'une et l'autre à la conclusion que cette altération a un impact négatif sur la durée du travail.
} 
ce qui engendre un biais vers le haut des coefficients estimés par les MCO. Dans cet article, nous tentons de corriger ce biais en ayant recours à une régression par variables instrumentales, à savoir que nous instrumentons la santé subjective par des indicateurs de santé plus «objectifs».

\section{Méthode}

\subsection{Présentation générale}

La première étape de la méthode de Cutler consiste à estimer la relation entre la santé et/ou la cognition d'une part et la propension à travailler d'autre part. Les études existantes assimilent le travail à l'emploi ( $E M P L)$, ce qui signifie qu'elles portent sur la marge extensive du travail. Dans cet article, nous nous intéressons aussi à la marge intensive, autrement dit au nombre d'heures travaillées (HOURS), et à ce que nous dénommons le «travail total», soit le nombre d'heures de travail au sein de la population cible totale, associant ainsi marge extensive et marge intensive.

L'échantillon utilisé dans la première étape est composé de personnes relativement jeunes. Nous avons opté pour la tranche d'âge 50-54 ans pour les raisons exposées plus haut (survenue relativement fréquente de problèmes de santé/ de cognition, mais faible probabilité que les décisions à l'égard du travail soient motivées par la possibilité de percevoir des prestations de retraite ou de préretraite). Algébriquement, nous régressons - pour chacun des pays représentés dans la série de données issue de SHARE - les différentes dimensions du travail (EMPL, HOURS et WORK) sur la santé et/ou la cognition, comme ceci:

$Z_{i}^{50-54}=\beta_{0}^{z}+\beta_{h}^{z} H E A L T H_{i}^{50-54}+\beta_{c}^{z} C O G N_{i}^{50-54}+\gamma_{i}^{z} X_{i}^{50-54}+\varepsilon_{i}^{z}$,

étant entendu que $Z=E M P L, H O U R S, W O R K$.

C'est durant cette étape qu'interviennent les problèmes d'identification économétrique décrits dans la partie 2.2. L'essentiel à ce stade est toutefois de relever que le vecteur des coefficients $\widehat{\beta_{0}^{z}}, \widehat{\beta_{h}^{z}}$ et $\widehat{\beta_{c}^{\mathrm{z}}}$ est obtenu par estimation de l'équation (1). À noter également que le modèle contient des variables de contrôle $X_{i}^{50-54}$ (niveau d'études, sexe et effets fixes dans le temps, variables correspondant au coefficient $\widehat{\gamma}$ ). Lors de la deuxième étape, $\widehat{\beta_{0}^{z}}, \widehat{\beta_{h}^{z}}$ et $\widehat{\beta_{c}^{z}}$ sont appliqués à la santé, à la cognition et aux variables de contrôle caractérisant les individus d'âge $a=50, \ldots, 70$, et décrivant l'évolution de la santé ou de la cognition avec l'âge, informant ainsi de la capacité de travail attendue des répondants:

$\widehat{Z}_{i}^{a}=\widehat{\beta_{0}^{\mathrm{z}}}+\widehat{\beta_{h}^{\mathrm{z}}} \mathrm{HEALTH}_{i}^{a}+\widehat{\beta_{c}^{\mathrm{z}}} \operatorname{COGN}_{i}^{a}$,

avec $Z=E M P L, H O U R S$, WORK et $a=50, \ldots, 55, \ldots, 70$.

Les valeurs attendues dans l'équation (2) peuvent alors être utilisées pour le calcul de divers indicateurs synthétiques de la diminution de la capacité de travail en termes absolus comme en termes relatifs. Nous nous concentrerons sur l'impact cumulé de l'âge durant vingt années, en comparant les résultats obtenus pour les personnes de 50 ans avec ceux obtenus pour les personnes de 70 ans. Pour quantifier la capacité de travail inutilisée, nous comparons les valeurs de la capacité de travail (moyenne) prédites et les valeurs observées. Selon la même logique, nous calculons le pourcentage $\delta$ correspondant à la 
proportion de la variation du travail réel/observé entre 50 et 70 ans qui peut être imputée à la diminution de la capacité de travail (et par conséquent de la santé ou de la cognition):

$\delta^{Z, 50-70}=\frac{\widehat{Z}^{70}-\widehat{Z}^{50}}{Z^{70}-Z^{50}}$

Dans cette équation, $\delta$ peut être calculé comme le rapport de deux coefficients de variables muettes à l'âge de 70 ans $\left(\theta^{70}, \pi^{70}\right)$, obtenus (respectivement) en régressant le travail prédit ou le travail observé sur des variables muettes d'âge (50 étant l'âge de référence, correspondant aux constantes), ce qui donne

$\hat{Z}_{i}^{a}=\theta^{50}+\theta^{51} A G E_{i}^{51}+\ldots+\theta^{70} A G E_{i}^{70}+v_{i}^{z}$, et

$Z_{i}^{a}=\pi^{50}+\pi^{51} A G E_{i}^{51}+\ldots+\pi^{70} A G E_{i}^{70}+\mu_{i}^{z}$,

où $A G E_{i}^{51}$ prend la valeur 1 si $a=51$, et la valeur 0 dans les autres cas; de même, $A G E_{i}^{70}$ prend la valeur 1 si $a=70$, et la valeur 0 dans les autres cas; et $Z=E M P L$, HOURS, WORK:

$\delta^{Z, 50-70}=\frac{\hat{\theta}^{70}}{\widehat{\pi}^{70}}$.

\subsection{Identifier de manière fiable le lien entre santé ou cognition et travail}

L'estimation de la relation entre santé ou cognition et travail parmi les répondants âgés de 50 à 54 ans revêt une importance capitale dans la méthodologie exposée ci-dessus. Blundell et ses coauteurs (2017) présentent un excellent recensement des biais possibles. Nous nous concentrons ici sur ceux qui concernent les plus utilisées des estimations par les MCO basées sur des réponses subjectives à des questions sur l'état de santé.

Jusqu'à présent, de manière générale, la littérature sur la santé et le travail (voir Baker, Stabile et Deri, 2004) interprète les indicateurs subjectifs comme des mesures entachées de «bruits» du stock de santé latent (inobservé) $H^{6}$. Si HEALTH dans l'équation (1) représente l'indicateur de la santé subjectif construit au moyen des items sur l'état de santé subjectif/autodéclaré7, il peut être différent du stock de santé réel:

HEALTH $_{i}=H_{i}+\tau_{i}$.

Le terme de déviation $\tau_{i}$ correspond alors à une erreur de mesure/de déclaration (distribuée de manière aléatoire) qui induit un biais d'atténuation. Le terme $\operatorname{VAR}(\tau)$ qui figure dans le dénominateur de l'équation (7) rend compte de ce biais d'atténuation, le bruit (autrement dit un terme $\operatorname{VAR}(\tau)$ plus élevé) tirant vers zéro le coefficient $\beta^{Z}$ estimé par les MCO,

$\beta^{Z}=\frac{\tilde{\beta}^{Z} \operatorname{VAR}(H)}{\operatorname{VAR}(H)+\operatorname{VAR}(\tau)}$.

Il est toutefois peu probable que $\tau_{i}$ ne corresponde qu'à un «bruit». En pareil cas, l'équation (7) devient

$\beta^{Z}=\frac{\tilde{\beta}^{Z} \operatorname{VAR}(H)+\operatorname{COV}(\varepsilon, \tau)}{\operatorname{VAR}(H)+\operatorname{VAR}(\tau)}$,

${ }^{6}$ Ce stock peut être considéré comme le «véritable» indicateur de la santé influençant le travail.

${ }^{7}$ Voir la dernière colonne du tableau 2. 
et le terme $\operatorname{COV}(\varepsilon, \tau)$ du numérateur - où $\varepsilon$ est le résidu de l'équation (1) estimée dans la première étape - est différent de zéro. De plus, il est probable qu'un $\operatorname{COV}(\varepsilon, \tau)$ supérieur à 0 soit dû à un biais de justification (Baker, Stabile et Deri, 2004). Ce biais se produit lorsque les valeurs de $H$ (donc de $\tau$ ) déclarées s'expliquent par la situation professionnelle des répondants, à savoir que les individus qui ne travaillent pas déclarent se trouver dans un état de santé relativement mauvais afin de justifier leur inactivité. Comme l'indique l'équation (8), il peut se traduire par un coefficient $\beta^{Z}$ estimé par les MCO supérieur au coefficient d'intérêt $\widetilde{\beta}^{Z}$.

Les études réalisées jusqu'à présent ne permettent pas de tirer des conclusions tranchées concernant le poids relatif de ces deux biais. L'étude d'O'Donnell, Van Doorslaer et Van Ourti (2015) laisse penser que le biais de justification l'emporte, ce qui signifie que le coefficient $\beta^{Z}$ est biaisé vers le haut. En revanche, Stern (1989) et Dwyer et Mitchell (1999) ne montrent pas que le biais de justification est supérieur au biais d'atténuation. C'est pourquoi nous utilisons ici des variables instrumentales pour construire ce qui sera notre modèle économétrique préféré. Les tests physiques ou de dextérité auxquels sont soumises les personnes interrogées dans le cadre de l'enquête SHARE offrent un large choix de variables instrumentales potentielles (tableau 3$)^{8}$. Il est possible de montrer simplement que tout sous-ensemble d'indicateurs «objectifs» de l'état de santé peut être utilisé pour obtenir des estimations non biaisées de $\tilde{\beta}^{Z}$. La première étape du modèle à variables instrumentales (IV, pour instrumental variables, dans les équations et les tableaux) consiste à régresser l'indicateur de santé subjectif $(H E A L T H)$ sur l'indicateur objectif $\left(H^{O}\right)$. L'équation s'écrit ainsi:

$\hat{\eta}=\frac{\operatorname{COV}\left(H E A L T H, H^{O}\right)}{\operatorname{VAR}\left(H^{O}\right)}$,

où $H E A L T H=H+\tau$.

$\mathrm{Si}$ - comme ce doit être le cas pour tout instrument fiable $-\operatorname{COV}\left(H^{O}, \tau\right)$ est égal à 0 , et en supposant des variances standardisées, ce coefficient issu de la première étape devient:

$\hat{\eta}=\frac{\operatorname{COV}\left(H, H^{O}\right)}{\operatorname{VAR}(H)}$

Dans la deuxième étape, la situation des répondants sur le marché du travail $(Z)$ est régressée sur la valeur prédite par $\hat{\eta} H^{O}$ :

$\hat{\beta}^{Z, I V}=\frac{\operatorname{COV}\left(Z, \hat{\eta} H^{O}\right)}{\hat{\eta}^{2} \operatorname{VAR}\left(H^{O}\right)}$,

$\hat{\beta}^{Z, I V}=\tilde{\beta}^{Z} \frac{\operatorname{COV}\left(H, H^{O}\right)}{\hat{\eta} \operatorname{VAR}(H)}=\tilde{\beta}^{Z}$.

8 Tous les indicateurs de santé «objectifs» comportent des éléments résiduels subjectifs ou endogènes («Un médecin vous a-t-il déjà dit que vous aviez du diabète?»; les limitations ne sont pas mesurées par l'enquêteur: elles sont citées par la personne interrogée; la force de préhension est mesurée par l'enquêteur mais suppose la coopération de la personne interrogée). En conséquence, l'utilisation des indicateurs issus de l'enquête SHARE (tableau 3) ne devrait pas être considérée comme permettant de résoudre parfaitement le problème de l'erreur de mesure et du biais de justification. 
Tableau 1. Observations par pays et par vague

\begin{tabular}{|c|c|c|c|c|c|c|c|}
\hline \multirow[t]{2}{*}{ Pays } & \multicolumn{6}{|l|}{ Vague } & \multirow[t]{2}{*}{ Total } \\
\hline & 1 & 2 & 4 & 5 & 6 & 7 & \\
\hline Allemagne & 2926 & 2585 & 1610 & 5616 & 4354 & 3797 & 20888 \\
\hline Autriche & 1522 & 1181 & 5096 & 4303 & 3362 & 3187 & 18651 \\
\hline Belgique & 3637 & 3147 & 5194 & 5546 & 5716 & 4853 & 28093 \\
\hline Croatie & - & - & - & - & 2447 & 2379 & 4826 \\
\hline Danemark & 1614 & 2551 & 2236 & 4064 & 3668 & 3216 & 17349 \\
\hline Espagne & 2276 & 2375 & 3663 & 6579 & 5569 & 4676 & 25138 \\
\hline Estonie & - & - & 6757 & 5699 & 5559 & 5070 & 23085 \\
\hline France & 2965 & 2900 & 5666 & 4432 & 3887 & 3298 & 23148 \\
\hline Grèce & 2666 & 3236 & - & - & 4821 & 3039 & 13762 \\
\hline Hongrie & - & - & 2999 & - & - & 1531 & 4530 \\
\hline Irlande & - & 1007 & - & - & - & - & 1007 \\
\hline Israël & 2296 & 2389 & - & 2567 & 2015 & 2122 & 11389 \\
\hline Italie & 2505 & 2929 & 3507 & 4664 & 5219 & 4529 & 23353 \\
\hline Luxembourg & - & - & - & 1591 & 1548 & 1240 & 4379 \\
\hline Pologne & - & 2429 & 1721 & - & 1807 & 4651 & 10608 \\
\hline Portugal & - & - & 1962 & - & 1665 & 487 & 4114 \\
\hline Slovénie & - & - & 2708 & 2924 & 4197 & 3681 & 13510 \\
\hline Suède & 2996 & 2765 & 1963 & 4516 & 3884 & 3183 & 19307 \\
\hline Suisse & 952 & 1460 & 3680 & 3000 & 2775 & 2386 & 14253 \\
\hline Tchéquie & - & 2669 & 5392 & 5541 & 4801 & 4192 & 22595 \\
\hline Total & 26355 & 33623 & 54154 & 61042 & 67294 & 61517 & 303985 \\
\hline
\end{tabular}

Note: Observations indiquées pour les vagues 1 (2004), 2 (2007), 4 (2011), 5 (2013), 6 (2015) et 7 (2017). La vague 3 (2009), qui ne contient que des histoires de vie, n'est pas utilisée ici. Le nombre total d'observations est de 303985.

Source: SHARE 2004-2017.

\section{Données}

Dans cet article, nous utilisons les vagues 1, 2 et 4 à 7 de l'enquête SHARE ${ }^{9}$, ce qui nous permet d'obtenir au total 303985 observations (tableau 1). Tous les répondants ont au moins 50 ans lorsqu'ils sont interrogés. En raison de diverses limites liées aux données (valeurs manquantes, absence d’observations répétées pour les pays qui n’ont participé qu’à une seule vague), vingt des vingt-neuf pays participants ont été inclus dans l'analyse (Allemagne, Autriche, Belgique, Croatie, Danemark, Espagne, Estonie, France, Grèce, Hongrie, Irlande, Israël, Italie, Luxembourg, Pologne, Portugal, Slovénie, Suède, Suisse et Tchéquie). SHARE contient de nombreux items décrivant le travail des individus (situation professionnelle et durée du travail pour les salariés), mais aussi leur état de santé et leurs capacités cognitives. Nous répartissons les variables de santé dans deux grandes catégories: les variables «subjectives» (tableau 2) et les variables «objectives» (tableau 3). Comme indiqué dans le tableau 2, la plupart des items de la série de données issues de SHARE sont autodéclarés ou subjectifs, mais

\footnotetext{
${ }^{9}$ La vague 3, qui ne concernait que des histoires de vie, n'est pas utile ici.
} 
Tableau 2. Santé physique subjective (tous âges groupés)

\begin{tabular}{|c|c|c|c|c|c|c|c|}
\hline Pays & $\begin{array}{l}\text { Altération de } \\
\text { l'état général }\end{array}$ & $\begin{array}{l}\text { Mauvaise } \\
\text { santé perçue } \\
\text { (échelle } \\
\text { États-Unis) }\end{array}$ & $\begin{array}{l}\text { Maladie } \\
\text { de longue } \\
\text { durée }\end{array}$ & $\begin{array}{l}\text { Limitation } \\
\text { des activités }\end{array}$ & $\begin{array}{l}\text { Nombre de } \\
\text { limitations } \\
\text { (activités } \\
\text { quotidiennes, } \\
\text { ADL) }\end{array}$ & $\begin{array}{l}\text { Nombre } \\
\text { de limitations } \\
\text { (instrumentales, } \\
\text { IADL) }\end{array}$ & $\begin{array}{l}\text { Indicateur } \\
\text { de santé } \\
\text { subjectif }\end{array}$ \\
\hline Allemagne & 3,26 & 3,26 & 2,53 & 2,29 & 0,24 & 0,35 & 0,06 \\
\hline Autriche & 3,02 & 3,02 & 3,01 & 2,35 & 0,25 & 0,50 & $-0,09$ \\
\hline Belgique & 3,01 & 3,01 & 3,08 & 2,36 & 0,30 & 0,51 & $-0,10$ \\
\hline Croatie & 3,34 & 3,34 & 2,61 & 2,29 & 0,25 & 0,48 & 0,11 \\
\hline Danemark & 2,57 & 2,57 & 2,94 & 2,51 & 0,17 & 0,34 & $-0,39$ \\
\hline Espagne & 3,38 & 3,38 & 2,96 & 2,51 & 0,39 & 0,73 & 0,09 \\
\hline Estonie & 3,87 & 3,87 & 2,07 & 2,11 & 0,36 & 0,63 & 0,51 \\
\hline France & 3,22 & 3,22 & 3,11 & 2,39 & 0,25 & 0,43 & $-0,03$ \\
\hline Grèce & 2,99 & 2,99 & 3,57 & 2,64 & 0,17 & 0,46 & $-0,27$ \\
\hline Hongrie & 3,67 & 3,67 & 2,28 & 2,25 & 0,28 & 0,63 & 0,33 \\
\hline Irlande & 2,56 & 2,56 & 3,46 & 2,62 & 0,22 & 0,33 & $-0,47$ \\
\hline Israël & 3,21 & 3,21 & 2,78 & 2,39 & 0,45 & 0,98 & 0,11 \\
\hline Italie & 3,27 & 3,27 & 3,35 & 2,44 & 0,28 & 0,50 & $-0,04$ \\
\hline Luxembourg & 3,08 & 3,08 & 3,05 & 2,36 & 0,22 & 0,40 & $-0,09$ \\
\hline Pologne & 3,69 & 3,69 & 2,33 & 2,18 & 0,40 & 0,63 & 0,38 \\
\hline Portugal & 3,73 & 3,73 & 2,85 & 2,24 & 0,44 & 0,63 & 0,34 \\
\hline Slovénie & 3,34 & 3,34 & 2,99 & 2,33 & 0,25 & 0,49 & 0,06 \\
\hline Suède & 2,78 & 2,78 & 2,83 & 2,43 & 0,18 & 0,32 & $-0,25$ \\
\hline Suisse & 2,71 & 2,71 & 3,58 & 2,58 & 0,10 & 0,19 & $-0,44$ \\
\hline Tchéquie & 3,36 & 3,36 & 2,78 & 2,25 & 0,26 & 0,47 & 0,12 \\
\hline \multicolumn{8}{|c|}{$\begin{array}{l}\text { Note: État de santé général (échelle européenne) = } 1 \text { (bon)/5 (mauvais); santé perçue (échelle utilisée aux États-Unis) }=1 \\
\text { (bonne)/5 (mauvaise); maladie de longue durée = oui }(1) / \mathrm{non}(0) ; \text { limité(e) dans les activités quotidiennes }=3 \text { (non)/1 (grave- } \\
\text { ment); nombre de limitations des activités quotidiennes }(\mathrm{ADL})=\text { échelle graduée de } 0 \text { à } 6 ; \text { nombre de limitations des activi- } \\
\text { tés quotidiennes instrumentales (IADL) = échelle graduée de } 0 \text { à } 9 \text {. Pour calculer l'indicateur de santé subjectif, on retient la } \\
\text { première composante principale de chaque variable (plus elle est élevée, plus la santé perçue est mauvaise). L'analyse en } \\
\text { composantes principales est réalisée sur pays groupés. Les valeurs indiquées correspondent aux valeurs prédites des scores } \\
\text { divisées par l'écart type. }\end{array}$} \\
\hline
\end{tabular}

beaucoup renvoient à des diagnostics posés par des professionnels de santé (infarctus du myocarde, hypertension, hypercholestérolémie, accident vasculaire cérébral, diabète, pneumopathie, cancer) ou sont mesurés par les enquêteurs - c'est par exemple le cas de la limitation de la mobilité et de la force de préhension maximale (voir les deux dernières colonnes du tableau 3). Les items utilisés pour évaluer les capacités cognitives sont répertoriés dans le tableau 4. Ils consistent en des scores obtenus à des tests de mémorisation ou de calcul/ mathématiques.

À partir de maintenant, nous allons faire une grande utilisation d'indicateurs de santé et de cognition subjectifs. Ils sont calculés sur la base de la première composante principale des items répertoriés dans les tableaux 2 (santé) et 4 (cognition). L'estimation par variables instrumentales fait appel aux items «objectifs» énumérés dans le tableau 3 pour instrumenter l'indicateur de santé subjectif apparaissant dans la dernière colonne du tableau 2. 


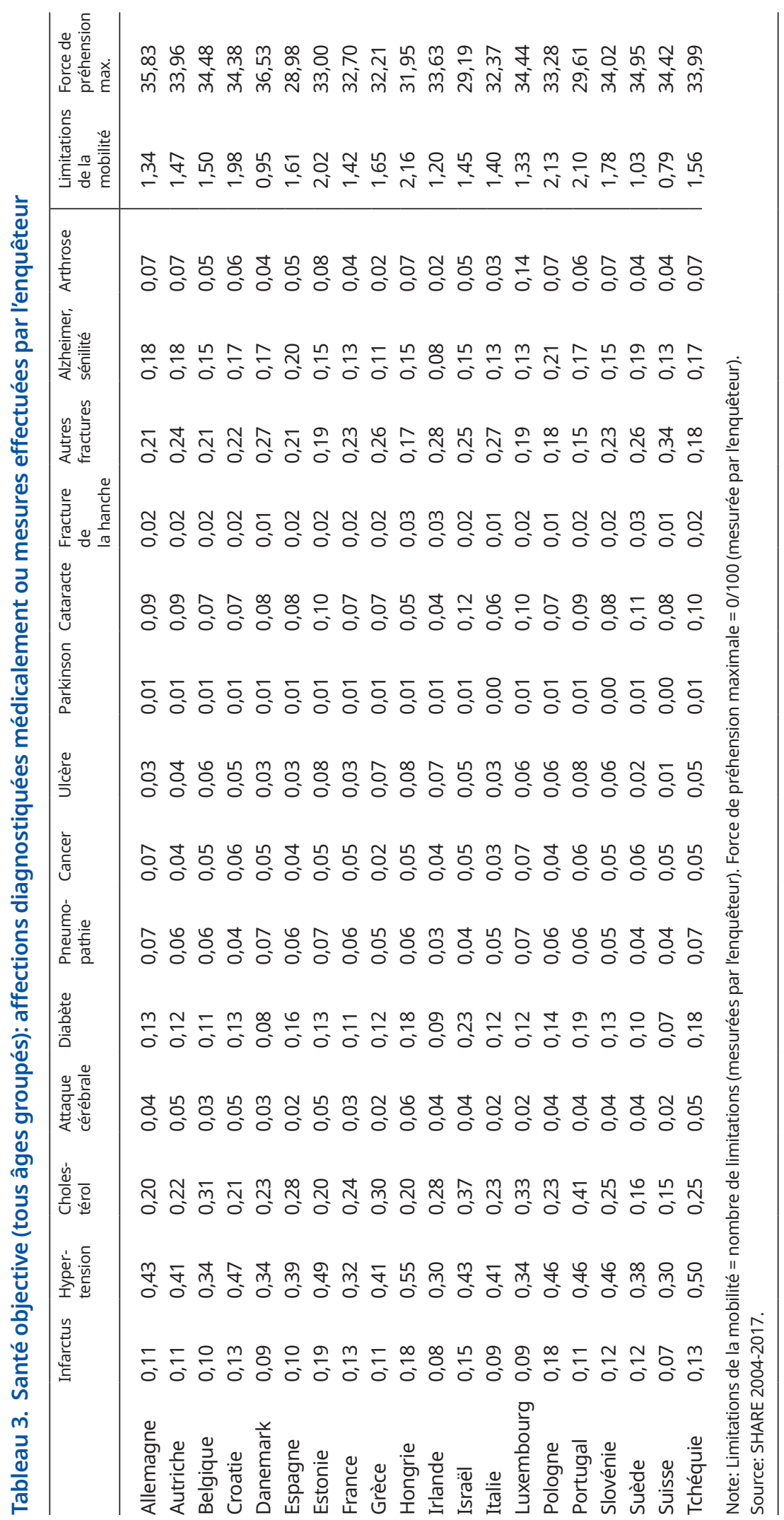



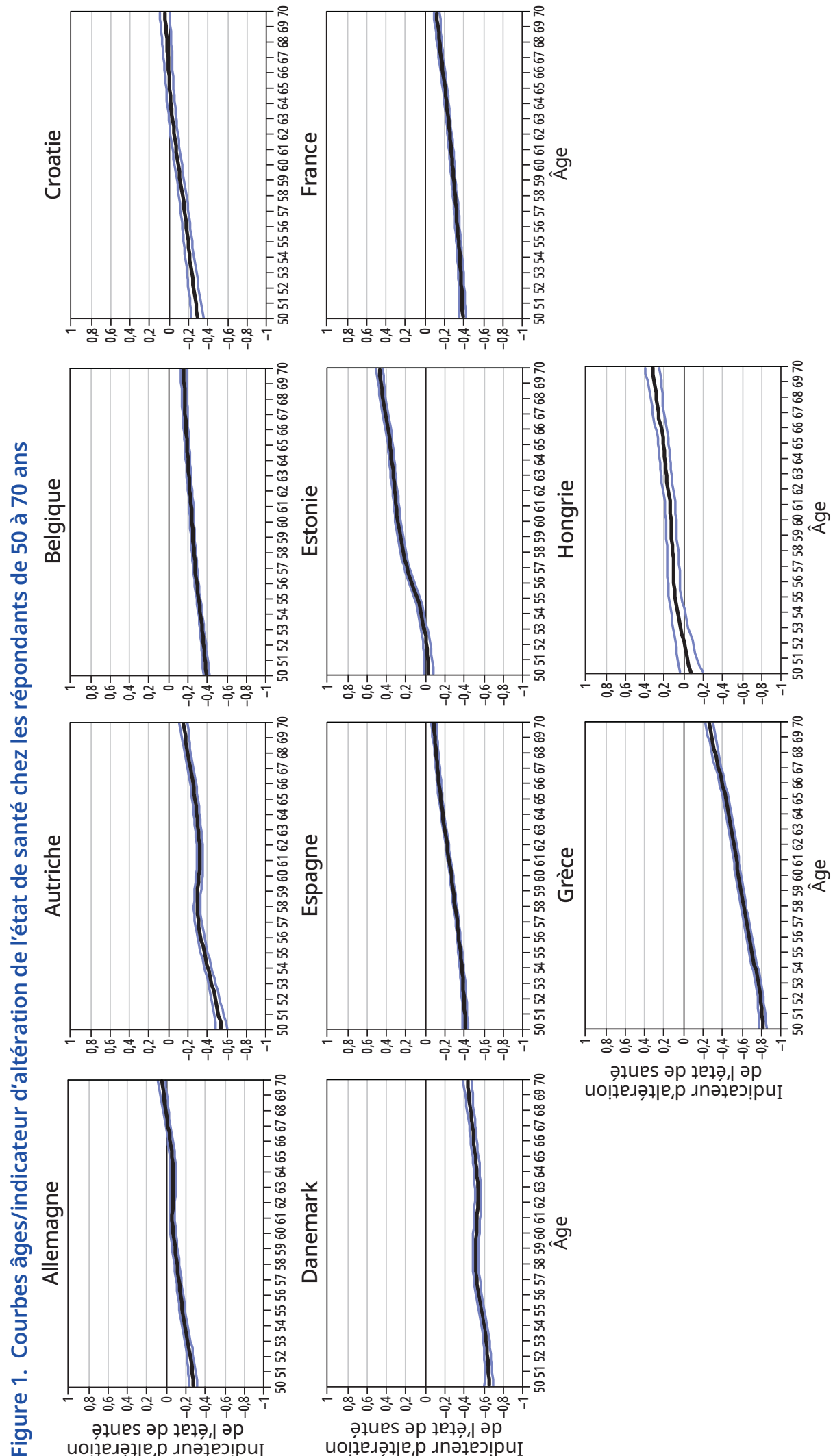

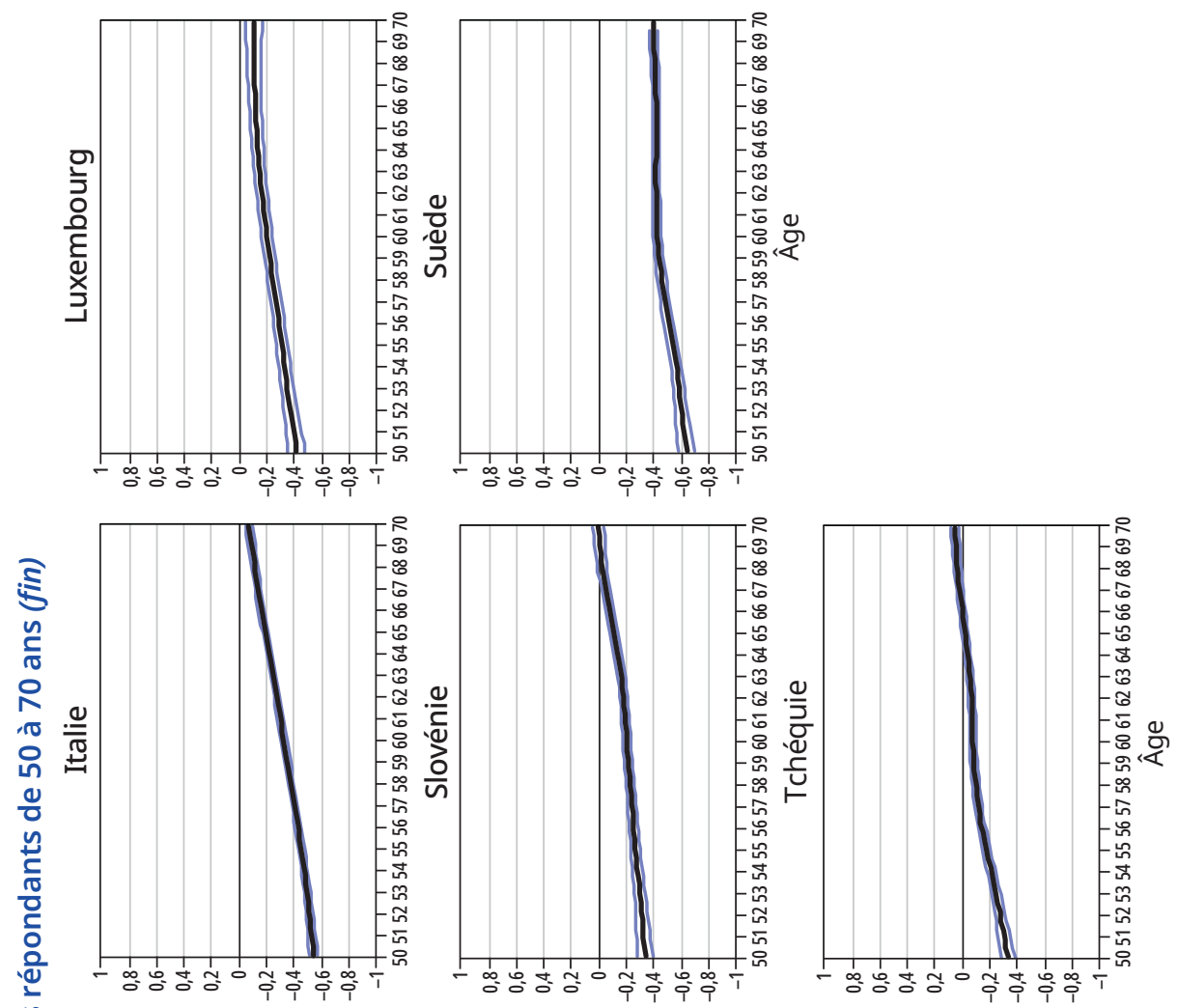

造
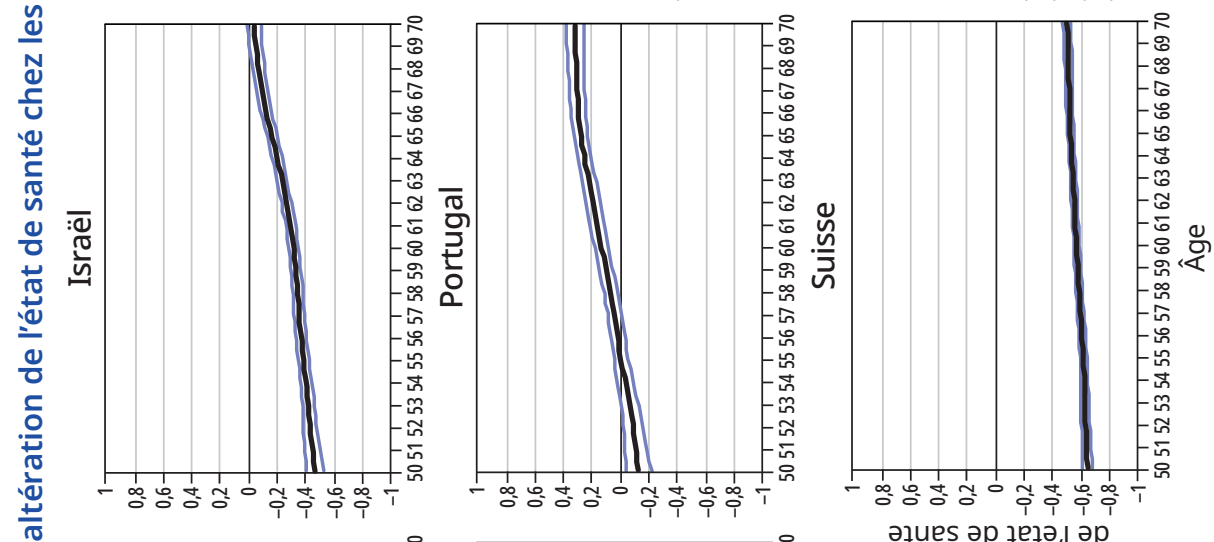

थ

过

$\frac{\Phi}{\sqrt{2}}$

응

要

눈

है 育

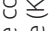

造

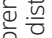

즌

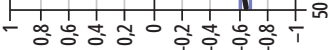

әjues әр رетә әр

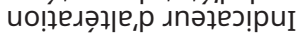

迹

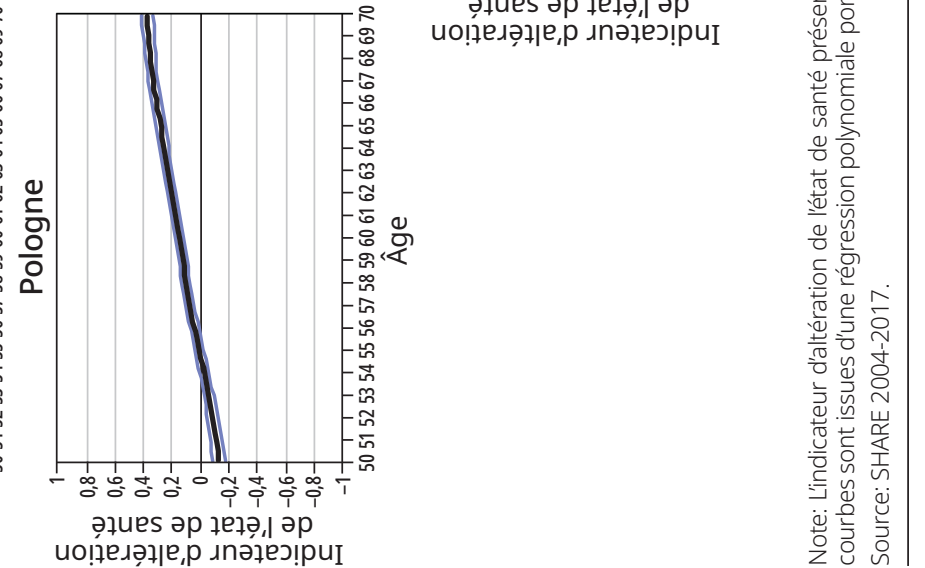



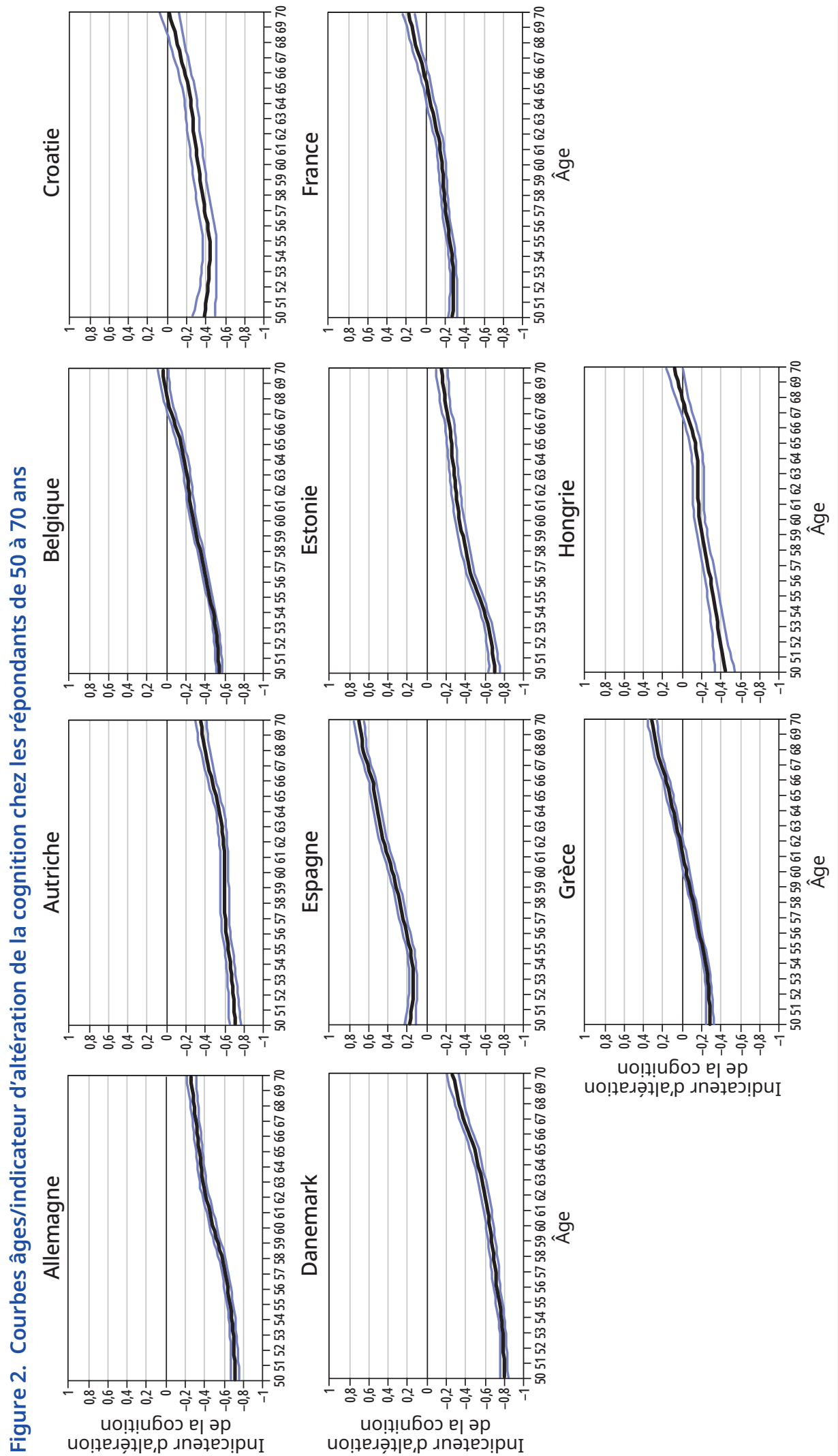

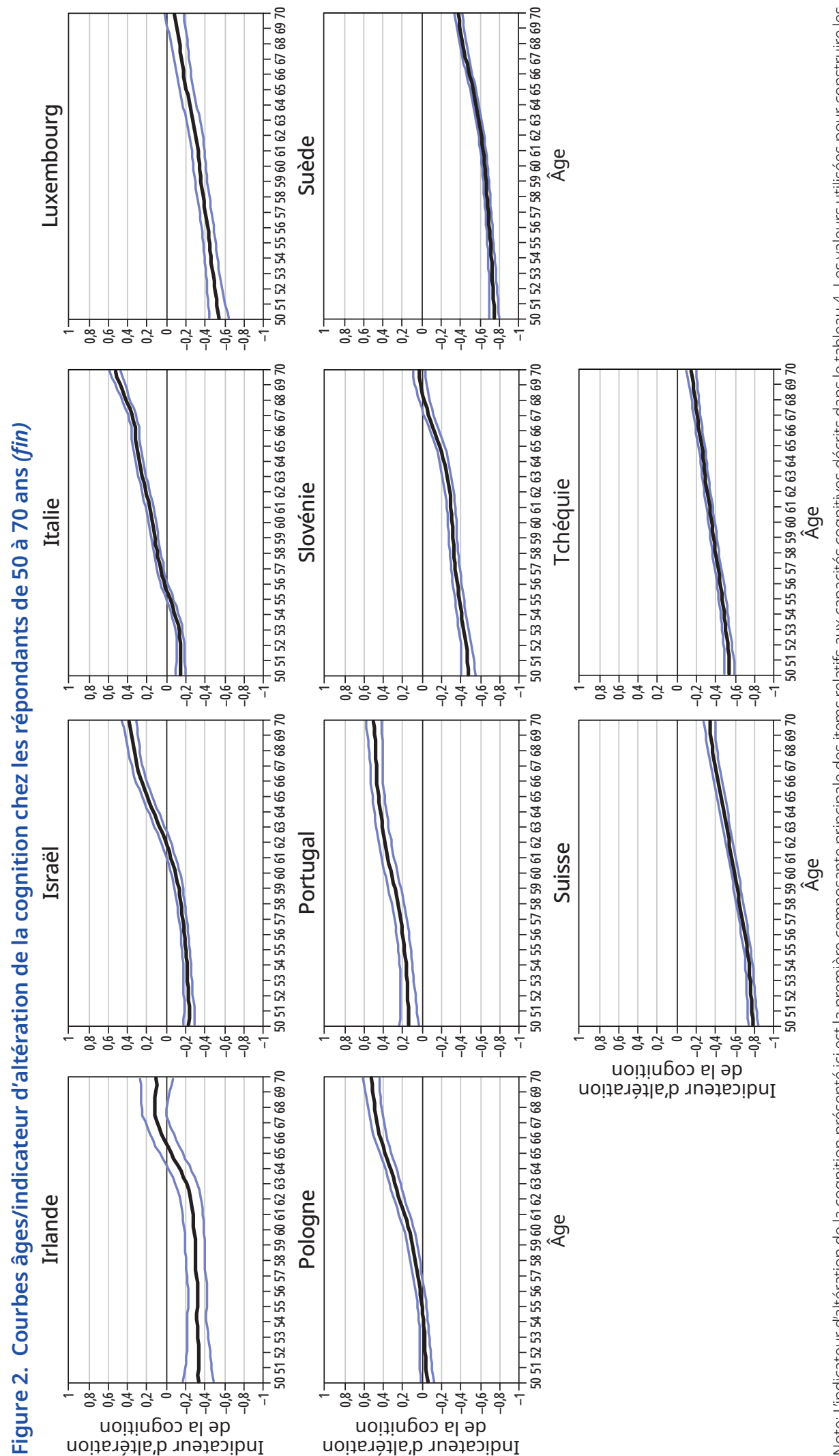

uo!l!uбоว ер әр

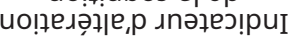

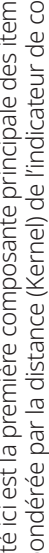

芒 을

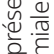

ᄃํำ

흥

ปㄷำ

$\frac{0}{2} \stackrel{\text { Q }}{0}$

ㄷํㅇ ำ

ํㅜㄴ

퐁 궁

च थ

בิ山

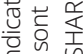

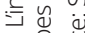

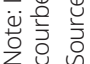


de pair avec une augmentation de 5 pour cent d'un écart type des indicateurs de santé et de cognition. La figure 3 montre qu'une année supplémentaire est systématiquement associée à une dégradation de l'état de santé. Cette dégradation est apparemment plus marquée parmi les personnes de 50 à 54 que parmi celles de 60 à 64 ans. À partir de 70 ans, la dégradation s'accélère cependant clairement. La cognition ne semble pas sensible à l'âge entre 50 et 54 ans, mais elle se dégrade continûment à partir de 55 ans et la corrélation avec l'âge ne fait aucun doute.

Figure 3. Impact marginal du vieillissement sur l'altération de la santé ou de la cognition

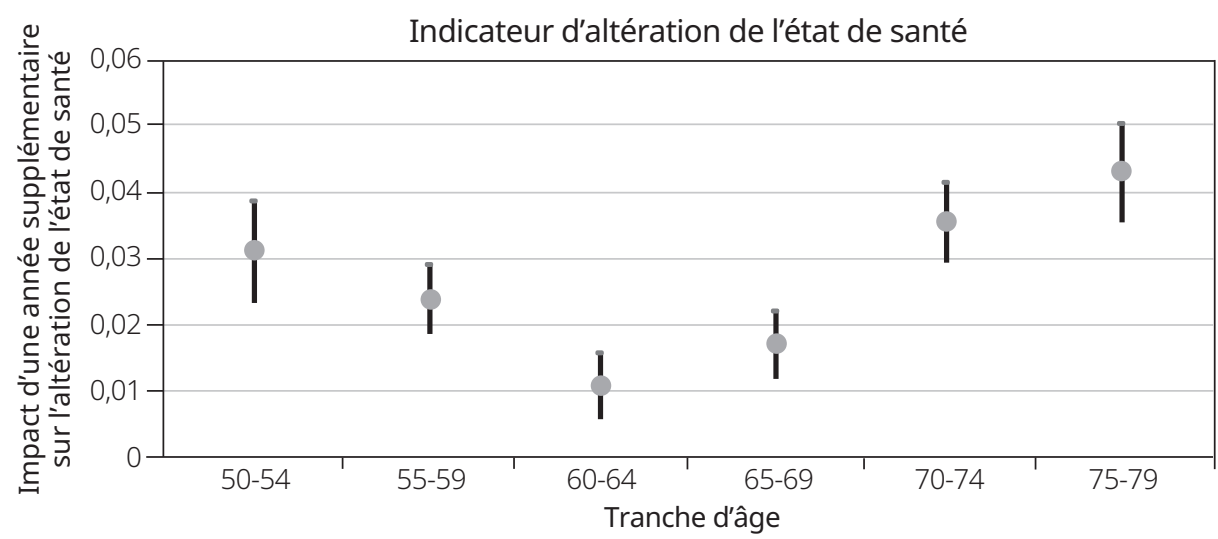

Indicateur d'altération de la cognition

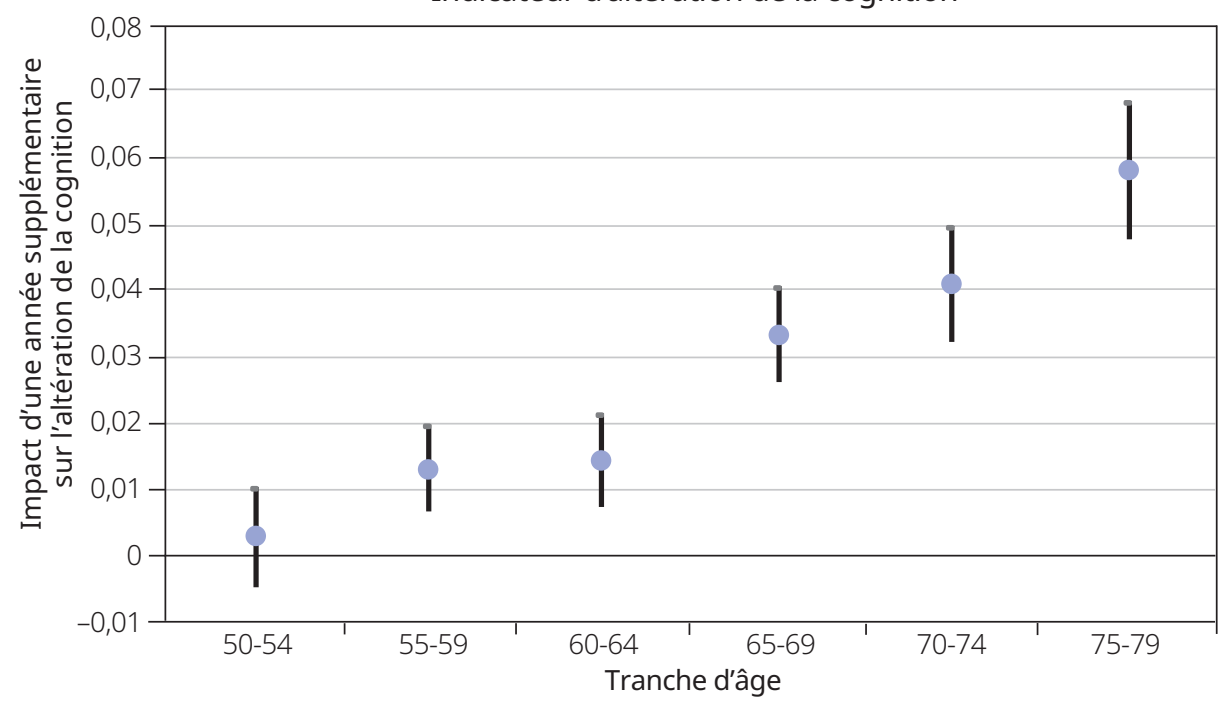

Note: Les points sont des coefficients estimés. Ils rendent compte de l'impact d'une année supplémentaire (au sein de chaque tranche d'âge figurant en abscisse), exprimé en part d'un écart type des indicateurs d'altération de la santé et de la cognition. Les barres verticales représentent les intervalles de confiance à 95 pour cent. Tous les pays sont groupés, mais les équations de régression contiennent l'interaction entre les effets fixes pays, sexe et niveau d'études.

Source: SHARE 2004-2017. 


\section{2. Évaluation de l'impact de la santé ou de la cognition sur le travail au-delà de 54 ans}

\subsubsection{Résultats de la première étape}

Cette partie porte sur la question centrale de l'article, à savoir l'impact potentiel de l'altération de la santé et/ou de la cognition sur la capacité de travail des personnes de 55 à 70 ans.

Le tableau 5 présente les résultats obtenus lors de la première étape lorsque nous utilisons notre modèle préféré - à variables instrumentales -, à savoir que la santé objective est instrumentée par les items «objectifs» répertoriés dans le tableau $3^{10}$. Les résultats des estimations par les MCO sont présentés dans le tableau A1 figurant en annexe. La partie supérieure du tableau 5 (partie A) rend compte de l'impact de l'altération de la santé sur le travail chez les personnes de 50 à 54 ans. La première ligne ( $\beta_{h}^{W O R K}$ ) met en évidence un effet négatif non négligeable de cette altération sur le travail total (WORK), autrement dit sur le nombre total d'heures travaillées au sein de la population âgée de 50 à 54 ans. On observe un coefficient négatif et statistiquement significatif dans tous les pays. Si l'on prend l'exemple de la Suède, le coefficient égal à -8,95 signifie qu'à un écart type supplémentaire dans l'indicateur de santé correspond une diminution de près de neuf heures du nombre moyen d'heures travaillées par la population de 50 à 54 ans. La ligne suivante $\left(\beta_{h}^{E M P L}\right)$ rend compte de l'impact d'un écart type de l'indicateur sur le taux d'emploi (EMPL). Tous les coefficients sont négatifs, statistiquement significatifs et élevés. Si l'on reprend l'exemple de la Suède, une augmentation d'un écart type de l'indicateur de santé va de pair avec une diminution de 18 points de pourcentage du taux d'emploi. La dernière ligne $\left(\beta_{h}^{H O U R S}\right)$ rend compte des résultats obtenus pour la marge intensive. Ces résultats sont tous négatifs, mais la plupart d'entre eux ne sont pas statistiquement significatifs. Cette différence par rapport aux résultats obtenus pour le taux d'emploi EMPL laisse penser que la marge extensive (sortie de l'emploi) est la principale variable d'ajustement utilisée lorsque surviennent des problèmes de santé.

La partie inférieure du tableau 5 (partie B) présente les résultats pour la cognition. Il ne fait aucun doute que la corrélation entre altération de la cognition et travail chez les personnes de 50 à 54 ans est plus faible que la corrélation entre santé et travail, quelle que soit la dimension du travail considérée. Ce résultat est encore plus flagrant sur la figure 4, qui compare les effets sur le travail de l'altération de la santé ( $\beta_{h}{ }^{\text {WORK }}$ ) à ceux de l'altération de la cognition ( $\beta_{c}{ }^{\text {WORK }}$ ). Dans beaucoup de pays, contrairement à l'impact des problèmes de santé, l'impact de l'altération de la cognition entre 50 et 54 ans n'est pas statistiquement significatif, ce qui pourrait indiquer que l’altération de la santé constitue un déterminant beaucoup plus important de l'activité que l'altération de la cognition. Cette conclusion doit toutefois être nuancée parce qu'il est possible que SHARE se prête moins à la mesure de la cognition qu'à celle de la santé, ou du moins à la mesure des composantes de la cognition qui influent sur le travail entre

10 À noter que seule la santé est instrumentée. Nous supposons que l'indicateur de cognition comporte un risque d'endogénéité beaucoup plus faible parce qu'il résulte de scores à des tests administrés par les enquêteurs. 
Tableau 5. Résultats de la première étape (modèle à variables instrumentales): impact de l'altération de l'état de santé et de la cognition sur le travail chez les répondants de 50 à 54 ans

\begin{tabular}{|c|c|c|c|c|c|c|c|c|c|c|}
\hline & Allemagne & Autriche & Belgique & Croatie & Danemark & Espagne & Estonie & France & Grèce & Hongrie \\
\hline & \multicolumn{10}{|c|}{ A. Indicateur d'altération de l'état de santé } \\
\hline$\beta_{h}^{\text {WORK }}$ & $\begin{array}{l}-8,70^{* * * *} \\
(0,000)\end{array}$ & $\begin{array}{l}-10,41^{* * *} \\
(0,000)\end{array}$ & $\begin{array}{l}-7,96^{* * *} \\
(0,000)\end{array}$ & $\begin{array}{l}-8,85^{* * *} \\
(0,000)\end{array}$ & $\begin{array}{l}-9,84^{* * *} \\
(0,000)\end{array}$ & $\begin{array}{l}-8,83^{* * *} \\
(0,000)\end{array}$ & $\begin{array}{l}-9,30 * * * \\
(0,000)\end{array}$ & $\begin{array}{l}-6,27^{* * *} \\
(0,000)\end{array}$ & $\begin{array}{l}-4,24^{* *} \\
(0,006)\end{array}$ & $\begin{array}{l}-11,44^{\text {*** }} \\
(0,000)\end{array}$ \\
\hline$\beta_{h}^{E M P L}$ & $\begin{array}{l}-0,21^{* * *} \\
(0,000)\end{array}$ & $\begin{array}{l}-0,28^{* * *} \\
(0,000)\end{array}$ & $\begin{array}{l}-0,20 * * * \\
(0,000)\end{array}$ & $\begin{array}{l}-0,22^{* * *} \\
(0,000)\end{array}$ & $\begin{array}{l}-0,23^{* * *} \\
(0,000)\end{array}$ & $\begin{array}{l}-0,18^{* * *} \\
(0,000)\end{array}$ & $\begin{array}{l}-0,23^{* * *} \\
(0,000)\end{array}$ & $\begin{array}{l}-0,18^{* * *} \\
(0,000)\end{array}$ & $\begin{array}{l}-0,10 * * * \\
(0,001)\end{array}$ & $\begin{array}{l}-0,30 * * * \\
(0,000)\end{array}$ \\
\hline \multirow[t]{2}{*}{$\beta_{h}^{H O U R S}$} & $\begin{array}{l}-0,58 \\
(0,507)\end{array}$ & $\begin{array}{l}-0,02 \\
(0,995)\end{array}$ & $\begin{array}{l}-1,81^{*} \\
(0,024)\end{array}$ & $\begin{array}{l}-2,42^{* *} \\
(0,003)\end{array}$ & $\begin{array}{l}-2,02 \\
(0,050)\end{array}$ & $\begin{array}{l}-3,24 \\
(0,106)\end{array}$ & $\begin{array}{l}-0,83 \\
(0,285)\end{array}$ & $\begin{array}{l}-0,80 \\
(0,205)\end{array}$ & $\begin{array}{l}-0,84 \\
(0,621)\end{array}$ & $\begin{array}{l}-0,90 \\
(0,286)\end{array}$ \\
\hline & \multicolumn{10}{|c|}{ B. Indicateur d'altération de la cognition } \\
\hline$\beta_{c}^{\text {WORK }}$ & $\begin{array}{l}-0,07 \\
(0,917)\end{array}$ & $\begin{array}{l}-3,34^{*} \\
(0,014)\end{array}$ & $\begin{array}{l}-1,57^{* *} \\
(0,002)\end{array}$ & $\begin{array}{l}1,53 \\
(0,128)\end{array}$ & $\begin{array}{l}-1,00 \\
(0,408)\end{array}$ & $\begin{array}{l}-4,15^{* * *} \\
(0,000)\end{array}$ & $\begin{array}{l}-1,82 \\
(0,125)\end{array}$ & $\begin{array}{l}-3,07^{* * *} \\
(0,000)\end{array}$ & $\begin{array}{l}0,39 \\
(0,722)\end{array}$ & $\begin{array}{l}-1,15 \\
(0,600)\end{array}$ \\
\hline$\beta_{c}^{E M P L}$ & $\begin{array}{l}-0,04^{* * *} \\
(0,000)\end{array}$ & $\begin{array}{l}-0,05 \\
(0,174)\end{array}$ & $\begin{array}{l}-0,06^{* * * *} \\
(0,000)\end{array}$ & $\begin{array}{l}0,06 * \\
(0,014)\end{array}$ & $\begin{array}{l}-0,05 \\
(0,053)\end{array}$ & $\begin{array}{l}-0,10^{* * *} \\
(0,000)\end{array}$ & $\begin{array}{l}-0,03 \\
(0,223)\end{array}$ & $\begin{array}{l}-0,04^{* *} \\
(0,002)\end{array}$ & $\begin{array}{l}-0,01 \\
(0,811)\end{array}$ & $\begin{array}{l}-0,05^{* * *} \\
(0,000)\end{array}$ \\
\hline$\beta_{c}^{\text {HOURS }}$ & $\begin{array}{l}1,25^{*} \\
(0,037)\end{array}$ & $\begin{array}{l}-2,54^{* *} \\
(0,008)\end{array}$ & $\begin{array}{l}0,40 \\
(0,271)\end{array}$ & $\begin{array}{l}-0,25 \\
(0,776)\end{array}$ & $\begin{array}{l}-0,70 \\
(0,209)\end{array}$ & $\begin{array}{l}-0,84^{*} \\
(0,039)\end{array}$ & $\begin{array}{l}-0,04 \\
(0,909)\end{array}$ & $\begin{array}{l}-2,15^{*} \\
(0,015)\end{array}$ & $\begin{array}{l}0,65 \\
(0,631)\end{array}$ & $\begin{array}{l}1,65 \\
(0,334)\end{array}$ \\
\hline \multirow[t]{2}{*}{$\mathrm{N}$} & 20680 & 18373 & 27750 & 4822 & 17206 & 24474 & 22369 & 22644 & 13710 & 4523 \\
\hline & Irlande & Israël & Italie & Luxembourg & Pologne & Portugal & Slovénie & Suède & Suisse & Tchéquie \\
\hline & \multicolumn{10}{|c|}{ A. Indicateur d'altération de l'état de santé } \\
\hline$\beta_{h}^{\text {WORK }}$ & $\begin{array}{l}-6,76^{* *} \\
(0,003)\end{array}$ & $\begin{array}{l}-9,00 * * * \\
(0,000)\end{array}$ & $\begin{array}{l}-5,47^{* * * *} \\
(0,000)\end{array}$ & $\begin{array}{l}-6,26 \\
(0,065)\end{array}$ & $\begin{array}{l}-11,72^{* * * *} \\
(0,000)\end{array}$ & $\begin{array}{l}-6,23^{* * *} \\
(0,000)\end{array}$ & $\begin{array}{l}-8,95^{* * *} \\
(0,000)\end{array}$ & $\begin{array}{l}-8,95^{* * *} \\
(0,000)\end{array}$ & $\begin{array}{l}-4,46 * * * \\
(0,000)\end{array}$ & $\begin{array}{l}-12,22^{\text {*** }} \\
(0,000)\end{array}$ \\
\hline$\beta_{h}^{E M P L}$ & $\begin{array}{l}-0,19 * \\
(0,028)\end{array}$ & $\begin{array}{l}-0,23^{* * * *} \\
(0,000)\end{array}$ & $\begin{array}{l}-0,12^{* * *} \\
(0,000)\end{array}$ & $\begin{array}{l}-0,12 \\
(0,190)\end{array}$ & $\begin{array}{l}-0,24^{* * *} \\
(0,000)\end{array}$ & $\begin{array}{l}-0,23^{* * *} \\
(0,000)\end{array}$ & $\begin{array}{l}-0,17^{* * *} \\
(0,000)\end{array}$ & $\begin{array}{l}-0,18^{* * *} \\
(0,000)\end{array}$ & $\begin{array}{l}-0,14^{* *} \\
(0,002)\end{array}$ & $\begin{array}{l}-0,29 * * * \\
(0,000)\end{array}$ \\
\hline \multirow[t]{2}{*}{$\beta_{h}^{\text {HOURS }}$} & $\begin{array}{l}-7,55 \\
(0,093)\end{array}$ & $\begin{array}{l}0,71 \\
(0,581)\end{array}$ & $\begin{array}{l}-2,91^{* * * *} \\
(0,000)\end{array}$ & $\begin{array}{l}-2,22 \\
(0,435)\end{array}$ & $\begin{array}{l}0,23 \\
(0,934)\end{array}$ & $\begin{array}{l}-3,23 \\
(0,585)\end{array}$ & $\begin{array}{l}-3,53^{* *} \\
(0,009)\end{array}$ & $\begin{array}{l}-2,15^{*} \\
(0,012)\end{array}$ & $\begin{array}{l}1,20 \\
(0,425)\end{array}$ & $\begin{array}{l}-1,02 \\
(0,680)\end{array}$ \\
\hline & \multicolumn{10}{|c|}{ B. Indicateur d'altération de la cognition } \\
\hline$\beta_{c}^{\text {WORK }}$ & $\begin{array}{l}-2,57 \\
(0,398)\end{array}$ & $\begin{array}{l}-1,26 \\
(0,556)\end{array}$ & $\begin{array}{l}-3,99 * * * \\
(0,000)\end{array}$ & $\begin{array}{l}0,65 \\
(0,630)\end{array}$ & $\begin{array}{l}-0,75 \\
(0,478)\end{array}$ & $\begin{array}{l}0,24 \\
(0,904)\end{array}$ & $\begin{array}{l}-1,56 \\
(0,239)\end{array}$ & $\begin{array}{l}0,50 \\
(0,105)\end{array}$ & $\begin{array}{l}-2,10 * * * \\
(0,001)\end{array}$ & $\begin{array}{l}0,03 \\
(0,984)\end{array}$ \\
\hline$\beta_{c}^{E M P L}$ & $\begin{array}{l}0,08 \\
(0,175)\end{array}$ & $\begin{array}{l}-0,03 \\
(0,455)\end{array}$ & $\begin{array}{l}-0,09 * * * \\
(0,000)\end{array}$ & $\begin{array}{l}0,01 \\
(0,799)\end{array}$ & $\begin{array}{l}-0,04 \\
(0,103)\end{array}$ & $\begin{array}{l}0,02 \\
(0,810)\end{array}$ & $\begin{array}{l}-0,03 \\
(0,150)\end{array}$ & $\begin{array}{l}0,02 \\
(0,242)\end{array}$ & $\begin{array}{l}-0,03 \\
(0,161)\end{array}$ & $\begin{array}{l}-0,00 \\
(0,873)\end{array}$ \\
\hline$\beta_{c}^{\text {HOURS }}$ & $\begin{array}{l}-6,87^{* *} \\
(0,001)\end{array}$ & $\begin{array}{l}-1,59 \\
(0,491)\end{array}$ & $\begin{array}{l}-2,37^{* *} \\
(0,006)\end{array}$ & $\begin{array}{l}0,85 \\
(0,582)\end{array}$ & $\begin{array}{l}0,65 \\
(0,397)\end{array}$ & $\begin{array}{l}2,03 \\
(0,611)\end{array}$ & $\begin{array}{l}-0,23 \\
(0,822)\end{array}$ & $\begin{array}{l}0,04 \\
(0,950)\end{array}$ & $\begin{array}{l}-0,33 \\
(0,653)\end{array}$ & $\begin{array}{l}-0,19 \\
(0,850)\end{array}$ \\
\hline $\mathrm{N}$ & 1003 & 10880 & 23137 & 4372 & 10265 & 4010 & 13478 & 18772 & 13966 & 22209 \\
\hline
\end{tabular}

Note: Les symboles *,** et *** correspondent respectivement aux seuils de significativité statistique de 10 pour cent, 5 pour cent et 1 pour cent. Estimations ponctuelles $\beta_{h}^{Z}$ et $\beta_{c}^{Z}$ de l'effet d'une augmentation d'un écart type de l'indicateur sur le travail, l'emploi et le nombre d'heures travaillées. Les valeurs $p$ sont indiquées entre parenthèses. Les erreurs type sous-jacentes ont été estimées au moyen de la méthode bootstrap (100 itérations). L'indicateur de santé subjectif/physique a été instrumenté par les variables objectives figurant dans le tableau 3. Le travail total (WORK) résulte de la combinaison du nombre d'heures travaillées (HOURS) et de l'emploi (EMPL).

Source: Calculs réalisés par l'auteur sur la base des données issues de SHARE (2004-2017).

50 et 54 ans. On pourrait avancer que les items figurant dans le tableau 4 correspondent à des problèmes qui n’apparaissent qu’à un âge relativement avancé. Cependant, la figure 3 montre que les items de l'enquête qui se rapportent à la cognition rendent compte d'évolutions des capacités cognitives qui se produisent beaucoup plus tôt. Dès la tranche d'âge 55-59 ans, l'impact 
Figure 4. Comparaison entre l'impact de l'altération de l'état de santé $\left(\beta_{h}^{\text {WORK }}\right)$ et l'impact de l'altération de la cognition $\left(\beta_{c}^{\text {WORK }}\right)$ sur le travail total chez les répondants âgés de 50 à 54 ans

Coefficient d'altération de l'état de santé

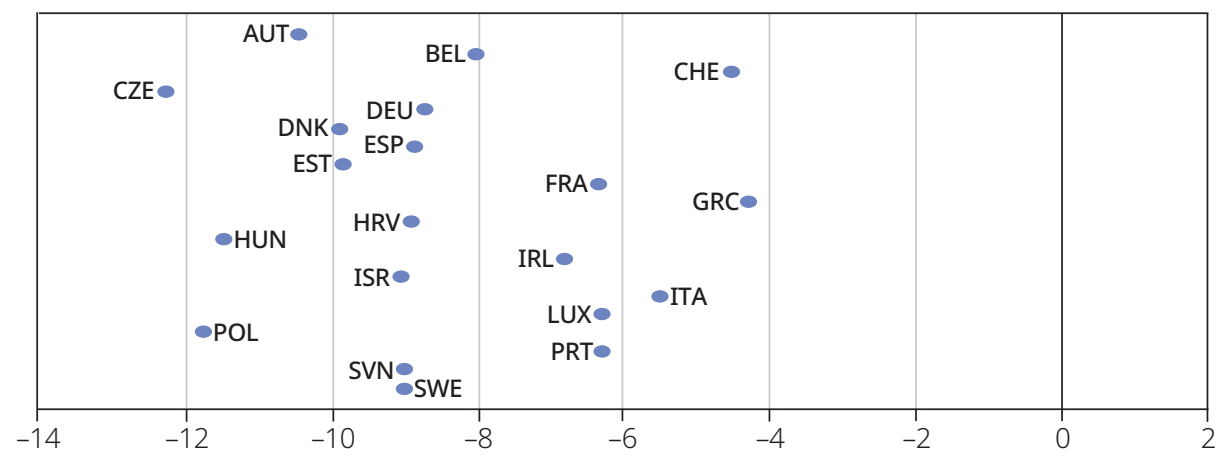

Coefficient d'altération de la cognition

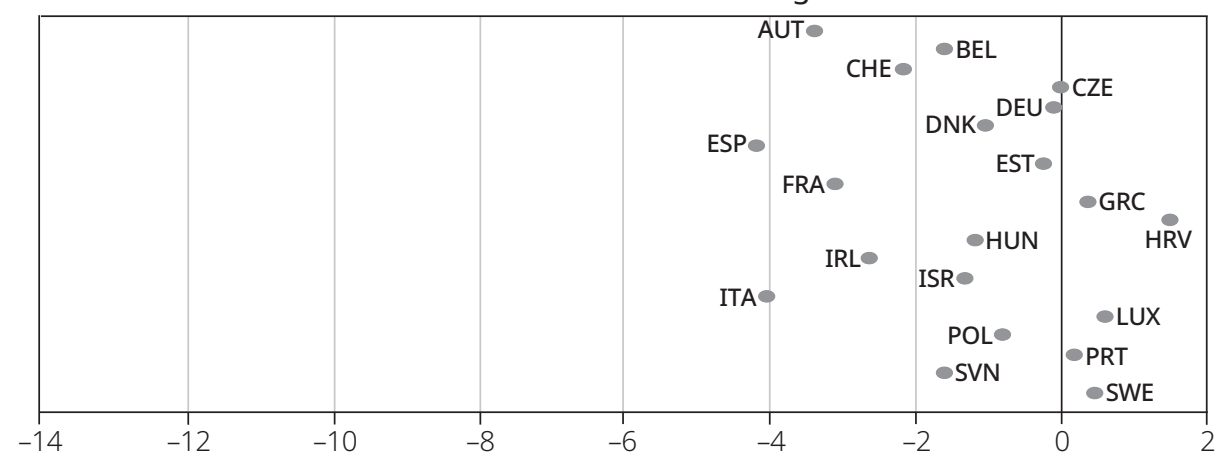

Note: Les pays sont désignés par leur code ISO 3166-1 alpha-3.

Source: Calculs réalisés par l'auteur sur la base des données issues de SHARE (2004-2017).

marginal du vieillissement sur l'indicateur de la cognition est positif et statistiquement significatif.

Avant de passer aux résultats de la deuxième étape, soulignons que la correction de l'endogénéité dans la première étape semble jouer un rôle important. Une comparaison des estimations obtenues dans le modèle à variables instrumentales avec celles obtenues par les MCO en ce qui concerne l'altération de la santé (figure 5) montre que l'estimation par les MCO conduit à sous-estimer l'impact négatif des problèmes de santé sur le travail au sein de la population de 50 à 54 ans. Dans le cas de la Suède, la diminution du travail total (WORK) s'établit à 8,95 heures quand on utilise le modèle à variables instrumentales et à 6,78 heures seulement d'après les estimations par les MCO. La différence constatée entre les deux méthodes d'estimation est similaire dans les autres pays, ce qui semble indiquer qu'il n'existe pas de biais de justification fort. Ces résultats laissent penser qu'il existe des erreurs de mesure inhérentes à l'absence d'indicateur adapté du «stock» de santé global et qu'il s'ensuit un biais d'atténuation. Le tableau A2 en annexe présente les résultats détaillés d'une comparaison entre la baisse prédite du travail total (WORK) obtenue avec le modèle à variables 
Figure 5. Impact de l'altération de la santé sur le travail total ( $\left.\beta^{\text {WORK }}\right)$ chez les répondants âgés de 50 à 54 ans - comparaison entre les résultats issus du modèle à variables instrumentales et les estimations par les MCO

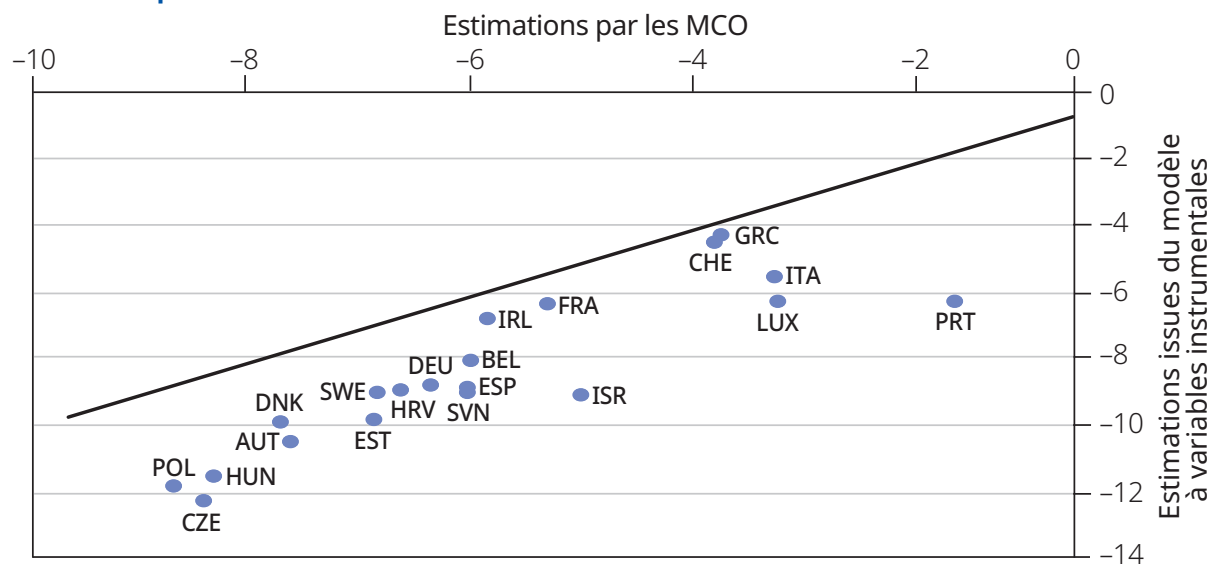

Source: Calculs réalisés par l'auteur sur la base des données issues de SHARE (2004-2017).

instrumentales et cette même baisse d'après les estimations par les MCO. Le tableau A3 présente les résultats des tests de validité des instruments et des tests de sous-identification. Pour tous les pays, nous rejetons les corrélations nulles ou non significatives statistiquement entre l'indicateur de santé subjectif et les indicateurs objectifs. Cet exercice montre que les indicateurs «objectifs» sont des prédicteurs forts de l'indicateur subjectif. Enfin, le tableau A4 en annexe présente les résultats de la comparaison entre deux spécifications du modèle à variables instrumentales, l'une n'incluant que l'altération de la santé et l'autre l'altération de la santé et de la cognition. Cette comparaison ne démontre pas la supériorité de la spécification plus complète incluant la cognition. Dans ce modèle, les coefficients obtenus pour l'indicateur de santé sont à peine différents de ceux résultant du modèle plus simple.

\subsubsection{Résultats de la deuxième étape}

Le tableau 6 présente les résultats de la deuxième étape, obtenus à partir des coefficients calculés lors de la première étape avec le modèle à variables instrumentales (tableau 5). Ils consistent essentiellement en prédictions de ce que serait la capacité de travail des personnes de 70 ans si la santé et la cognition étaient les seuls déterminants du travail. Ils sont exprimés sous la forme d'un écart par rapport à la capacité de travail des individus de 50 ans. La partie $\mathrm{A}$ du tableau montre que, dans le cas de la Suède, le taux d'emploi diminuerait de 4,08 points tandis que le travail total diminuerait de 2,193 heures. La partie B présente les mêmes calculs, mais en termes relatifs. La partie $C$, qui est peut-être plus intéressante, contient les estimations de $\delta^{Z, 50-70}$ (équations (3) à (5) présentées dans la partie 3), autrement dit de la part de la diminution du travail observée entre 50 et 70 ans qui est imputable à la baisse de la capacité de travail due à la santé ou à la cognition. Dans le cas de la Suède, cette baisse n'explique 
Tableau 6. Résultats de la deuxième étape: diminution du travail parmi les personnes de 70 ans (par rapport aux personnes de 50 ans) expliquée par l'altération de l'état de santé et/ou de la cognition

\begin{tabular}{|c|c|c|c|c|c|c|c|c|c|c|}
\hline & Allemagne & Autriche & Belgique & Croatie & Danemark & Espagne & Estonie & France & Grèce & Hongrie \\
\hline & \multicolumn{10}{|c|}{$\begin{array}{l}\text { A. Niveau de diminution prédite par l'altération de l'état de santé et/ou de la cognition } \\
(0=\text { niveau chez les personnes de } 50 \text { à } 54 \text { ans) }\end{array}$} \\
\hline WORK & $\begin{array}{l}-2,660 * * * \\
(0,000)\end{array}$ & $\begin{array}{l}-3,068^{* * *} \\
(0,000)\end{array}$ & $\begin{array}{l}-4,224^{* * *} \\
(0,000)\end{array}$ & $\begin{array}{l}-3,875^{* *} \\
(0,003)\end{array}$ & $\begin{array}{l}-3,887^{* * *} \\
(0,000)\end{array}$ & $\begin{array}{l}-5,347^{* * *} \\
(0,000)\end{array}$ & $\begin{array}{l}-6,201 * * * \\
(0,000)\end{array}$ & $\begin{array}{l}-4,112 * * * \\
(0,000)\end{array}$ & $\begin{array}{l}-0,460 \\
(0,660)\end{array}$ & $\begin{array}{l}-6,566 * * * \\
(0,000)\end{array}$ \\
\hline$E M P L$ & $\begin{array}{l}-0,107^{* * *} \\
(0,000)\end{array}$ & $\begin{array}{l}-0,0778^{* * *} \\
(0,000)\end{array}$ & $\begin{array}{l}-0,122^{* * *} \\
(0,000)\end{array}$ & $\begin{array}{l}-0,0865^{* *} \\
(0,004)\end{array}$ & $\begin{array}{l}-0,101^{* * *} \\
(0,000)\end{array}$ & $\begin{array}{l}-0,131^{* * * *} \\
(0,000)\end{array}$ & $\begin{array}{l}-0,140 * * * \\
(0,000)\end{array}$ & $\begin{array}{l}-0,0980^{* * * *} \\
(0,000)\end{array}$ & $\begin{array}{l}-0,0288 \\
(0,196)\end{array}$ & $\begin{array}{l}-0,176^{* * * *} \\
(0,000)\end{array}$ \\
\hline \multirow[t]{2}{*}{ HOURS } & $\begin{array}{l}1,471^{* *} \\
(0,001)\end{array}$ & $\begin{array}{l}-0,372 \\
(0,487)\end{array}$ & $\begin{array}{l}-0,128 \\
(0,746)\end{array}$ & $\begin{array}{l}-0,457 \\
(0,402)\end{array}$ & $\begin{array}{l}-0,0539 \\
(0,901)\end{array}$ & $\begin{array}{l}-1,701^{*} \\
(0,023)\end{array}$ & $\begin{array}{l}-0,855 \\
(0,080)\end{array}$ & $\begin{array}{l}-1,297^{* * *} \\
(0,001)\end{array}$ & $\begin{array}{l}0,296 \\
(0,778)\end{array}$ & $\begin{array}{l}0,561 \\
(0,641)\end{array}$ \\
\hline & \multicolumn{10}{|c|}{$\begin{array}{l}\text { B. Pourcentage de diminution prédite par l'altération de l'état de santé et/ou de la cognition } \\
(1=\text { niveau chez les personnes de } 50 \text { à } 54 \text { ans) }\end{array}$} \\
\hline WORK & $\begin{array}{l}-0,0951^{* * *} \\
(0,000)\end{array}$ & $\begin{array}{l}-0,111^{* * *} \\
(0,000)\end{array}$ & $\begin{array}{l}-0,151^{* * * *} \\
(0,000)\end{array}$ & $\begin{array}{l}-0,160 * * \\
(0,002)\end{array}$ & $\begin{array}{l}-0,120 * * * \\
(0,000)\end{array}$ & $\begin{array}{l}-0,213^{* * *} \\
(0,000)\end{array}$ & $\begin{array}{l}-0,195 * * * \\
(0,000)\end{array}$ & $\begin{array}{l}-0,136 * * * \\
(0,000)\end{array}$ & $\begin{array}{l}-0,0201 \\
(0,659)\end{array}$ & $\begin{array}{l}-0,246^{* * *} \\
(0,000)\end{array}$ \\
\hline EMPL & $-0,141^{* * * *}$ & $-0,109 * * *$ & $-0,162^{* * *}$ & $-0,152^{* *}$ & $-0,119^{* * * *}$ & $-0,199 * * *$ & $-0,178^{* * *}$ & $-0,121 * * *$ & $-0,0492$ & $-0,269^{* * *}$ \\
\hline \multirow[t]{2}{*}{ HOURS } & $\begin{array}{l}0,0531^{* * *} \\
(0,000)\end{array}$ & $\begin{array}{l}-0,00195 \\
(0,890)\end{array}$ & $\begin{array}{l}0,0126 \\
(0,338)\end{array}$ & $\begin{array}{l}-0,00897 \\
(0,557)\end{array}$ & $\begin{array}{l}-0,00119 \\
(0,930)\end{array}$ & $\begin{array}{l}-0,0171 \\
(0,399)\end{array}$ & $\begin{array}{l}-0,0214 \\
(0,113)\end{array}$ & $\begin{array}{l}-0,0164 \\
(0,130)\end{array}$ & $\begin{array}{l}0,0306 \\
(0,291)\end{array}$ & $\begin{array}{l}0,0326 \\
(0,380)\end{array}$ \\
\hline & \multicolumn{10}{|c|}{$\begin{array}{l}\text { C. Part de la diminution du travail observée imputable à l'altération de l'état de santé et/ou de la cognition } \\
(1=100 \%), \delta z, 50-70\end{array}$} \\
\hline WORK & $\begin{array}{l}0,102^{* * *} \\
(0,000)\end{array}$ & $\begin{array}{l}0,117^{* * *} \\
(0,000)\end{array}$ & $\begin{array}{l}0,174 * * * \\
(0,000)\end{array}$ & $\begin{array}{l}0,255^{* *} \\
(0,002)\end{array}$ & $\begin{array}{l}0,140 * * * \\
(0,000)\end{array}$ & $\begin{array}{l}0,229 * * * \\
(0,000)\end{array}$ & $\begin{array}{l}0,256 * * * \\
(0,000)\end{array}$ & $\begin{array}{l}0,149 * * * \\
(0,000)\end{array}$ & $\begin{array}{l}0,0229 \\
(0,661)\end{array}$ & $\begin{array}{l}0,272 * * * \\
(0,000)\end{array}$ \\
\hline EMPL & $\begin{array}{l}0,142^{* * *} \\
(0,000)\end{array}$ & $\begin{array}{l}0,109 * * * \\
(0,000)\end{array}$ & $\begin{array}{l}0,165^{* * *} \\
(0,000)\end{array}$ & $\begin{array}{l}0,150 * * \\
(0,004)\end{array}$ & $\begin{array}{l}0,125^{* * *} \\
(0,000)\end{array}$ & $\begin{array}{l}0,203^{* * *} \\
(0,000)\end{array}$ & $\begin{array}{l}0,207^{* * *} \\
(0,000)\end{array}$ & $\begin{array}{l}0,124^{* * *} \\
(0,000)\end{array}$ & $\begin{array}{l}0,0499 \\
(0,193)\end{array}$ & $\begin{array}{l}0,269 * * * \\
(0,000)\end{array}$ \\
\hline HOURS & $\begin{array}{l}-0,225 \\
(0,950)\end{array}$ & $\begin{array}{l}0,0245 \\
(0,551)\end{array}$ & $\begin{array}{l}0,0150 \\
(0,947)\end{array}$ & $\begin{array}{l}-0,0377 \\
(0,615)\end{array}$ & $\begin{array}{l}0,00547 \\
(0,914)\end{array}$ & $\begin{array}{l}0,206 \\
(0,926)\end{array}$ & $\begin{array}{l}0,101 \\
(0,097)\end{array}$ & $\begin{array}{l}-2,949 \\
(0,880)\end{array}$ & $\begin{array}{l}0,0999 \\
(0,933)\end{array}$ & $\begin{array}{l}-0,108 \\
(0,863)\end{array}$ \\
\hline $\mathrm{N}$ & 20680 & 18373 & 2775 & 4822 & 17206 & 24474 & 22369 & 22644 & 13710 & 4523 \\
\hline & Irlande & Israël & Italie & Luxembourg & Pologne & Portugal & Slovénie & Suède & Suisse & Tchéquie \\
\hline
\end{tabular}

A. Niveau de diminution prédite par l'altération de l'état de santé et/ou de la cognition ( 0 = niveau chez les personnes de 50 à 54 ans)

$\begin{array}{lllllllllll}\text { WORK } & -5,307 & -5,735^{* * *} & -4,192^{* * *} & 0,510 & -8,107^{* * *} & 0,942 & -5,368^{* * *} & -2,193^{* * *} & -0,623 & -6,785^{* * *} \\ & (0,092) & (0,000) & (0,000) & (0,735) & (0,000) & (0,455) & (0,000) & (0,000) & (0,468) & (0,000) \\ \text { EMPL } & -0,0812 & -0,174^{* * *} & -0,112^{* * *} & -0,0233 & -0,240^{* * *} & -0,0965^{*} & -0,120^{* * *} & -0,0408^{* * *} & -0,0605^{* * *} & -0,155^{* * *} \\ & (0,112) & (0,000) & (0,000) & (0,403) & (0,000) & (0,011) & (0,000) & (0,001) & (0,000) & (0,000) \\ \text { HOURS } & -4,743 & 0,0482 & -0,913 & 1,266 & 2,664^{*} & 4,054^{*} & -1,234 & -0,488 & 1,680^{*} & -1,185^{*} \\ & (0,124) & (0,976) & (0,123) & (0,206) & (0,014) & (0,017) & (0,052) & (0,345) & (0,022) & (0,032)\end{array}$

B. Pourcentage de diminution prédite par l'altération de l'état de santé et/ou de la cognition ( 1 = niveau chez les personnes de 50 à 54 ans)

\begin{tabular}{lllllllllll} 
WORK & $-0,186$ & $-0,234^{* * *}$ & $-0,164^{* * *}$ & 0,0179 & $-0,311^{* * *}$ & 0,0893 & $-0,181^{* * *}$ & $-0,0611^{* * *}$ & $-0,0210$ & $-0,204^{* * *}$ \\
& $(0,074)$ & $(0,000)$ & $(0,000)$ & $(0,737)$ & $(0,000)$ & $(0,457)$ & $(0,000)$ & $(0,000)$ & $(0,467)$ & $(0,000)$ \\
EMPL & $-0,115$ & $-0,264^{* * *}$ & $-0,159^{* * *}$ & $-0,0303$ & $-0,383^{* * *}$ & $-0,153^{* *}$ & $-0,161^{* * *}$ & $-0,0458^{* * *}$ & $-0,0722^{* * *}$ & $-0,192^{* * *}$ \\
& $(0,106)$ & $(0,000)$ & $(0,000)$ & $(0,400)$ & $(0,000)$ & $(0,009)$ & $(0,000)$ & $(0,001)$ & $(0,000)$ & $(0,000)$ \\
\multirow{2}{*}{ HOURS } & $-0,0805$ & 0,0413 & $-0,00603$ & 0,0497 & $0,117^{* *}$ & $0,287^{*}$ & $-0,0248$ & $-0,0160$ & $0,0553^{*}$ & $-0,0152$ \\
& $(0,286)$ & $(0,384)$ & $(0,703)$ & $(0,130)$ & $(0,002)$ & $(0,023)$ & $(0,119)$ & $(0,260)$ & $(0,013)$ & $(0,302)$
\end{tabular}

C. Part de la diminution du travail observée imputable à l'altération de l'état de santé et/ou de la cognition $(1=100 \%), \delta z, 50-70$

$\begin{array}{lllllllllll}\text { WORK } & 0,200 & 0,263^{* * *} & 0,193^{* * *} & -0,0205 & 0,638^{* * *} & -0,0791 & 0,205^{* * *} & 0,0661^{* * *} & 0,0238 & 0,223^{* * *} \\ & (0,071) & (0,000) & (0,000) & (0,738) & (0,000) & (0,458) & (0,000) & (0,000) & (0,468) & (0,000) \\ \text { EMPL } & 0,124 & 0,313^{* * *} & 0,167^{* * *} & 0,0306 & 0,355^{* * *} & 0,157^{* *} & 0,163^{* * *} & 0,0476^{* * *} & 0,0781^{* * *} & 0,197^{* * *} \\ & (0,107) & (0,000) & (0,000) & (0,402) & (0,000) & (0,009) & (0,000) & (0,001) & (0,000) & (0,000) \\ \text { HOURS } & 1,167 & -0,00232 & 0,175 & -0,135 & -0,145^{*} & 1,275 & 0,0599 & 0,0248 & -0,128^{*} & 0,129 \\ & (0,733) & (0,975) & (0,938) & (0,830) & (0,015) & (0,870) & (0,338) & (0,370) & (0,036) & (0,937) \\ \text { N } & 1003 & 10880 & 23137 & 4372 & 10265 & 4010 & 13478 & 18772 & 13966 & 22209\end{array}$

Note: Les symboles *,** et *** correspondent respectivement aux seuils de significativité statistique de 10 pour cent, 5 pour cent et 1 pour cent. Estimations reposant sur les valeurs estimées de la relation entre santé/cognition et travail obtenue lors de la première étape avec le modèle à variables instrumentales, voir tableau 5. Les valeurs $p$ sont indiquées entre parenthèses. Les erreurs type sous-jacentes ont été estimées au moyen de la méthode bootstrap (100 itérations). C - correspond aux équations (3) à (5) présentées dans la partie 3. Le travail total (WORK) résulte de la combinaison du nombre d'heures travaillées (HOURS) et de l'emploi (EMPL).

Source: Calculs réalisés par l'auteur sur la base des données issues de SHARE (2004-2017). 
que 2,48 points de pourcentage de la diminution observée du nombre hebdomadaire d'heures de travail. D'après les erreurs type obtenues par la méthode bootstrap, cette proportion n'est pas statistiquement différente de zéro. Pour ce qui est de l'emploi, la santé ou la cognition expliquent 4,76 points de pourcentage de la diminution observée, soit une proportion statistiquement significative. Enfin, la part de la diminution du travail total expliquée par la santé ou la cognition s'établit à environ 6,61 points de pourcentage. Dans les autres pays, les proportions sont toujours statistiquement significatives pour le taux d'emploi, atteignant 35,5 pour cent dans le cas de la Pologne. Le Luxembourg est le seul pays où cette proportion n'est pas statistiquement différente de zéro.

La figure 6 est une représentation visuelle des résultats présentés dans le tableau 6, qu'elle enrichit en présentant la capacité de travail prédite à tous les âges possibles entre 50 et 70 ans. Elle met en évidence, apparemment surtout dans des pays comparativement riches comme l'Allemagne, l'Autriche, le Danemark, la Suède et la Suisse, une diminution relativement limitée et régulière entre 50 et 70 ans. Tous pays confondus, une réduction très faible du nombre d'heures travaillées (HOURS) est prédite, ce qui est la conséquence directe de l'impact très limité - si tant est qu'il existe - de l'altération de la santé et de la cognition sur le nombre d'heures travaillées au sein de la population de 50 à 54 ans, tel qu'il ressort de la première étape. De plus, les prédictions consistent essentiellement en une diminution du taux d'emploi, ce qui est également cohérent avec les résultats de la première étape, et, en toute logique, la prédiction de la diminution de l'offre de travail total coïncide presque parfaitement avec la diminution de la marge extensive.

Les figures 7, 8 et 9 sont des représentations graphiques des résultats présentés dans le tableau 6, partie C. Elles comparent la capacité de travail prédite au travail effectivement observé chez les personnes de plus de 50 ans. Elles montrent systématiquement, dans tous les pays, qu'il existe un écart important entre la capacité de travail (prédite par l'évolution de la santé ou de la cognition) et le travail effectif. En Suède, le travail total (WORK) prédit passe de 37,8 à 34 heures entre 50 et 70 ans (figure 7). À l'inverse, le travail effectif à 70 ans est proche de zéro. L'écart est moins spectaculaire à 65 ou 60 ans, mais il reste très grand. Il est permis d'en déduire que, même à ces âges, il existe une importante capacité de travail inutilisée. Le travail observé coïncide (visuellement) avec le travail prédit compte tenu de la santé et de la cognition seulement avant 60 ans. La figure 7 révèle en outre une disparité entre les pays européens en ce qui concerne l'âge auquel un écart important apparaît entre la capacité de travail estimée et le travail réel. La Suède est le pays où cet écart apparaît le plus tard, ce qui s'explique par les performances bien connues du pays en matière de maintien dans l'emploi des seniors (Martin, 2018).

Pour terminer, arrêtons-nous sur le lien entre la capacité de travail prédite à 70 ans et la prospérité économique des pays étudiés ${ }^{11}$. Bien que ces pays soient tous européens ${ }^{12}$ et soient donc relativement similaires si l'on se place d'un point

11 Pour les besoins de cette analyse, nous utilisons des données du tableau Penn World, version 9.1 (2020) (https://www.rug.nl/ggdc/productivity/pwt/pwt-releases/pwt9.1). Voir Feenstra, Inklaar et Timmer (2015).

12 Hormis Israël. 

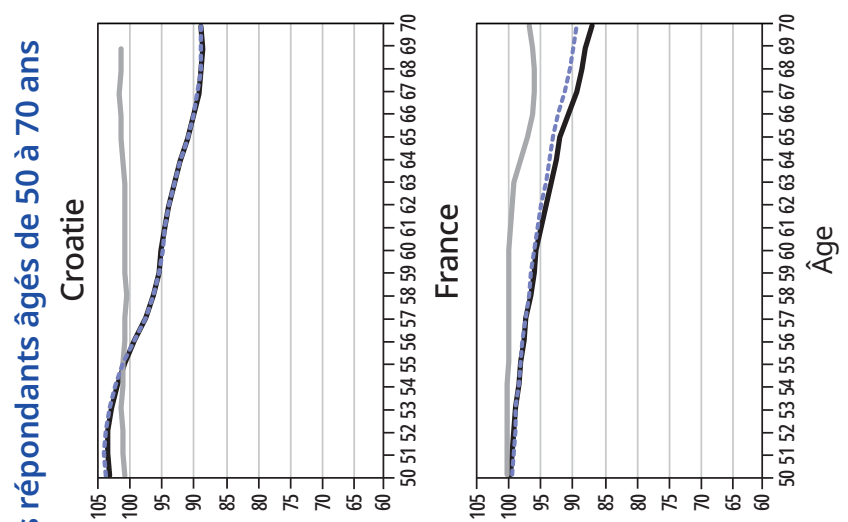

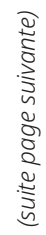
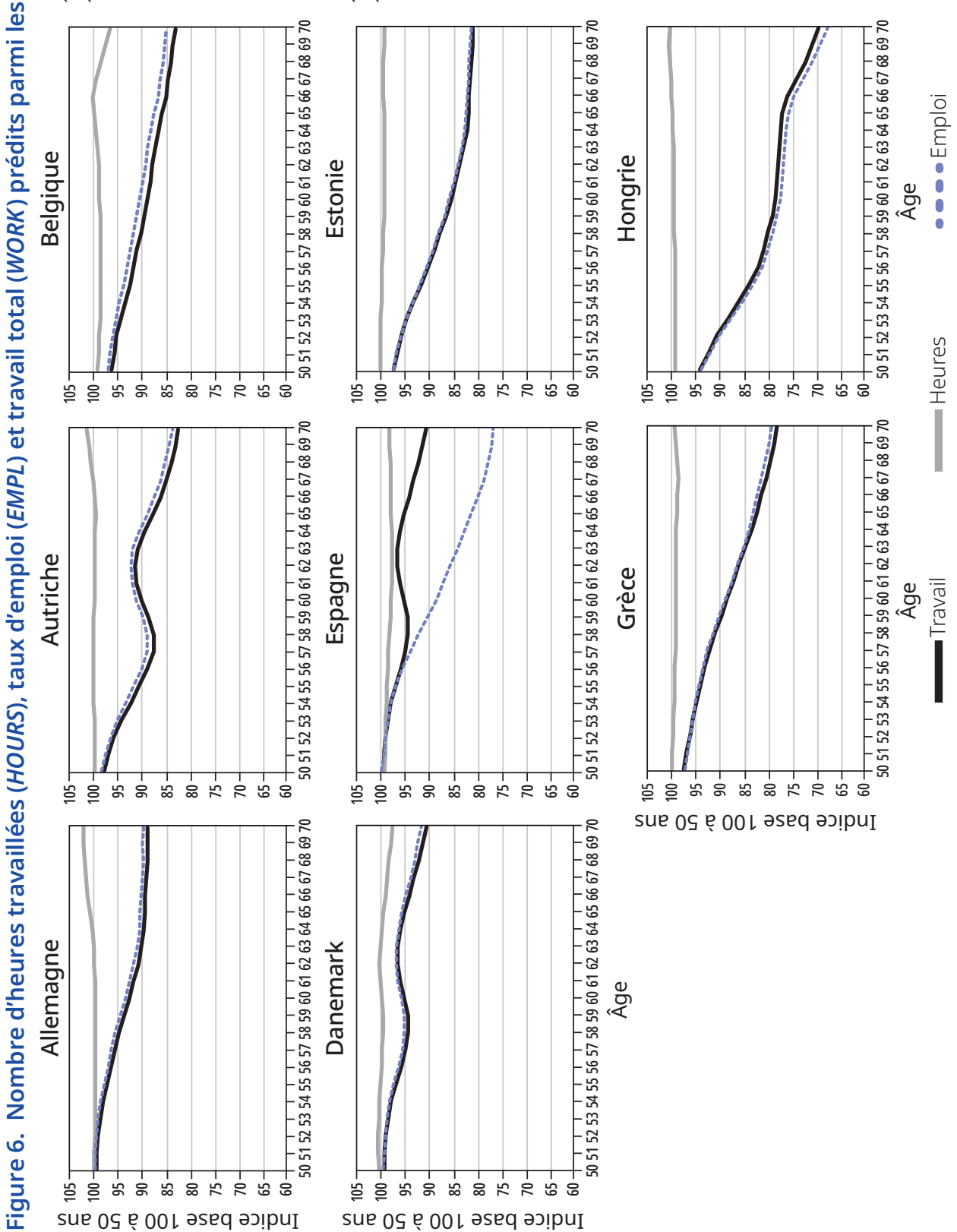

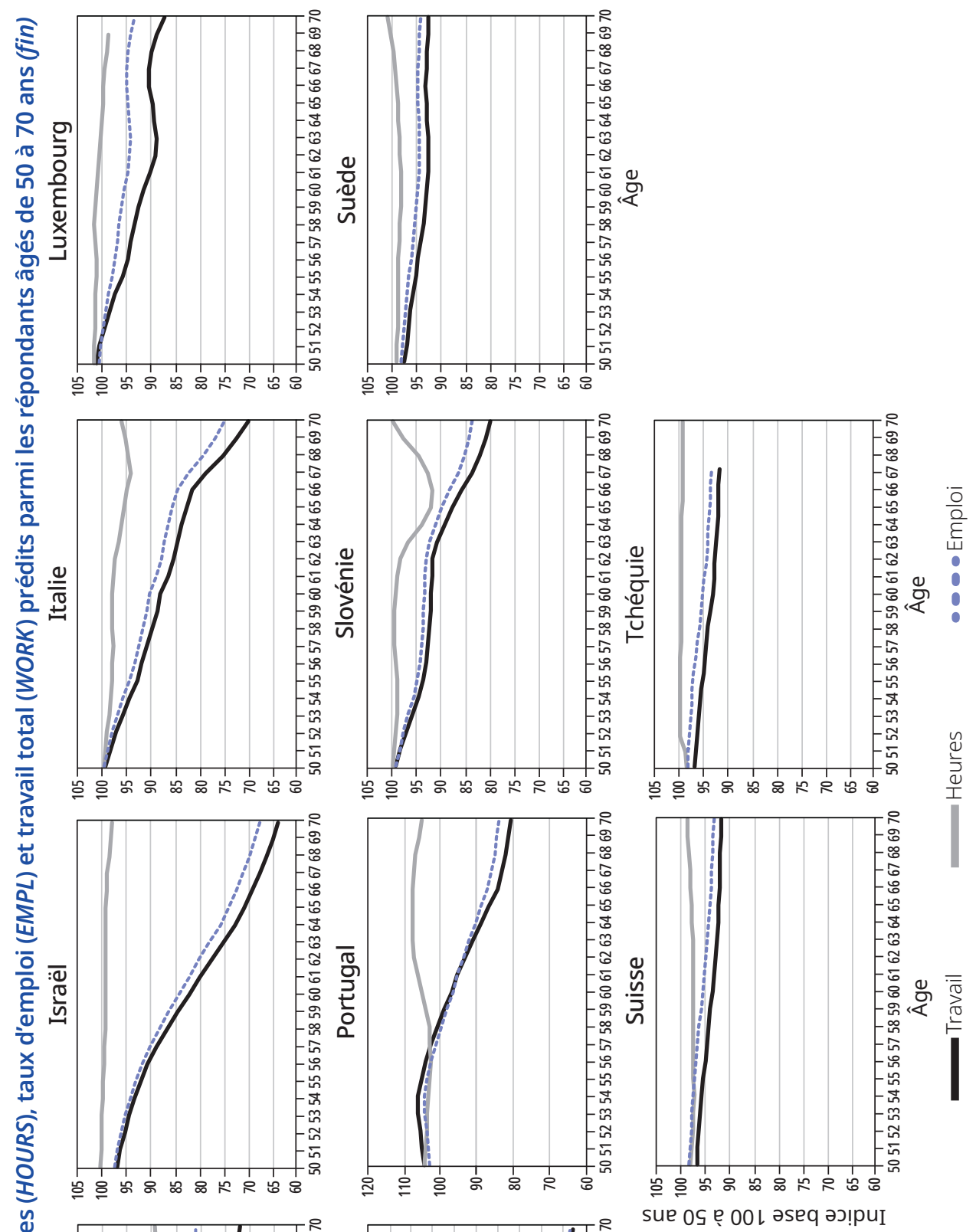

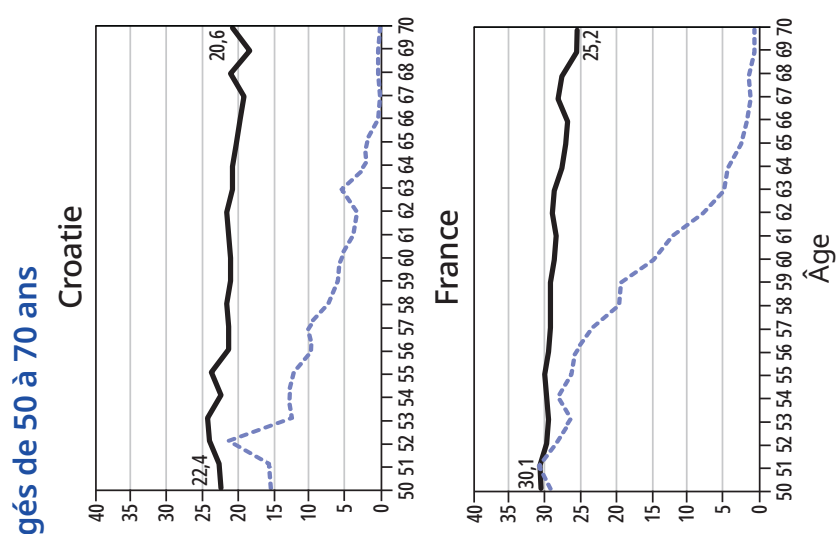

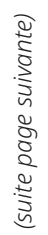
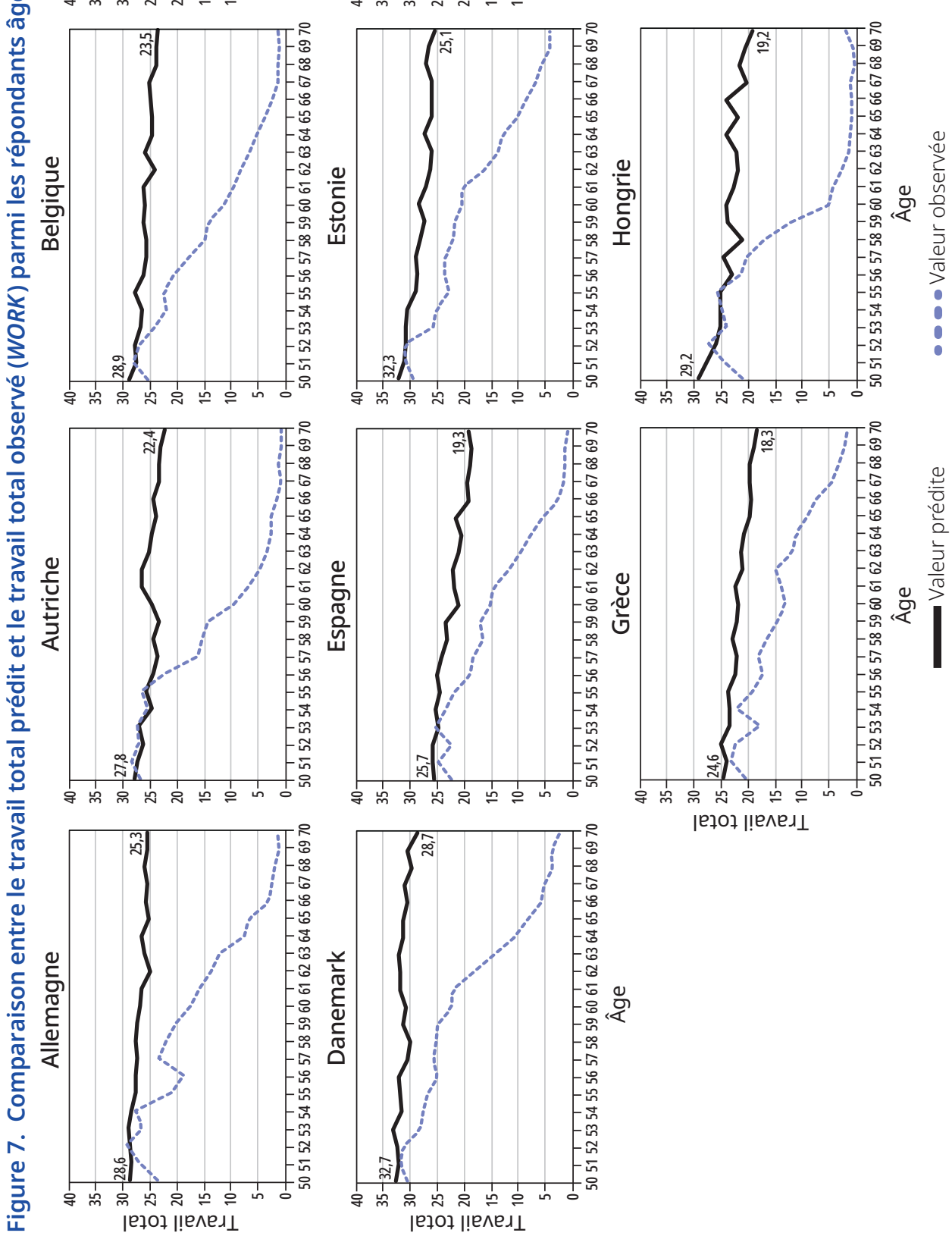

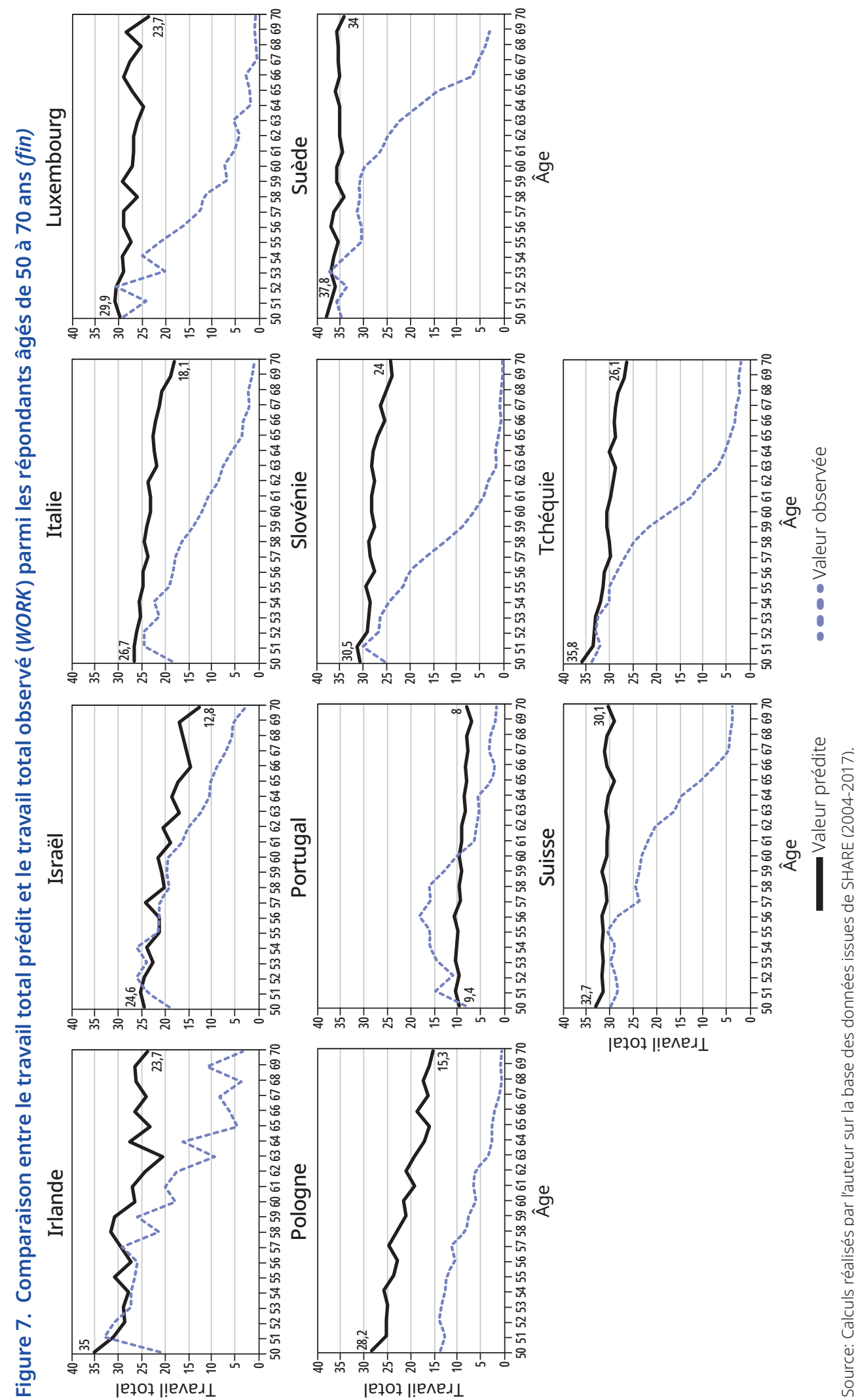

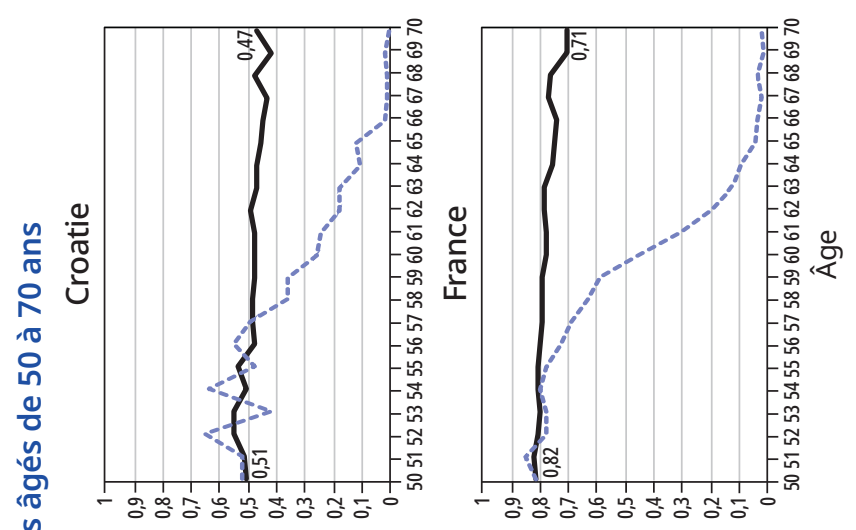

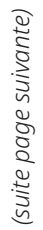
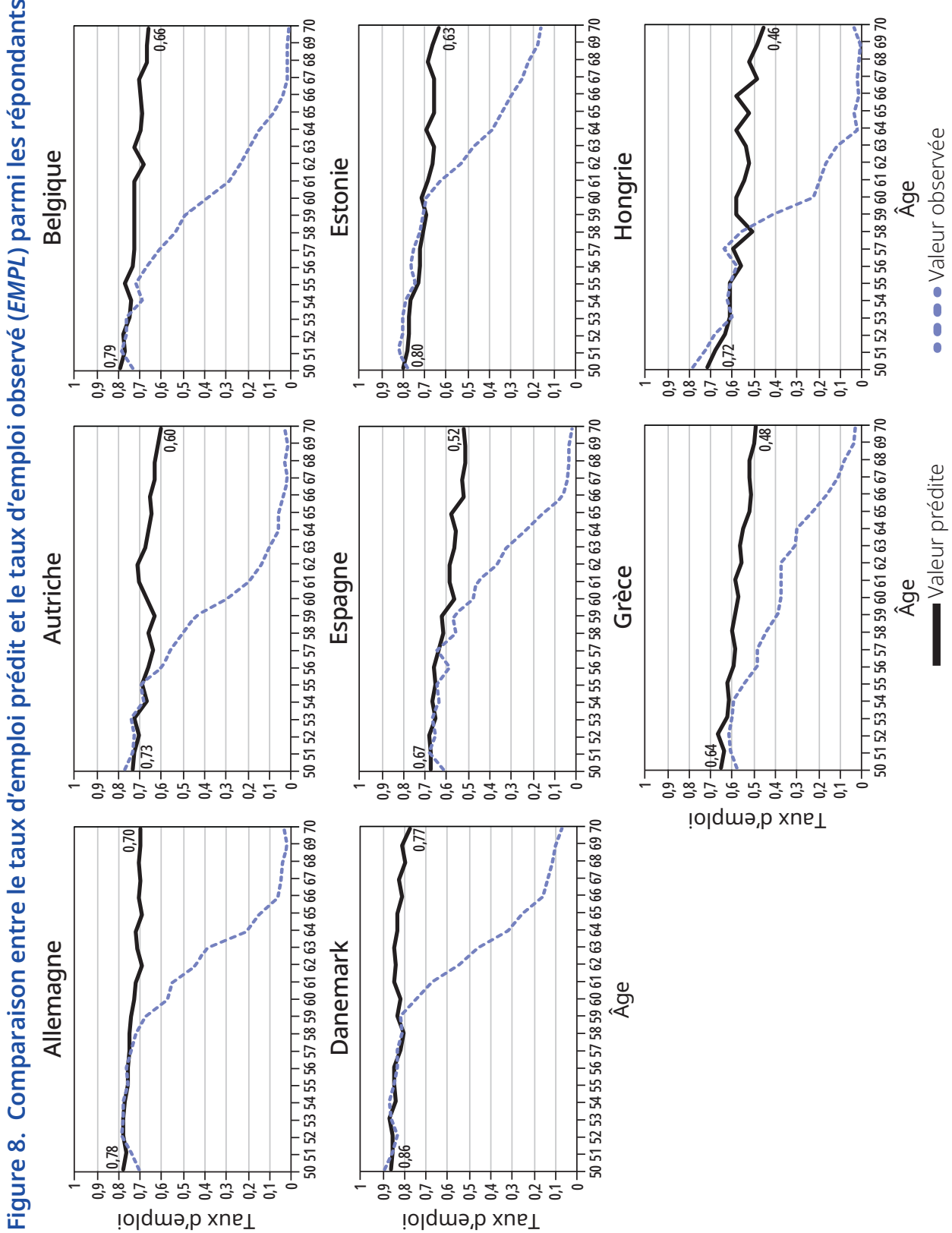

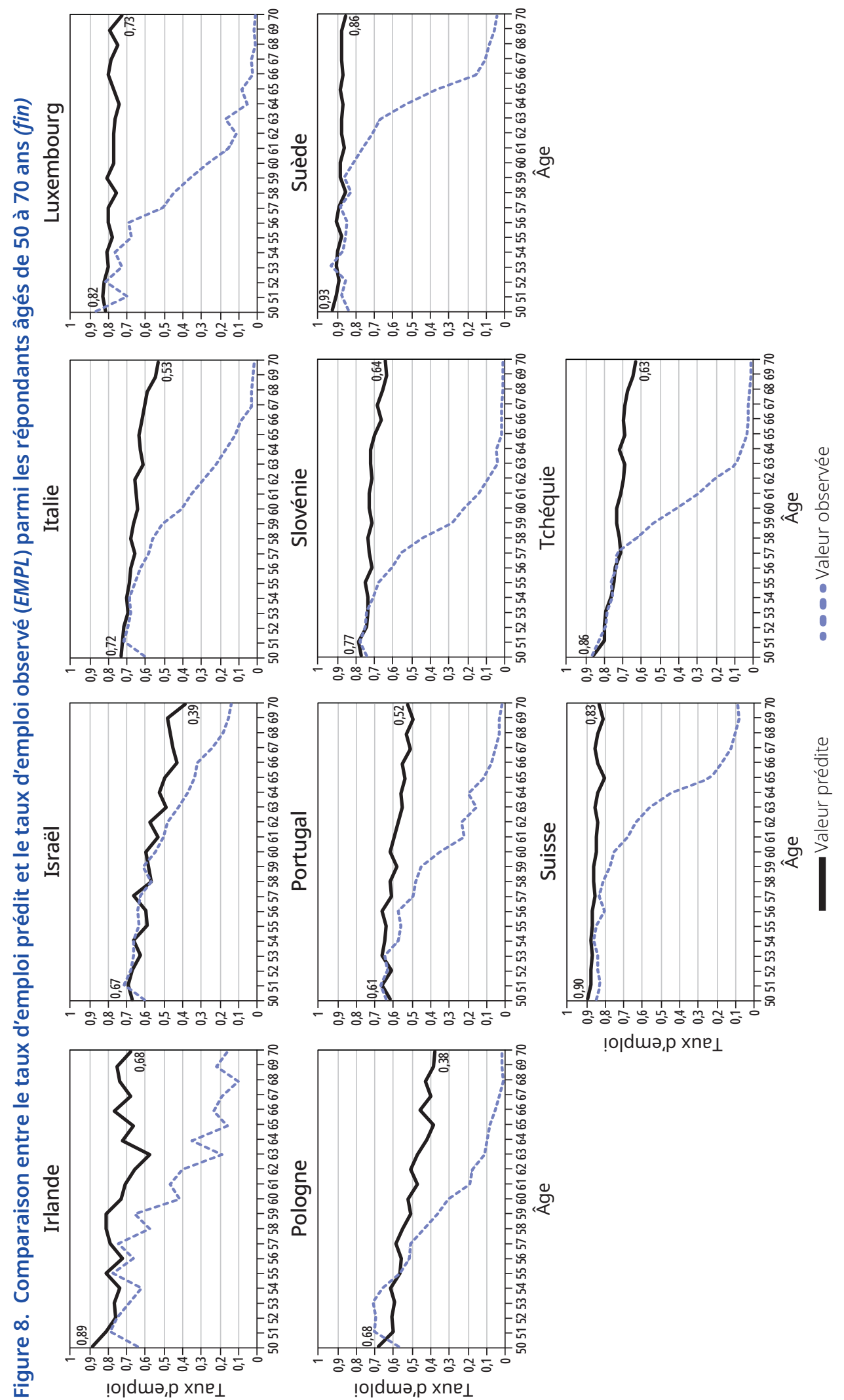

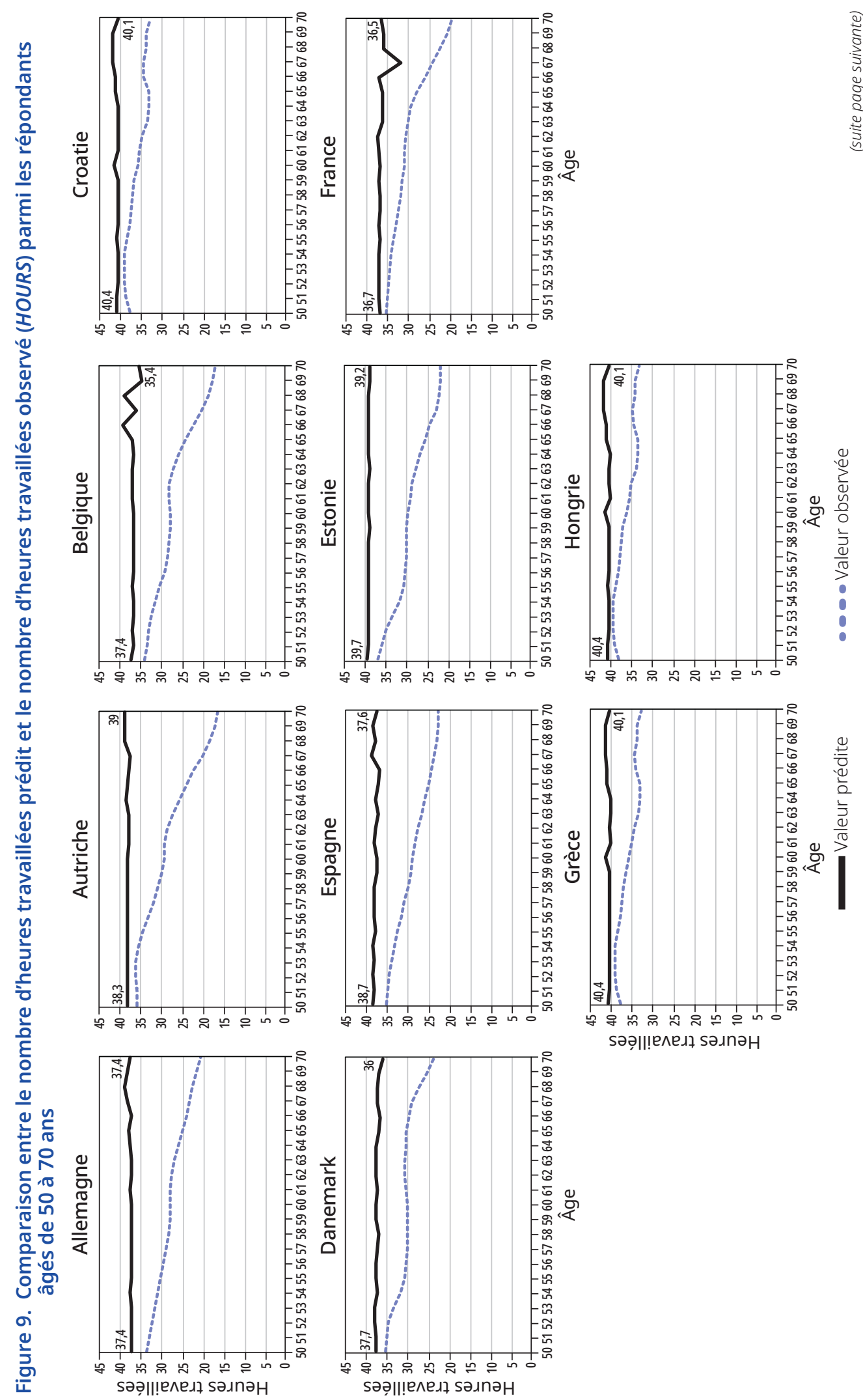

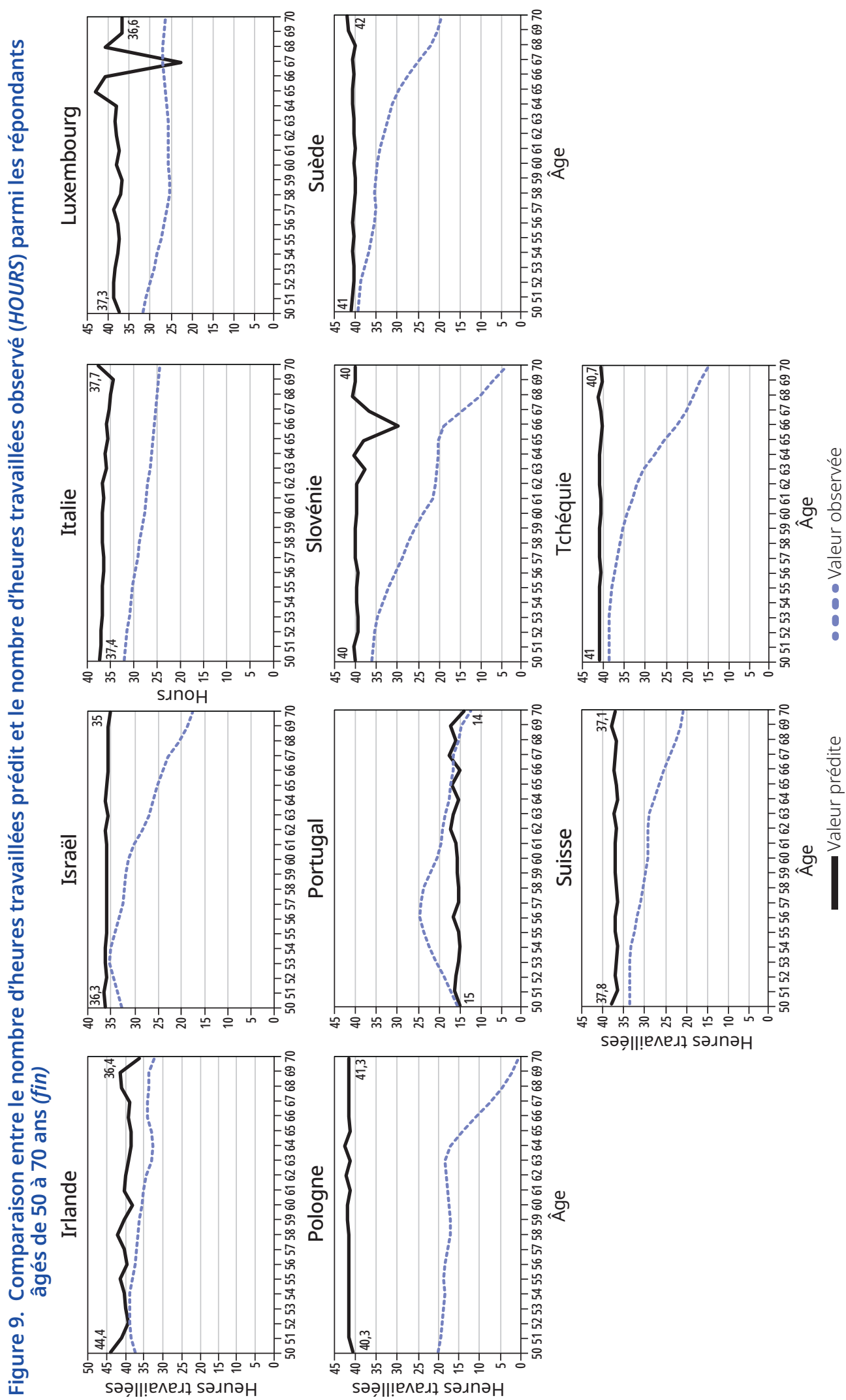
de vue mondial, ils diffèrent sensiblement en matière de conditions de vie relatives. Ainsi, le produit intérieur brut (PIB) par habitant de la Suisse est plus de deux fois plus élevé que celui de la Pologne, et nettement supérieur à celui de la Belgique. Ces différences jouent-elles un rôle dans la capacité de travail des plus de 50 ans? Un graphique simple (figure 10) laisse penser qu'on peut répondre par l'affirmative. Il existe une corrélation positive entre le PIB par habitant et la capacité de travail prédite à 70 ans, en particulier le taux d'emploi prédit (EMPL). Il s'agit d'une corrélation simple, qui ne doit pas être interprétée comme indiquant une causalité, mais elle laisse penser qu'il existe un lien relativement fort entre le PIB par habitant et la santé moyenne de la population et sa dégradation avec l'âge.

Figure 10. Capacité de travail prédite à 70 ans et PIB par habitant (taux d'emploi prédit (EMPL) et travail total (WORK))

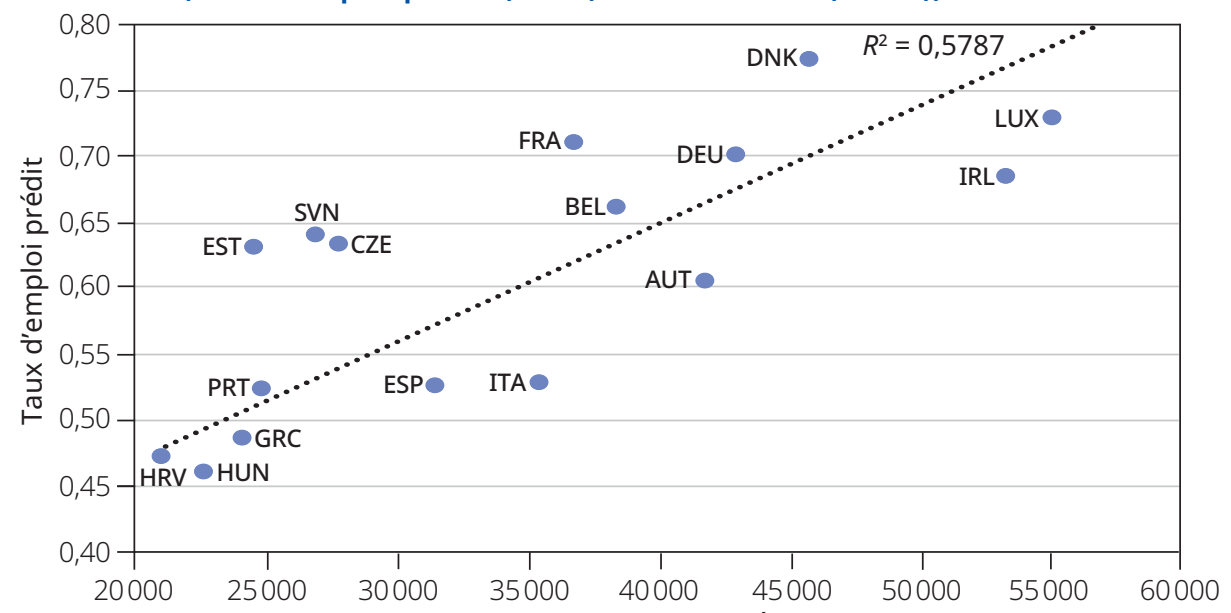

PIB par habitant (en dollars É.-U. de 2011)

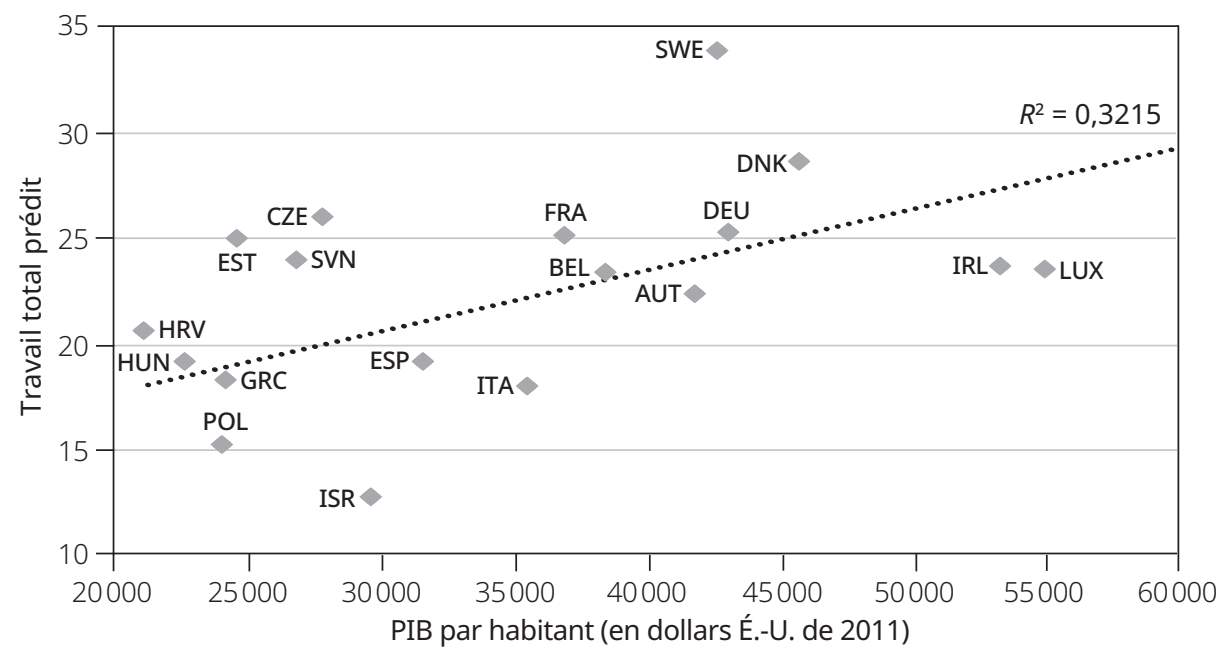

Note: Les pays sont désignés par leur code ISO 3166-1 alpha-3.

Source: Calculs réalisés par l'auteur sur la base des données issues de SHARE (2004-2017) et du tableau Penn World, version 9.1 (certains pays, qui ne sont pas couverts par les deux séries de données, n'apparaissent pas sur cette figure). 


\section{Conclusion}

L’augmentation du taux de dépendance des personnes âgées en Europe et ailleurs explique la multiplication des réformes visant à induire une hausse de l'âge effectif de la retraite. Reste cependant une question récurrente: l'état de santé et les capacités cognitives des seniors leur permettent-ils de travailler plus longtemps? Dans cet article, nous avons exploré cette question en tentant de déterminer le travail que pourraient (en moyenne) fournir les seniors s'ils étaient caractérisés par une relation entre santé ou cognition et travail identique à celle observée chez les personnes de 50 à 54 ans. La méthode employée repose sur l'hypothèse selon laquelle l'impact éventuel de la santé et/ou de la cognition sur la capacité de travail ne varie pas avec l'âge: on suppose donc que l'effet négatif de l'altération de la santé ou de la cognition observé chez les sujets de 50 à 54 ans est un prédicteur valide de l'effet de cette altération à tous les âges compris entre 55 et 70 ans. Qui plus est, on suppose que le travail lui-même (et son accumulation) n’a pas d'incidence sur la santé ou la cognition. Il s'agit là d'une limite potentiellement importante, à laquelle certains économistes se sont intéressés - voir Bassanini et Caroli (2015) pour une revue des conclusions (plutôt contrastées) des études réalisées ${ }^{13}$. Il importe de garder ces limites à l'esprit lorsque l'on interprète les résultats présentés dans cet article.

L'article constitue cependant une contribution intéressante pour plusieurs raisons. Étant donné qu'il repose sur les microdonnées totalement harmonisées et comparables de l'enquête SHARE, il a une forte dimension internationale, évaluant la capacité de travail dans vingt pays qui diffèrent les uns des autres à de multiples égards (PIB par habitant, institutions de la protection sociale et du marché du travail). Nous mesurons non seulement la santé physique, mais aussi la cognition. De plus, nous n'évaluons pas seulement la santé subjective, mais prenons aussi en compte de nombreux items «objectifs», dont des pathologies mesurables et ayant fait l'objet d'un diagnostic médical. Quant au travail, il est examiné à la marge extensive (emploi) comme à la marge intensive (nombre d'heures de travail). Autre intérêt évident de l'article: l'utilisation faite de l'économétrie pour éliminer un certain nombre de biais, en particulier pour réaliser une estimation fiable de la relation entre santé et travail chez les personnes âgées de 50 à 54 ans. Il montre que la méthode des MCO telle qu'employée par Wise (2017) conduit à sous-estimer l'impact négatif de l'altération de la santé sur la capacité de travail.

Les résultats recueillis dans cet article permettent de tirer cinq grandes conclusions. Premièrement, il existe de bonnes raisons de penser que les personnes de 50 à 54 ans qui souffrent de problèmes de santé réduisent sensiblement leur participation au marché du travail (marge extensive). Ce résultat n’est guère surprenant et confirme les constatations d’études réalisées par d’autres

13 Certains auteurs constatent que le travail, en particulier de longues journées de travail ou le travail de nuit, accélère la dégradation de la santé, tandis que d'autres observent exactement l'inverse. De nombreuses études ont été menées pour déterminer si la retraite avait un effet positif sur la santé. De même, de nombreux travaux ont été consacrés à la question de savoir si la retraite est bonne ou mauvaise pour la cognition (Mazzonna et Peracchi, 2012; Bonsang, Adam et Perelman, 2012). 
économistes au moyen de la méthode de Cutler (Wise, 2017; Coile, Milligan et Wise, 2016; Banks, Emmerson et Tetlow, 2016; Cutler, Meara et Richards-Shubik, 2013).

Deuxièmement, nos résultats montrent également que la dégradation de la santé a aussi un effet négatif sur le nombre d'heures travaillées, mais que cet effet est de nettement moindre ampleur et n'est en général pas significatif statistiquement. Il est permis d'en déduire que, à tout le moins en Europe, les seniors qui ont des problèmes de santé procèdent rarement à un ajustement du travail par la marge intensive, arrêtant plutôt de travailler.

Troisièmement, contrairement à l'altération de la santé, l'altération de la cognition n'a pas d'incidence sur le travail chez les personnes de 50 à 54 ans. En conséquence, la cognition et sa diminution sous l'effet de l'âge n'ont pas vraiment de pouvoir explicatif supplémentaire pour la prédiction de la capacité de travail $^{14}$. Ce résultat va dans le même sens que les constatations récentes de Blundell et ses coauteurs (2017) pour le Royaume-Uni et les États-Unis.

Quatrièmement, rien ne démontre que les données autodéclarées sur les obstacles au travail liés à la santé soient faussées par un «biais de justification» tel qu'envisagé par Bound (1991). Nous constatons en réalité l'inverse lorsque nous instrumentons la santé autodéclarée (en faisant appel à un modèle à variables instrumentales) au moyen des indicateurs objectifs issus de l'enquête SHARE, notamment les affections diagnostiquées par un médecin et les tests physiques et de dextérité conduits par les enquêteurs. Nous avançons que ce résultat plaide plutôt en faveur de l'existence d'une erreur de mesure plus grande, connue pour induire un biais à la baisse.

Cinquièmement, la dégradation de la santé et/ou de la cognition explique au plus 35 pour cent de la réduction du travail observée entre 50 et 70 ans - et un pourcentage plus faible parmi les seniors légèrement plus jeunes. Ce résultat semble montrer que, au sein d'un échantillon relativement vaste et varié de pays européens, beaucoup de seniors ont la capacité de travailler jusqu'à 70 ans. Cette constatation confirme celle de Wise (2017), que nous enrichissons en mettant en évidence l'existence d'une corrélation positive entre le PIB par habitant et la capacité de travail à 70 ans.

Si la capacité de travail déterminée par la santé ou la cognition demeure intrinsèquement élevée, sans doute les décideurs publics qui cherchent à augmenter le taux d'emploi des seniors devraient-ils s'intéresser aux autres déterminants de l'emploi pour cette catégorie de la population. En font partie des déterminants liés à l'offre de travail, par exemple la préférence pour les loisirs, la corrélation entre les décisions prises par les membres d'un couple en matière de retraite, l'obligation de s'occuper de proches et - bien que de nombreuses réformes des retraites aient cherché à y remédier - l'absence d'intérêt financier à différer son départ en retraite. Parmi les déterminants liés à la demande de travail (aux entreprises et employeurs) figurent une discrimination rampante liée à l'âge, qui pourrait faire sérieusement obstacle à la capacité de travail des

14 On pourrait cependant aussi voir dans ce résultat le signe que les données issues de l'enquête SHARE mesurent mieux la santé physique que la cognition, en tout cas s'agissant des dimensions qui influent sur le travail. 
seniors (Neumark, Burn et Button, 2017), et une moindre employabilité due à un rapport productivité sur coûts salariaux faible en comparaison des travailleurs jeunes ou d’âge très actif (Dostie, 2011; Vandenberghe, 2011 et 2013; van Ours et Stoeldraijer, 2011).

Soulignons également que ces conclusions ne doivent pas conduire à penser que toutes les personnes dont l'état de santé ou les capacités cognitives sont compatibles avec l'exercice d'une activité professionnelle doivent impérativement travailler. Certaines personnes peuvent parfaitement préférer prendre leur retraite plus tôt en percevant une pension plus faible et certains pays ont peutêtre les moyens ou la volonté de permettre à la population de prendre sa retraite avant que sa capacité de travail ne diminue. Au demeurant, dans leur majorité, les régimes de retraite des pays d'Europe n'ont pas (ou plus) pour vocation première de fonctionner comme des régimes d'invalidité ${ }^{15}$.

Enfin, rappelons que les méthodes et résultats présentés dans cet article portent sur la capacité de travail de la population en général. Même si, comme nous le montrons clairement, la plupart des individus ont une santé et des capacités cognitives suffisantes pour travailler jusqu'à 70 ans, de nombreuses personnes sont en trop mauvaise santé pour travailler, dans certains cas dès 50 à 54 ans $^{16}$. Ainsi, comme l'ont déjà fait observer Coile, Milligan et Wise (2016) et Wise (2017), il est indispensable que les responsables de l'action publique tiennent compte des besoins de ces personnes, par exemple à travers des régimes d'assurance-invalidité et des politiques actives du marché du travail bien conçus, permettant de déterminer de manière fiable si la capacité de travail est limitée voire inexistante.

\section{Références}

Acemoglu, Daron. 2010. «When Does Labor Scarcity Encourage Innovation?», Journal of Political Economy, 118 (6): 1037-1078.

—, et Pascual Restrepo. 2018. «Demographics and Automation», NBER Working Paper No. 24421. Cambridge (États-Unis): National Bureau of Economic Research.

Anger, Silke, et Guido Heineck. 2010. «Cognitive Abilities and Earnings: First Evidence for Germany", Applied Economics Letters, 17 (7): 699-702.

Atalay, Kadir, et Garry F. Barrett. 2015. «The Impact of Age Pension Eligibility Age on Retirement and Program Dependence: Evidence from an Australian Experiment», Review of Economics and Statistics, 97 (1): 71-87.

Baker, Michael, Mark Stabile et Catherine Deri. 2004. «What Do Self-Reported, Objective, Measures of Health Measure?», Journal of Human Resources, 39 (4): 1067-1093.

Banks, James, Carl Emmerson et Gemma Tetlow. 2016. «Health Capacity to Work at Older Ages: Evidence from the United Kingdom», NBER Working Paper No. 21980. Cambridge (États-Unis): National Bureau of Economic Research.

Bassanini, Andrea, et Eve Caroli. 2015. «Is Work Bad for Health? The Role of Constraint versus Choice», Annals of Economics and Statistics, ${ }^{\circ}$ 119/120, 13-37.

Blöndal, Sveinbjörn, et Stefano Scarpetta. 1999. "The Retirement Decision in OECD Countries», documents de travail du Département des affaires économiques de l'OCDE, $\mathrm{n}^{\circ}$ 202. Paris: OCDE.

15 Pour une analyse approfondie de l'évolution sur une longue période du rôle des régimes de retraite aux États-Unis, voir Costa (1998, pp. 6-31).

16 S'il en allait autrement, il ne serait pas possible d'estimer le lien entre santé et travail et de calculer des estimations de la capacité de travail. 
Blundell, Richard, Jack Britton, Monica Costa Dias et Eric French. 2017. «The Impact of Health on Labour Supply near Retirement», IFS Working Paper W17/18. Londres: Institute for Fiscal Studies.

Bonsang, Eric, Stéphane Adam et Sergio Perelman. 2012. «Does Retirement Affect Cognitive Functioning?», Journal of Health Economics, 31 (3): 490-501.

Börsch-Supan, Axel. 2014. "Aging Societies: Individual and Societal Plasticity», MEA Discussion Paper No. 201422. Munich: Munich Center for the Economics of Aging.

-, Martina Brandt, Christian Hunkler, Thorsten Kneip, Julie Korbmacher, Frederic Malter, Barbara Schaan, Stephanie Stuck et Sabrina Zuber. 2013. «Data Resource Profile: The Survey of Health, Ageing and Retirement in Europe (SHARE)», International Journal of Epidemiology, 42 (4): 992-1001.

Bound, John. 1991. «Self-Reported versus Objective Measures of Health in Retirement Models», Journal of Human Resources, 26 (1): 106-138.

Chang, Angela Y., Vegard F. Skirbekk, Stefanos Tyrovolas, Nicholas J. Kassebaum et Joseph L. Dieleman. 2019. "Measuring Population Ageing: An Analysis of the Global Burden of Disease Study 2017», Lancet Public Health, 4 (3): E159-E167.

Coile, Courtney, Kevin S. Milligan et David A. Wise. 2016. «Health Capacity to Work at Older Ages: Evidence from the U.S.», NBER Working Paper No. 21940. Cambridge (États-Unis): National Bureau of Economic Research.

Costa, Dora L. 1998. The Evolution of Retirement: An American Economic History, 18801990. Chicago: University of Chicago Press.

Cutler, David M., Kaushik Ghosh et Mary Beth Landrum. 2013. «Evidence for Significant Compression of Morbidity in the Elderly U. S. Population», NBER Working Paper No. 19268. Cambridge (États-Unis): National Bureau of Economic Research.

—, Ellen Meara et Seth Richards-Shubik. 2013. «Health and Work Capacity of Older Adults: Estimates and Implications for Social Security Policy», SSRN Working Paper. https://dx.doi.org/10.2139/ssrn.2577858.

Delmez, Françoise, et Vincent Vandenberghe. 2018. «Long Working Hours Make Us Less Productive but Also Less Costly", Labour, 32 (4): 259-287.

De Souza, Laeticia R., Bernardo L. Queiroz et Vegard F. Skirbekk. 2019. «Trends in Health and Retirement in Latin America: Are Older Workers Healthy Enough to Extend their Working Lives?», Journal of the Economics of Ageing, 13 (mai): 72-83.

Dorn, David, et Alfonso Sousa-Poza. 2010. "“Voluntary” and "Involuntary” Early Retirement: An International Analysis», Applied Economics, 42 (4): 427-438.

Dostie, Benoit. 2011. «Wages, Productivity and Aging», De Economist, 159 (2): 139-158.

Dwyer, Debra Sabatini, et Olivia S. Mitchell. 1999. «Health Problems as Determinants of Retirement: Are Self-Rated Measures Endogenous?», Journal of Health Economics, 18 (2): 173-193.

Feenstra, Robert C., Robert Inklaar et Marcel P. Timmer. 2015. «The Next Generation of the Penn World Table», American Economic Review, 105 (10): 3150-3182.

Hutchens, Robert. 1986. «Delayed Payment Contracts and a Firm's Propensity to Hire Older Workers», Journal of Labor Economics, 4 (4): 439-457.

-. 2010. «Worker Characteristics, Job Characteristics, and Opportunities for Phased Retirement», Labour Economics, 17 (6): 1010-1021.

Jousten, Alain, Mathieu Lefèbvre, Sergio Perelman et Pierre Pestieau. 2010. «The Effects of Early Retirement on Youth Unemployment: The Case of Belgium», dans Social Security Programs and Retirement around the World: The Relationship to Youth Employment, publ. sous la dir. de Jonathan Gruber et David A. Wise, 47-76. Chicago: University of Chicago Press.

Martin, John P. 2018. Live Longer, Work Longer: The Changing Nature of the Labour Market for Older Workers in OECD Countries, IZA Discussion Paper No. 11510. Bonn: Institute of Labor Economics. 
Mazzonna, Fabrizio, et Franco Peracchi. 2012. «Ageing, Cognitive Abilities and Retirement», European Economic Review, 56 (4): 691-710.

Michaud, Pierre-Carl, Arthur Van Soest, et Luc Bissonnette. 2020. «Understanding Joint Retirement», Journal of Economic Behavior \& Organization, 173 (mai): 386-401.

Milligan, Kevin S., et David A. Wise. 2012. «Health and Work at Older Ages: Using Mortality to Assess the Capacity to Work across Countries», NBER Working Paper No. 18229. Cambridge (États-Unis): National Bureau of Economic Research.

Moran, John R., Pamela Farley Short et Christopher S. Hollenbeak. 2011. «Long-Term Employment Effects of Surviving Cancer», Journal of Health Economics, 30 (3): 505-514.

Neumark, David, Ian Burn et Patrick Button. 2017. «Is It Harder for Older Workers to Find Jobs? New and Improved Evidence from a Field Experiment», NBER Working Paper No. 21669 (révisé). Cambridge (États-Unis): National Bureau of Economic Research.

OCDE (Organisation de coopération et de développement économiques) et Union européenne. 2016. Health at a Glance: Europe 2016 - State of Health in the EU Cycle. Paris.

O'Donnell, Owen, Eddy Van Doorslaer et Tom Van Ourti. 2015. «Health and Inequality», dans Handbook of Income Distribution, publ. sous la dir. d'Anthony B. Atkinson et François Bourguignon, vol. 2B, 1419-1533. Amsterdam: North Holland.

Oeppen, Jim, et James W. Vaupel. 2002. «Broken Limits to Life Expectancy», Science, 296 (5570): 1029-1031.

Oliveira Martins, Joaquim, Frédéric Gonand, Pablo Antolín, Christine de la Maisonneuve et Kwang-Yeol Yoo. 2005. «The Impact of Ageing on Demand, Factor Markets and Growth», documents de travail du Département des affaires économiques de l'OCDE, $\mathrm{n}^{\circ}$ 420. Paris: OCDE.

Pelkowski, Jodi Messer, et Mark C. Berger. 2004. «The Impact of Health on Employment, Wages, and Hours Worked over the Life Cycle», Quarterly Review of Economics and Finance, 44 (1): 102-121.

Salthouse, Timothy A. 2010. "Selective Review of Cognitive Aging», Journal of the International Neuropsychological Society, 16 (5): 754-760.

Skirbekk, Vegard. 2004. «Age and Individual Productivity: A Literature Survey», Vienna Yearbook of Population Research, 2: 133-154.

Stern, Steven. 1989. «Measuring the Effect of Disability on Labor Force Participation», Journal of Human Resources, 24 (3): 361-395.

Vandenberghe, Vincent. 2011. «Boosting the Employment Rate of Older Men and Women», De Economist, 159 (2): 159-191.

—. 2013. «Are Firms Willing to Employ a Greying and Feminizing Workforce?», Labour Economics, 22 (juin): 30-46.

-. 2020. «Ageing Calls for Shorter Full-Time Tertiary Education and Increased Continuing Education», Discussion Paper 2020/01. Louvain-la-Neuve: IRES-LIDAM.

—, Fábio Waltenberg et Mariann Rigó. 2013. «Ageing and Employability: Evidence from Belgian Firm-Level Data», Journal of Productivity Analysis, 40 (1): 111-136.

van Ours, Jan C., et Lenny Stoeldraijer. 2011. «Age, Wage and Productivity in Dutch Manufacturing», De Economist, 159 (2): 113-137.

Wise, David A. (dir.). 2017. Social Security Programs and Retirement around the World: The Capacity to Work at Older Ages. Chicago: University of Chicago Press. 


\section{Annexe}

Tableau A1. Résultats de la première étape (MCO): impact de l'altération de l'état de santé et de la cognition sur le travail parmi les répondants de 50 à 54 ans

\begin{tabular}{|c|c|c|c|c|c|c|c|c|c|c|}
\hline & Allemagne & Autriche & Belgique & Croatie & Danemark & Espagne & Estonie & France & Grèce & Hongrie \\
\hline & \multicolumn{10}{|c|}{ A. Indicateur d'altération de l'état de santé } \\
\hline$\beta_{h}^{\text {WORK }}$ & $\begin{array}{l}-6,30^{* * *} \\
(0,000)\end{array}$ & $\begin{array}{l}-7,56^{* * *} \\
(0,000)\end{array}$ & $\begin{array}{l}-5,95 * * * \\
(0,000)\end{array}$ & $\begin{array}{l}-6,56^{* * *} \\
(0,000)\end{array}$ & $\begin{array}{l}-7,66^{* * *} \\
(0,000)\end{array}$ & $\begin{array}{l}-5,99^{* * *} \\
(0,000)\end{array}$ & $\begin{array}{l}-6,80^{* * *} \\
(0,000)\end{array}$ & $\begin{array}{l}-5,24^{* * *} \\
(0,000)\end{array}$ & $\begin{array}{l}-3,70^{* * *} \\
(0,000)\end{array}$ & $\begin{array}{l}-8,23^{* * * *} \\
(0,000)\end{array}$ \\
\hline$\beta_{h}^{E M P L}$ & $\begin{array}{l}-0,16^{* * *} \\
(0,000)\end{array}$ & $\begin{array}{l}-0,19^{* * *} \\
(0,000)\end{array}$ & $\begin{array}{l}-0,15^{* * *} \\
(0,000)\end{array}$ & $\begin{array}{l}-0,15^{* * *} \\
(0,000)\end{array}$ & $\begin{array}{l}-0,19^{* * *} \\
(0,000)\end{array}$ & $\begin{array}{l}-0,14^{* * *} \\
(0,000)\end{array}$ & $\begin{array}{l}-0,17^{* * *} \\
(0,000)\end{array}$ & $\begin{array}{l}-0,15^{* * *} \\
(0,000)\end{array}$ & $\begin{array}{l}-0,07^{* * *} \\
(0,000)\end{array}$ & $\begin{array}{l}-0,20 \text { *** } \\
(0,000)\end{array}$ \\
\hline \multirow[t]{2}{*}{$\beta_{h}^{\text {HOURS }}$} & $\begin{array}{l}-0,62 \\
(0,190)\end{array}$ & $\begin{array}{l}-0,35 \\
(0,784)\end{array}$ & $\begin{array}{l}-0,76 \\
(0,134)\end{array}$ & $\begin{array}{l}-1,15 \\
(0,144)\end{array}$ & $\begin{array}{l}-1,45^{*} \\
(0,030)\end{array}$ & $\begin{array}{l}-2,13 \\
(0,105)\end{array}$ & $\begin{array}{l}-0,21 \\
(0,655)\end{array}$ & $\begin{array}{l}-0,27 \\
(0,469)\end{array}$ & $\begin{array}{l}-1,86 \\
(0,082)\end{array}$ & $\begin{array}{l}-1,78^{* *} \\
(0,007)\end{array}$ \\
\hline & \multicolumn{10}{|c|}{ B. Indicateur d'altération de la cognition } \\
\hline$\beta_{c}^{\text {WORK }}$ & $\begin{array}{l}-0,64 \\
(0,276)\end{array}$ & $\begin{array}{l}-0,67 \\
(0,364)\end{array}$ & $\begin{array}{l}-2,49 * * * \\
(0,000)\end{array}$ & $\begin{array}{l}1,15 \\
(0,307)\end{array}$ & $\begin{array}{l}-1,68^{* *} \\
(0,007)\end{array}$ & $\begin{array}{l}-4,35^{* * *} \\
(0,000)\end{array}$ & $\begin{array}{l}-1,90^{* * *} \\
(0,000)\end{array}$ & $\begin{array}{l}-3,04^{* * *} \\
(0,000)\end{array}$ & $\begin{array}{l}0,39 \\
(0,728)\end{array}$ & $\begin{array}{l}-1,25 \\
(0,499)\end{array}$ \\
\hline$\beta_{c}^{E M P L}$ & $\begin{array}{l}-0,04^{* * *} \\
(0,001)\end{array}$ & $\begin{array}{l}-0,02 \\
(0,245)\end{array}$ & $\begin{array}{l}-0,07^{* * *} \\
(0,000)\end{array}$ & $\begin{array}{l}0,04 \\
(0,103)\end{array}$ & $\begin{array}{l}-0,03^{*} \\
(0,045)\end{array}$ & $\begin{array}{l}-0,09^{* * *} \\
(0,000)\end{array}$ & $\begin{array}{l}-0,02 \\
(0,123)\end{array}$ & $\begin{array}{l}-0,04^{* * *} \\
(0,000)\end{array}$ & $\begin{array}{l}-0,01 \\
(0,653)\end{array}$ & $\begin{array}{l}-0,05^{* * *} \\
(0,000)\end{array}$ \\
\hline$\beta_{c}^{\text {HOURS }}$ & $\begin{array}{l}0,93 \\
(0,166)\end{array}$ & $\begin{array}{l}-0,38 \\
(0,599)\end{array}$ & $\begin{array}{l}0,18 \\
(0,686)\end{array}$ & $\begin{array}{l}0,32 \\
(0,572)\end{array}$ & $\begin{array}{l}-0,39 \\
(0,380)\end{array}$ & $\begin{array}{l}-1,85^{* * *} \\
(0,000)\end{array}$ & $\begin{array}{l}-0,93^{*} \\
(0,010)\end{array}$ & $\begin{array}{l}-2,06^{* *} \\
(0,003)\end{array}$ & $\begin{array}{l}1,04 \\
(0,339)\end{array}$ & $\begin{array}{l}2,26 \\
(0,310)\end{array}$ \\
\hline \multirow[t]{3}{*}{$\mathrm{N}$} & 20680 & 18373 & 27750 & 4822 & 17206 & 24474 & 22369 & 22644 & 13710 & 4523 \\
\hline & Irlande & Israël & Italie & Luxembourg & Pologne & Portugal & Slovénie & Suède & Suisse & Tchéquie \\
\hline & \multicolumn{10}{|c|}{ A. Indicateur d'altération de l'état de santé } \\
\hline$\beta_{h}^{\text {WORK }}$ & $\begin{array}{l}-5,80^{* * *} \\
(0,001)\end{array}$ & $\begin{array}{l}-4,96^{* * *} \\
(0,000)\end{array}$ & $\begin{array}{l}-3,22 * * * \\
(0,000)\end{array}$ & $\begin{array}{l}-3,20 \\
(0,075)\end{array}$ & $\begin{array}{l}-8,60^{* * *} \\
(0,000)\end{array}$ & $\begin{array}{l}-1,60 \\
(0,142)\end{array}$ & $\begin{array}{l}-5,97^{* * *} \\
(0,000)\end{array}$ & $\begin{array}{l}-6,78^{* * *} \\
(0,000)\end{array}$ & $\begin{array}{l}-3,75^{* * *} \\
(0,000)\end{array}$ & $\begin{array}{l}-8,32^{* * *} \\
(0,000)\end{array}$ \\
\hline$\beta_{h}^{E M P L}$ & $\begin{array}{l}-0,14^{* *} \\
(0,002)\end{array}$ & $\begin{array}{l}-0,12^{* * *} \\
(0,000)\end{array}$ & $\begin{array}{l}-0,07^{* * *} \\
(0,000)\end{array}$ & $\begin{array}{l}-0,08 \\
(0,101)\end{array}$ & $\begin{array}{l}-0,20 * * * \\
(0,000)\end{array}$ & $\begin{array}{l}-0,16^{* * *} \\
(0,000)\end{array}$ & $\begin{array}{l}-0,12^{* * *} \\
(0,000)\end{array}$ & $\begin{array}{l}-0,15^{* * *} \\
(0,000)\end{array}$ & $\begin{array}{l}-0,11 * * * \\
(0,000)\end{array}$ & $\begin{array}{l}-0,21^{* * *} \\
(0,000)\end{array}$ \\
\hline \multirow[t]{2}{*}{$\beta_{h}^{\text {HOURS }}$} & $\begin{array}{l}-5,43 \\
(0,087)\end{array}$ & $\begin{array}{l}-0,46 \\
(0,648)\end{array}$ & $\begin{array}{l}-1,23^{*} \\
(0,049)\end{array}$ & $\begin{array}{l}-1,75 \\
(0,175)\end{array}$ & $\begin{array}{l}-0,25 \\
(0,728)\end{array}$ & $\begin{array}{l}2,83 \\
(0,253)\end{array}$ & $\begin{array}{l}-2,01 * \\
(0,011)\end{array}$ & $\begin{array}{l}-1,98^{* * *} \\
(0,001)\end{array}$ & $\begin{array}{l}-0,35 \\
(0,461)\end{array}$ & $\begin{array}{l}-1,03 \\
(0,165)\end{array}$ \\
\hline & \multicolumn{10}{|c|}{ B. Indicateur d'altération de la cognition } \\
\hline$\beta_{c}^{\text {WORK }}$ & $\begin{array}{l}-3,44 \\
(0,186)\end{array}$ & $\begin{array}{l}-2,09 \\
(0,250)\end{array}$ & $\begin{array}{l}-3,96^{* * *} \\
(0,000)\end{array}$ & $\begin{array}{l}-0,35 \\
(0,709)\end{array}$ & $\begin{array}{l}-0,66 \\
(0,339)\end{array}$ & $\begin{array}{l}0,10 \\
(0,961)\end{array}$ & $\begin{array}{l}-2,77^{*} \\
(0,039)\end{array}$ & $\begin{array}{l}-0,73 \\
(0,074)\end{array}$ & $\begin{array}{l}-2,41^{* *} \\
(0,002)\end{array}$ & $\begin{array}{l}-2,49 * * * \\
(0,000)\end{array}$ \\
\hline$\beta_{c}^{E M P L}$ & $\begin{array}{l}0,02 \\
(0,776)\end{array}$ & $\begin{array}{l}-0,05 \\
(0,112)\end{array}$ & $\begin{array}{l}-0,08^{* * *} \\
(0,000)\end{array}$ & $\begin{array}{l}-0,01 \\
(0,711)\end{array}$ & $\begin{array}{l}-0,04^{*} \\
(0,029)\end{array}$ & $\begin{array}{l}-0,03 \\
(0,597)\end{array}$ & $\begin{array}{l}-0,06^{*} \\
(0,019)\end{array}$ & $\begin{array}{l}0,00 \\
(0,957)\end{array}$ & $\begin{array}{l}-0,05^{* *} \\
(0,002)\end{array}$ & $\begin{array}{l}-0,04^{* *} \\
(0,004)\end{array}$ \\
\hline$\beta_{c}^{\text {HOURS }}$ & $\begin{array}{l}-5,32 * * \\
(0,006)\end{array}$ & $\begin{array}{l}-0,70 \\
(0,764)\end{array}$ & $\begin{array}{l}-2,15^{* *} \\
(0,002)\end{array}$ & $\begin{array}{l}0,78 \\
(0,408)\end{array}$ & $\begin{array}{l}1,14 \\
(0,063)\end{array}$ & $\begin{array}{l}1,58 \\
(0,602)\end{array}$ & $\begin{array}{l}-0,58 \\
(0,567)\end{array}$ & $\begin{array}{l}0,07 \\
(0,839)\end{array}$ & $\begin{array}{l}-0,33 \\
(0,709)\end{array}$ & $\begin{array}{l}-1,22 \\
(0,129)\end{array}$ \\
\hline $\mathrm{N}$ & 1003 & 10880 & 23137 & 4372 & 10265 & 4010 & 13478 & 18772 & 13966 & 22209 \\
\hline
\end{tabular}

Note: Les symboles $*,{ }^{* *}$ et ${ }^{* * *}$ correspondent respectivement aux seuils de significativité statistique de 10 pour cent, 5 pour cent et 1 pour cent. Estimations ponctuelles $\beta_{h}^{Z}$ et $\beta_{c}^{Z}$ de l'effet d'une augmentation d'un écart type de l'indicateur sur le travail total, l'emploi et le nombre d'heures travaillées. Les valeurs $p$ sont indiquées entre parenthèses. Les erreurs type sous-jacentes ont été estimées au moyen de la méthode bootstrap (100 itérations). L'indicateur de santé subjectif/physique a été instrumenté par les variables objectives figurant dans le tableau 3. Le travail total (WORK) résulte de la combinaison du nombre d'heures travaillées (HOURS) et de l'emploi (EMPL).

Source: Calculs réalisés par l'auteur sur la base des données issues de SHARE (2004-2017). 
Tableau A2. Résultats de la première étape: impact de l'état de santé physique et de la cognition sur le travail total (WORK) parmi les répondants de 50 à 54 ans et comparaison entre les résultats obtenus avec les MCO et ceux obtenus avec le modèle à variables instrumentales

\begin{tabular}{|c|c|c|c|c|c|c|c|c|c|c|}
\hline & Allemagne & Autriche & Belgique & Croatie & Danemark & Espagne & Estonie & France & Grèce & Hongrie \\
\hline & \multicolumn{10}{|c|}{ Modèle 1: indicateur d'altération de la santé et indicateur d'altération de la cognition (MCO) } \\
\hline$\beta_{h}^{\text {WORK }} M C O$ & $\begin{array}{l}-6,30^{* * *} \\
(0,000)\end{array}$ & $\begin{array}{l}-7,56^{* * *} \\
(0,000)\end{array}$ & $\begin{array}{l}-5,95^{* * *} \\
(0,000)\end{array}$ & $\begin{array}{l}-6,56^{* * *} \\
(0,000)\end{array}$ & $\begin{array}{l}-7,66^{* * *} \\
(0,000)\end{array}$ & $\begin{array}{l}-5,99 * * * \\
(0,000)\end{array}$ & $\begin{array}{l}-6,80 * * * \\
(0,000)\end{array}$ & $\begin{array}{l}-5,24 * * * \\
(0,000)\end{array}$ & $\begin{array}{l}-3,70 * * * \\
(0,000)\end{array}$ & $\begin{array}{l}-8,23 * * * \\
(0,000)\end{array}$ \\
\hline \multirow[t]{2}{*}{$\beta_{c}^{\text {WORK }} M C O$} & $\begin{array}{l}-0,64 \\
(0,350)\end{array}$ & $\begin{array}{l}-0,67 \\
(0,366)\end{array}$ & $\begin{array}{l}-2,49 * * * \\
(0,000)\end{array}$ & $\begin{array}{l}1,15 \\
(0,503)\end{array}$ & $\begin{array}{l}-1,68^{* *} \\
(0,003)\end{array}$ & $\begin{array}{l}-4,35^{* * *} \\
(0,000)\end{array}$ & $\begin{array}{l}-1,90 * * \\
(0,004)\end{array}$ & $\begin{array}{l}-3,04^{* * *} \\
(0,000)\end{array}$ & $\begin{array}{l}0,39 \\
(0,685)\end{array}$ & $\begin{array}{l}-1,25 \\
(0,442)\end{array}$ \\
\hline & \multicolumn{10}{|c|}{$\begin{array}{l}\text { Modèle 2: indicateur d'altération de la santé et indicateur d'altération de la cognition } \\
\text { (modèle à variables instrumentales) }\end{array}$} \\
\hline$\beta_{h}^{\text {WORK }} I V$ & $\begin{array}{l}-8,70 * * * \\
(0,000)\end{array}$ & $\begin{array}{l}-10,41^{* * *} \\
(0,000)\end{array}$ & $\begin{array}{l}-7,96^{* * *} \\
(0,000)\end{array}$ & $\begin{array}{l}-8,85^{* * *} \\
(0,000)\end{array}$ & $\begin{array}{l}-9,84^{* * *} \\
(0,000)\end{array}$ & $\begin{array}{l}-8,83^{* * *} \\
(0,000)\end{array}$ & $\begin{array}{l}-9,80^{* * *} \\
(0,000)\end{array}$ & $\begin{array}{l}-6,27 * * * \\
(0,000)\end{array}$ & & $\begin{array}{l}-11,44^{* * *} \\
(0,000)\end{array}$ \\
\hline \multirow[t]{2}{*}{$\beta_{c}^{\text {WORK } I V}$} & $\begin{array}{l}-0,07 \\
(0,933)\end{array}$ & $\begin{array}{l}-3,34 \\
(0,067)\end{array}$ & $\begin{array}{l}-1,57^{*} \\
(0,013)\end{array}$ & $\begin{array}{l}1,53 \\
(0,454)\end{array}$ & $\begin{array}{l}-1,00 \\
(0,528)\end{array}$ & $\begin{array}{l}-4,15^{* * *} \\
(0,000)\end{array}$ & $\begin{array}{l}-0,19 \\
(0,867)\end{array}$ & $\begin{array}{l}-3,07^{* * *} \\
(0,000)\end{array}$ & $\begin{array}{l}0,39 \\
(0,705)\end{array}$ & $\begin{array}{l}-1,15 \\
(0,568)\end{array}$ \\
\hline & \multicolumn{10}{|c|}{ Comparaison des modèles 1 et 2} \\
\hline$\beta_{h}^{\text {WORK } I V-M C O}$ & $\begin{array}{l}-2,39 * * * \\
(0,001)\end{array}$ & $\begin{array}{l}-2,85^{* *} \\
(0,007)\end{array}$ & $\begin{array}{l}-2,02 * * * \\
(0,000)\end{array}$ & $\begin{array}{l}-2,29 \\
(0,150)\end{array}$ & $\begin{array}{l}-2,18^{* *} \\
(0,007)\end{array}$ & $\begin{array}{l}-2,85^{*} \\
(0,011)\end{array}$ & $\begin{array}{l}-2,99 * * \\
(0,002)\end{array}$ & $\begin{array}{l}-1,03 \\
(0,170)\end{array}$ & $\begin{array}{l}-0,54 \\
(0,642)\end{array}$ & $\begin{array}{l}-3,20^{*} \\
(0,011)\end{array}$ \\
\hline$\beta_{c}^{\text {WORK } I V-M C O}$ & $\begin{array}{l}0,58 \\
(0,120)\end{array}$ & $\begin{array}{l}-2,66 \\
(0,109)\end{array}$ & $\begin{array}{l}0,92^{* *} \\
(0,007)\end{array}$ & $\begin{array}{l}0,38 \\
(0,680)\end{array}$ & $\begin{array}{l}0,68 \\
(0,639)\end{array}$ & $\begin{array}{l}0,20 \\
(0,830)\end{array}$ & $\begin{array}{l}1,72^{*} \\
(0,048)\end{array}$ & $\begin{array}{l}-0,03 \\
(0,935)\end{array}$ & & $\begin{array}{l}0,10 \\
(0,917)\end{array}$ \\
\hline \multirow[t]{3}{*}{$\mathrm{N}$} & 8472 & 6364 & 10327 & 2387 & 5925 & 8614 & 7177 & 8154 & 7038 & 2966 \\
\hline & Irlande & Israël & Italie & Luxembourg & Pologne & Portugal & Slovénie & Suède & Suisse & Tchéquie \\
\hline & \multicolumn{10}{|c|}{ Modèle 1: indicateur d'altération de la santé et indicateur d'altération de la cognition (MCO) } \\
\hline$\beta_{h}^{\text {WORK }} M C O$ & $\begin{array}{l}-5,80^{* *} \\
(0,002)\end{array}$ & $\begin{array}{l}-4,96 * * * \\
(0,000)\end{array}$ & $\begin{array}{l}-3,22 * * * \\
(0,000)\end{array}$ & $\begin{array}{l}-3,20^{*} \\
(0,015)\end{array}$ & $\begin{array}{l}-8,60^{* * *} \\
(0,000)\end{array}$ & $\begin{array}{l}-1,60 \\
(0,246)\end{array}$ & $\begin{array}{l}-5,97^{* * *} \\
(0,000)\end{array}$ & $\begin{array}{l}-6,78^{* * *} \\
(0,000)\end{array}$ & $\begin{array}{l}-3,75^{* * *} \\
(0,000)\end{array}$ & $\begin{array}{l}-8,32^{* * *} \\
(0,000)\end{array}$ \\
\hline \multirow[t]{2}{*}{$\beta_{c}^{\text {WORK }} M C O$} & $\begin{array}{l}-3,44 \\
(0,138)\end{array}$ & $\begin{array}{l}-2,09 \\
(0,079)\end{array}$ & $\begin{array}{l}-3,96 * * * \\
(0,000)\end{array}$ & $\begin{array}{l}-0,35 \\
(0,784)\end{array}$ & $\begin{array}{l}-0,66 \\
(0,549)\end{array}$ & $\begin{array}{l}0,10 \\
(0,951)\end{array}$ & $\begin{array}{l}-2,77^{* *} \\
(0,005)\end{array}$ & $\begin{array}{l}-0,73 \\
(0,396)\end{array}$ & $\begin{array}{l}-2,41^{* *} \\
(0,007)\end{array}$ & $\begin{array}{l}-2,49 * * \\
(0,001)\end{array}$ \\
\hline & \multicolumn{10}{|c|}{$\begin{array}{l}\text { Modèle 2: indicateur d'altération de la santé et indicateur d'altération de la cognition } \\
\text { (modèle à variables instrumentales) }\end{array}$} \\
\hline$\beta_{h}^{\text {WORK } I V}$ & $\begin{array}{l}-6,76^{*} \\
(0,019)\end{array}$ & $\begin{array}{l}-9,00^{* * *} \\
(0,000)\end{array}$ & $\begin{array}{l}-5,47^{* * *} \\
(0,000)\end{array}$ & $\begin{array}{l}-6,26 * \\
(0,026)\end{array}$ & $\begin{array}{l}-11,72^{* * *} \\
(0,000)\end{array}$ & $\begin{array}{l}-6,23^{* * *} \\
(0,000)\end{array}$ & $\begin{array}{l}-8,95^{* * *} \\
(0,000)\end{array}$ & $\begin{array}{l}-8,95^{* * *} \\
(0,000)\end{array}$ & $\begin{array}{l}-4,46^{* *} \\
(0,001)\end{array}$ & $\begin{array}{l}-12,22 * * * \\
(0,000)\end{array}$ \\
\hline \multirow[t]{2}{*}{$\beta_{c}^{\text {WORK } I V}$} & $\begin{array}{l}-2,57 \\
(0,319)\end{array}$ & $\begin{array}{l}-1,26 \\
(0,414)\end{array}$ & $\begin{array}{l}-3,99 * * * \\
(0,000)\end{array}$ & $\begin{array}{l}0,65 \\
(0,715)\end{array}$ & $\begin{array}{l}-0,75 \\
(0,548)\end{array}$ & $\begin{array}{l}0,24 \\
(0,912)\end{array}$ & $\begin{array}{l}-1,56 \\
(0,241)\end{array}$ & $\begin{array}{l}0,50 \\
(0,605)\end{array}$ & $\begin{array}{l}-2,10^{*} \\
(0,020)\end{array}$ & $\begin{array}{l}0,03 \\
(0,984)\end{array}$ \\
\hline & \multicolumn{10}{|c|}{ Comparaison des modèles 1 et 2} \\
\hline$\beta_{h}^{\text {WORK } I V-M C O}$ & $\begin{array}{l}-0,97 \\
(0,646)\end{array}$ & $\begin{array}{l}-4,04^{* * *} \\
(0,001)\end{array}$ & $\begin{array}{l}-2,25^{*} \\
(0,040)\end{array}$ & $\begin{array}{l}-3,06 \\
(0,171)\end{array}$ & $\begin{array}{l}-3,13^{* *} \\
(0,006)\end{array}$ & $\begin{array}{l}-4,63^{* *} \\
(0,005)\end{array}$ & $\begin{array}{l}-2,98^{* *} \\
(0,007)\end{array}$ & $\begin{array}{l}-2,17^{*} \\
(0,041)\end{array}$ & $\begin{array}{l}-0,71 \\
(0,498)\end{array}$ & $\begin{array}{l}-3,89 * * * \\
(0,000)\end{array}$ \\
\hline$\beta_{c}^{\text {WORK } I V-M C O}$ & $\begin{array}{l}0,87 \\
(0,670)\end{array}$ & $\begin{array}{l}0,82 \\
(0,352)\end{array}$ & $\begin{array}{l}-0,03 \\
(0,950)\end{array}$ & $\begin{array}{l}1,00 \\
(0,389)\end{array}$ & $\begin{array}{l}-0,09 \\
(0,883)\end{array}$ & $\begin{array}{l}0,14 \\
(0,919)\end{array}$ & $\begin{array}{l}1,20 \\
(0,174)\end{array}$ & $\begin{array}{l}1,22 * \\
(0,040)\end{array}$ & $\begin{array}{l}0,31 \\
(0,379)\end{array}$ & $\begin{array}{l}2,52 \\
(0,056)\end{array}$ \\
\hline $\mathrm{N}$ & 991 & 3845 & 8563 & 2004 & 2905 & 2023 & 5100 & 6866 & 4558 & 8134 \\
\hline
\end{tabular}

Note: Les symboles $*, * *$ et $* * *$ correspondent respectivement aux seuils de significativité statistique de 10 pour cent, 5 pour cent et 1 pour cent. Les valeurs $p$ sont indiquées entre parenthèses. Les erreurs type sous-jacentes ont été estimées au moyen de la méthode bootstrap (100 itérations). Le travail total (WORK) résulte de la combinaison du nombre d'heures travaillées (HOURS) et de l'emploi (EMPL). L'indicateur de santé subjectif/physique a été instrumenté par les variables objectives figurant dans le tableau 3. Diminution prédite par la dégradation de l'état de santé et/ou de la cognition ( 0 = niveau parmi les personnes de 50 à 54 ans).

Source: Calculs réalisés par l'auteur sur la base des données issues de SHARE (2004-2017). 
Tableau A3. Validité des items de santé objectifs en tant qu'instruments de l'indicateur de santé objectif

\begin{tabular}{lcc:cc}
\hline & $\begin{array}{l}\text { Statistique F de } \\
\text { Cragg-Donald Wald }\end{array}$ & $\begin{array}{l}\text { Statistique F de } \\
\text { Kleibergen-Paap } \\
\text { rk Wald }\end{array}$ & $\begin{array}{l}\text { Statistique LM de } \\
\text { Cragg-Donald Wald }\end{array}$ & $\begin{array}{l}\text { Statistique LM de } \\
\text { Cragg-Donald Wald } \\
\text { (valeur } p \text { ) }\end{array}$ \\
\hline Allemagne & 97,95 & 93,0086 & 414,24 & 0,0000 \\
Autriche & 58,24 & 65,4328 & 213,50 & 0,0000 \\
Belgique & 168,70 & 161,9989 & 483,90 & 0,0000 \\
Croatie & 30,00 & 70,2251 & 128,47 & 0,0000 \\
Danemark & 99,80 & 97,2003 & 328,62 & 0,0000 \\
Espagne & 93,70 & 81,0782 & 370,97 & 0,0000 \\
Estonie & 89,56 & 189,0850 & 340,82 & 0,0000 \\
France & 113,21 & 103,6121 & 401,69 & 0,0000 \\
Grèce & 82,54 & 70,1884 & 325,32 & 0,0000 \\
Hongrie & 28,63 & 51,4255 & 103,54 & 0,0000 \\
Irlande & 18,43 & 102,7409 & 35,75 & 0,0011 \\
Israël & 43,39 & 57,4291 & 163,60 & 0,0000 \\
Italie & 72,38 & 64,7244 & 344,10 & 0,0000 \\
Luxembourg & 13,03 & 40,7144 & 64,67 & 0,0000 \\
Pologne & 49,46 & 123,2353 & 198,35 & 0,0000 \\
Portugal & 17,07 & 19,0707 & 72,35 & 0,0000 \\
Slovénie & 40,00 & 40,8839 & 147,94 & 0,0000 \\
Suède & 38,31 & 41,0943 & 147,58 & 0,0000 \\
Suisse & 49,99 & 51,7269 & 165,68 & 0,0000 \\
Tchéquie & 69,22 & 186,2675 & 300,69 & 0,0000 \\
Note: & & & &
\end{tabular}

Note: Les tests de Cragg-Donald/Kleibergen-Paap Wald sont des tests d'identification faible. Ils constituent en substance un test $\mathrm{F}$ (ils testent la significativité des régresseurs de la première étape en vérifiant s'ils sont conjointement égaux à zéro) et prennent ainsi en compte la présence d'une hétéroscédasticité. Le test de sousidentification (Cragg-Donald Wald LM) est le test du multiplicateur de Lagrange (LM) permettant de déterminer si l'équation est identifiée, c'est-à-dire si les instruments exclus sont «pertinents», autrement dit corrélés avec les régresseurs endogènes. Ce test est en substance le test de rang d'une matrice: si l'on accepte l'hypothèse nulle de sous-identification de l'équation, la matrice de coefficients de forme réduite sur les instruments exclus de $L 1$ occupe le rang $=K 1-1$, où $K 1=$ nombre de régresseurs endogènes. Sous l'hypothèse nulle, la statistique est distribuée selon une loi de chi-carré avec des degrés de liberté égaux à ( $L 1-K 1+1)$. Un rejet de l'hypothèse nulle signifie que la matrice a un rang de colonne complet, c'est-à-dire que le modèle est identifié.

Source: Calculs réalisés par l'auteur sur la base des données issues de SHARE (2004-2017). 
Tableau A4. Résultats des première et deuxième étapes: impact de l'altération de l'état de santé/de la cognition sur le travail total (WORK) parmi les répondants âgés de 50 à 54 ans et comparaison entre l'impact de l'altération de la santé seulement et l'impact de l'altération de la santé et de la cognition estimés par le modèle à variables instrumentales

\begin{tabular}{|c|c|c|c|c|c|c|c|c|c|c|}
\hline & Allemagne & Autriche & Belgique & Croatie & Danemark & Espagne & Estonie & France & Grèce & Hongrie \\
\hline & \multicolumn{10}{|c|}{ A. Modèle 1 - variables instrumentales - Indicateur d'altération de la santé seulement } \\
\hline$\beta_{h}^{\text {WORK }}$ & $\begin{array}{l}-8,16^{* * *} \\
(0,000)\end{array}$ & $\begin{array}{l}-8,97^{* * *} \\
(0,000)\end{array}$ & $\begin{array}{l}-7,95^{* * * *} \\
(0,000)\end{array}$ & $\begin{array}{l}-5,49 * * \\
(0,010)\end{array}$ & $\begin{array}{l}-8,80 * * * \\
(0,000)\end{array}$ & $\begin{array}{l}-8,90 * * * \\
(0,000)\end{array}$ & $\begin{array}{l}-7,87^{* * * *} \\
(0,000)\end{array}$ & $\begin{array}{l}-6,58^{* * *} \\
(0,000)\end{array}$ & $\begin{array}{l}-3,83^{*} \\
(0,011)\end{array}$ & $\begin{array}{l}-10,78^{* * * *} \\
(0,000)\end{array}$ \\
\hline \multirow[t]{2}{*}{$\begin{array}{l}\text { Diminution } \\
{[\mathrm{A}]}\end{array}$} & $\begin{array}{l}-3,89 * * * \\
(0,000)\end{array}$ & $\begin{array}{l}-6,56 \\
(0,089)\end{array}$ & $\begin{array}{l}-6,11^{* * * *} \\
(0,000)\end{array}$ & $\begin{array}{l}-7,67^{* * *} \\
(0,000)\end{array}$ & $\begin{array}{l}-6,91 \\
(0,110)\end{array}$ & $\begin{array}{l}-8,62^{* * *} \\
(0,000)\end{array}$ & $\begin{array}{l}-7,03^{* * *} \\
(0,000)\end{array}$ & $\begin{array}{l}-6,76^{* * *} \\
(0,000)\end{array}$ & $\begin{array}{l}-5,28^{* * *} \\
(0,000)\end{array}$ & $\begin{array}{l}-18,13^{* * * *} \\
(0,000)\end{array}$ \\
\hline & \multicolumn{10}{|c|}{ B. Modèle 2 - variables instrumentales - Indicateur d'altération de la santé et de la cognition } \\
\hline$\beta_{h}^{\text {WORK }}$ & $\begin{array}{l}-6,59 * * * \\
(0,000)\end{array}$ & $\begin{array}{l}-7,62^{* * *} \\
(0,000)\end{array}$ & $\begin{array}{l}-6,85^{* * * *} \\
(0,000)\end{array}$ & $\begin{array}{l}-7,37^{* * *} \\
(0,000)\end{array}$ & $\begin{array}{l}-8,27^{* * *} \\
(0,000)\end{array}$ & $\begin{array}{l}-6,52^{* * *} \\
(0,000)\end{array}$ & $\begin{array}{l}-7,23^{* * *} \\
(0,000)\end{array}$ & $\begin{array}{l}-5,90^{* * *} \\
(0,000)\end{array}$ & $\begin{array}{l}-5,63^{* * *} \\
(0,000)\end{array}$ & $\begin{array}{l}-9,18^{* * *} \\
(0,000)\end{array}$ \\
\hline$\beta_{c}^{\text {WORK }}$ & $\begin{array}{l}-1,99 * * \\
(0,002)\end{array}$ & $\begin{array}{l}-0,92 \\
(0,262)\end{array}$ & $\begin{array}{l}-3,35^{* * * *} \\
(0,000)\end{array}$ & $\begin{array}{l}-0,41 \\
(0,809)\end{array}$ & $\begin{array}{l}-2,20^{* * * *} \\
(0,000)\end{array}$ & $\begin{array}{l}-5,76^{* * * *} \\
(0,000)\end{array}$ & $\begin{array}{l}-2,61^{* * * *} \\
(0,000)\end{array}$ & $\begin{array}{l}-3,99 * * * \\
(0,000)\end{array}$ & $\begin{array}{l}-1,15 \\
(0,253)\end{array}$ & $\begin{array}{l}-3,01 * \\
(0,022)\end{array}$ \\
\hline \multirow[t]{2}{*}{$\begin{array}{l}\text { Diminution } \\
\text { [B] }\end{array}$} & $\begin{array}{l}-4,03^{* * * *} \\
(0,000)\end{array}$ & $\begin{array}{l}-2,86^{* * *} \\
(0,000)\end{array}$ & $\begin{array}{l}-4,30 * * * \\
(0,000)\end{array}$ & $\begin{array}{l}-4,12^{* * *} \\
(0,000)\end{array}$ & $\begin{array}{l}-3,93^{* * *} \\
(0,000)\end{array}$ & $\begin{array}{l}-7,32^{* * *} \\
(0,000)\end{array}$ & $\begin{array}{l}-5,78^{* * * *} \\
(0,000)\end{array}$ & $\begin{array}{l}-4,24^{* * *} \\
(0,000)\end{array}$ & $\begin{array}{l}-4,23 * * * \\
(0,000)\end{array}$ & $\begin{array}{l}-7,56 * * * \\
(0,000)\end{array}$ \\
\hline & \multicolumn{10}{|c|}{ C. Comparaison des modèles 1 et 2} \\
\hline $\begin{array}{l}\text { Diminution } \\
{[\mathrm{A}]-[\mathrm{B}]}\end{array}$ & $\begin{array}{l}-0,14 \\
(0,891)\end{array}$ & $\begin{array}{l}3,71 \\
(0,337)\end{array}$ & $\begin{array}{l}1,81^{* *} \\
(0,002)\end{array}$ & $\begin{array}{l}3,55^{*} \\
(0,012)\end{array}$ & $\begin{array}{l}2,98 \\
(0,489)\end{array}$ & $\begin{array}{l}1,30 \\
(0,583)\end{array}$ & $\begin{array}{l}1,25 \\
(0,113)\end{array}$ & $\begin{array}{l}2,52 * \\
(0,037)\end{array}$ & $\begin{array}{l}1,05 \\
(0,376)\end{array}$ & $\begin{array}{l}10,56^{* * *} \\
(0,000)\end{array}$ \\
\hline \multirow[t]{2}{*}{$\mathrm{N}$} & 8472 & 6364 & 10327 & 2387 & 5925 & 8614 & 7177 & 8154 & 7038 & 2966 \\
\hline & Irlande & Israël & Italie & Luxembourg & Pologne & Portugal & Slovénie & Suède & Suisse & \\
\hline & \multicolumn{10}{|c|}{ A. Modèle 1 - variables instrumentales - Indicateur d'altération de la santé seulement } \\
\hline$\beta_{h}^{\text {WORK }}$ & $\begin{array}{l}-7,18^{*} \\
(0,011)\end{array}$ & $\begin{array}{l}-9,44^{* * *} \\
(0,000)\end{array}$ & $\begin{array}{l}-4,84^{* * * *} \\
(0,000)\end{array}$ & $\begin{array}{l}-4,87 \\
(0,081)\end{array}$ & $\begin{array}{l}-6,25^{* * *} \\
(0,000)\end{array}$ & $\begin{array}{l}-5,34^{* *} \\
(0,002)\end{array}$ & $\begin{array}{l}-8,11^{* * *} \\
(0,000)\end{array}$ & $\begin{array}{l}-8,75^{* * * *} \\
(0,000)\end{array}$ & $\begin{array}{l}-4,70 * * * \\
(0,001)\end{array}$ & $\begin{array}{l}-10,22^{* * * *} \\
(0,000)\end{array}$ \\
\hline \multirow[t]{2}{*}{$\begin{array}{l}\text { Diminution } \\
{[\mathrm{A}]}\end{array}$} & $\begin{array}{l}-4,12 \\
(0,137)\end{array}$ & $\begin{array}{l}-12,08^{* * *} \\
(0,000)\end{array}$ & $\begin{array}{l}-6,10^{*} \\
(0,040)\end{array}$ & $\begin{array}{l}-6,40^{*} \\
(0,012)\end{array}$ & $\begin{array}{l}-6,07^{*} \\
(0,014)\end{array}$ & $\begin{array}{l}3,85 \\
(0,177)\end{array}$ & $\begin{array}{l}-9,05^{* * *} \\
(0,000)\end{array}$ & $\begin{array}{l}-10,88^{* * *} \\
(0,000)\end{array}$ & $\begin{array}{l}-3,61 \\
(0,552)\end{array}$ & $\begin{array}{l}-12,59 \\
(0,110)\end{array}$ \\
\hline & \multicolumn{10}{|c|}{ B. Modèle 2 - variables instrumentales - Indicateur d'altération de la santé et de la cognition } \\
\hline$\beta_{h}^{\text {WORK }}$ & $\begin{array}{l}-6,99 * * * \\
(0,000)\end{array}$ & $\begin{array}{l}-5,45^{* * * *} \\
(0,000)\end{array}$ & $\begin{array}{l}-4,03^{* * *} \\
(0,000)\end{array}$ & $\begin{array}{l}-5,88^{* * *} \\
(0,000)\end{array}$ & $\begin{array}{l}-8,92^{* * * *} \\
(0,000)\end{array}$ & $\begin{array}{l}-2,15 \\
(0,116)\end{array}$ & $\begin{array}{l}-6,97^{* * *} \\
(0,000)\end{array}$ & $\begin{array}{l}-7,92^{* * *} \\
(0,000)\end{array}$ & $\begin{array}{l}-5,34^{* * *} \\
(0,000)\end{array}$ & $\begin{array}{l}-9,14^{* * *} \\
(0,000)\end{array}$ \\
\hline$\beta_{c}^{\text {WORK }}$ & $\begin{array}{l}-2,04 \\
(0,396)\end{array}$ & $\begin{array}{l}-4,42^{* * * *} \\
(0,000)\end{array}$ & $\begin{array}{l}-6,06^{* * * *} \\
(0,000)\end{array}$ & $\begin{array}{l}-0,31 \\
(0,803)\end{array}$ & $\begin{array}{l}-0,85 \\
(0,415)\end{array}$ & $\begin{array}{l}-0,86 \\
(0,511)\end{array}$ & $\begin{array}{l}-4,87^{* * *} \\
(0,000)\end{array}$ & $\begin{array}{l}-1,47 \\
(0,081)\end{array}$ & $\begin{array}{l}-1,51 \\
(0,090)\end{array}$ & $\begin{array}{l}-3,18^{* * * *} \\
(0,000)\end{array}$ \\
\hline \multirow[t]{2}{*}{$\begin{array}{l}\text { Diminution } \\
{[\mathrm{B}]}\end{array}$} & $\begin{array}{l}-3,97^{*} \\
(0,010)\end{array}$ & $\begin{array}{l}-8,52^{* * * *} \\
(0,000)\end{array}$ & $\begin{array}{l}-6,24^{* * * *} \\
(0,000)\end{array}$ & $\begin{array}{l}-2,90 * * \\
(0,004)\end{array}$ & $\begin{array}{l}-7,76^{* * *} \\
(0,000)\end{array}$ & $\begin{array}{l}-1,49^{*} \\
(0,040)\end{array}$ & $\begin{array}{l}-6,62^{* * *} \\
(0,000)\end{array}$ & $\begin{array}{l}-2,62^{* * * *} \\
(0,000)\end{array}$ & $\begin{array}{l}-2,07^{* * *} \\
(0,000)\end{array}$ & $\begin{array}{l}-7,08^{* * * *} \\
(0,000)\end{array}$ \\
\hline & \multicolumn{10}{|c|}{ C. Comparaison des modèles 1 et 2} \\
\hline $\begin{array}{l}\text { Diminution } \\
{[\mathrm{A}]-[\mathrm{B}]}\end{array}$ & $\begin{array}{l}0,15 \\
(0,955)\end{array}$ & $\begin{array}{l}3,55 \\
(0,127)\end{array}$ & $\begin{array}{l}-0,14 \\
(0,962)\end{array}$ & $\begin{array}{l}3,50 \\
(0,137)\end{array}$ & $\begin{array}{l}-1,69 \\
(0,476)\end{array}$ & $\begin{array}{l}-5,34 \\
(0,059)\end{array}$ & $\begin{array}{l}2,43^{*} \\
(0,044)\end{array}$ & $\begin{array}{l}8,26 * * \\
(0,001)\end{array}$ & $\begin{array}{l}1,54 \\
(0,799)\end{array}$ & $\begin{array}{l}5,51 \\
(0,485)\end{array}$ \\
\hline $\mathrm{N}$ & 991 & 3845 & 8563 & 2004 & 2905 & 2023 & 5100 & 6866 & 4558 & 8134 \\
\hline
\end{tabular}

Note: Les symboles $*, * *$ et $* * *$ correspondent respectivement aux seuils de significativité statistique de 10 pour cent, 5 pour cent et 1 pour cent. Les valeurs $p$ sont indiquées entre parenthèses. Les erreurs type sous-jacentes ont été estimées au moyen de la méthode bootstrap (100 itérations). Le travail total (WORK) résulte de la combinaison du nombre d'heures travaillées (HOURS) et de l'emploi (EMPL). L'indicateur de santé subjectif/physique a été instrumenté par les variables objectives figurant dans le tableau 3. Diminution prédite par la dégradation de l'état de santé et/ou de la cognition ( 0 = niveau parmi les personnes de 50 à 54 ans).

Source: Calculs réalisés par l'auteur sur la base des données issues de SHARE (2004-2017). 